\title{
Extraordinary Transmission Filtering Structures based on Plasmonic Metamaterials
}

\section{Doctoral Thesis}

Master in Communication Technology, Systems and Networks

\section{Rubén Ortuño Molinero}

Supervisor: Dr. Alejandro José Martínez Abiétar

Thesis submitted for the degree of

Doctor of Philosophy in Telecommunications Engineering

Valencia, October 2011 
Universidad Politécnica de Valencia

I.U.I. de Tecnología Nanofotónica - Nanophotonics Technology Center Camino de Vera, s/n. Building 8F, 46022, Valencia, Spain

Phone +34 963879768, Fax +34 963877827

ruormo@ntc.upv.es

www.ntc.upv.es 




\section{Acknowledgements}

First of all, I wish to express my gratitude to Prof. Javier Martí and my supervisor Prof. Alejandro Martínez for giving me the opportunity to pursue research at Nanophotonics Technology Center. Thanks to their constant advice, encouragement and dedication, I could do my Doctoral Thesis in this fascinating world of Metamaterials. Their high demanding and proficiency have transferred me the importance of being rigorous and the enthusiasm to achieve great goals. Values that I hope to preserve all of my life.

I would also like to thank my fellows Carlos García Meca and José Francisco Rodríguez Fortuño for the discussions we had about physics and the good times spent together. Thanks to all my colleagues at NTC, particularly to José Vicente Galán, Antoine Brimont, Guillermo Villanueva, Jesús Palací, Mercé LLopis, José María Escalante, Javier García, María Lorente, Irene Alepuz and Begoña Tomás, for their companionship and friendship. Through this work I had the opportunity to meet many other researchers, among whom I would like to specifically mention Miguel Navarro for his friendship and enjoyable talks both at work and away.

Finally, I would like to acknowledge my family, girlfriend and friends from school and university. I especially like to dedicate this work to my parents, without whose unconditional support and understanding this thesis would not have been possible. 


\section{Resumen}

Esta tesis trata sobre el fascinante fenómeno de la transmisión extraordinaria a través de láminas metálicas nonoestructuradas periódicamente con aperturas al corte. Un efecto relacionado con la excitación de un tipo de ondas superficiales como son los plasmones de superficie. Además, en aquellas estructuras formadas por el apilamiento de dos o más láminas metálicas se consiguen nuevas funcionalidades, como magnetismo artificial que da lugar a resonancias magnéticas y por tanto la posibilidad de obtener un índice de refracción negativo. Mediante un estudio teórico y numérico se ha comprobado que este tipo de respuesta magnética efectiva se debe a la excitación de resonancias plasmónicas internas en la estructura. Obteniéndose, bajo incidencia normal, un índice de refracción efectivo negativo en la dirección de propagación en el caso de que dichas resonancias se produzcan en zonas del espectro donde se obtenga una permitividad negativa, conectando el mundo de la plasmónica con el de los metamateriales.

Uno de los principales objetivos en el diseño de metamateriales es obtener un índice de refracción negativo en un gran ancho de banda. Sin embargo, este objetivo suele ser complicado de conseguir al basar los diseños en fenómenos resonantes. Es por ello que en esta tesis se ha propuesto un diseño basado en el apilamiento de estructuras fishnet con diferentes grosores de dieléctrico para conseguir aumentar el ancho de banda en el cual se consigue un índice negativo. Básicamente, la obtención de tal efecto se basa en la excitación de resonancias plasmónicas a distintas frecuencias al estar formada la celda unidad por diferentes grosores de dieléctrico. La hibridación que se produce entre dichas resonancias permite aumentar el ancho de banda con índice negativo.

Aunque la transmisión extraordinaria esta principalmente relacionada con la excitación de plasmones de superficie, los resultados mostrados en la tesis demuestran que para el caso de láminas metálicas rodeadas por dieléctricos también se consigue transmisión extraordinaria debido a la adaptación de la luz incidente a los modos soportados por los medios dieléctricos siempre y cuando el metal se encuentre estructurado periódicamente. Estos resultados demuestran el importante papel de la periodicidad en la aparición del fenómeno de la transmisión extraordinaria no solo mediante la excitación de plasmones de superficie sino también de los modos dieléctricos. 
Finalmente, se muestran resultados experimentales de transmisión a través de varias estructuras metálicas monocapa nanoestructuradas, fabricadas variando los parámetros de diseño, tales como medios dieléctricos, tamaño de aperturas y periodicidad, para estudiar su influencia en la respuesta espectral. Concretamente sobre la frecuencia de resonancia, factor de calidad y niveles de transmisión.

Todas estas investigaciones se realizaron con la intención de poder usar dichas estructuras como componentes ópticos ultracompactos en futuras aplicaciones basadas en sistemas nano-ópticos. Aplicaciones, entre otras, como detección, focalización de luz, o como las presentadas en la tesis de filtrado y switching óptico. 


\section{Resum}

Esta tesi tracta sobre el fascinant fenomen de la transmissió extraordinària a través de làmines metàl · liques nonoestructurades periòdicament amb obertures al tall. Un efecte relacionat amb l'excitació d'un tipus d'ones superficials com són els plasmons de superfície. A més, en aquelles estructures formades per l'apilament de dos o més làmines metàl liques s'aconseguixen noves funcionalitats, com a magnetisme artificial que dóna lloc a ressonàncies magnètiques i per tant la possibilitat d'obtindre un índex de refracció negatiu. Per mitjà d'un estudi teòric i numèric s'ha comprovat que este tipus de resposta magnètica efectiva es deu a l'excitació de ressonàncies plasmóniques internes en l'estructura. Obtenint-se, davall incidència normal, un índex de refracció efectiu negatiu en la direcció de propagació en el cas que dites ressonàncies es produïsquen en zones de l'espectre on s'obtinga una permitivitat negativa, connectant el món de la plasmónica amb el dels metamaterials.

Un dels principals objectius en el disseny de metamaterials és obtindre un índex de refracció negatiu en un gran ample de banda. No obstant, este objectiu sol ser complicat d'aconseguir al basar els dissenys en fenòmens ressonants. És per això que en esta tesi s'ha proposat un disseny basat en l'apilament d'estructures fishnet amb diferents grossors de dielèctric per a aconseguir augmentar l'ample de banda en el qual s'aconseguix un índex negatiu. Bàsicament, l'obtenció de tal efecte es basa en l'excitació de ressonàncies plasmóniques a distintes freqüències a l'estar formada la cel · la unitat per diferents grossors de dielèctric. L'hibridació que es produïx entre dites ressonàncies permet augmentar l'ample de banda amb índex negatiu.

Encara que la transmissió extraordinària esta principalment relacionada amb l'excitació de plasmons de superfície, els resultats mostrats en la tesi demostren que per al cas de làmines metàl · liques rodejades per dielèctrics també s'aconseguix transmissió extraordinària a causa de l'adaptació de la llum incident als modes suportats pels mitjans dielèctrics sempre que el metall es trobe estructurat periòdicament. Estos resultats demostren l'important paper de la periodicitat en l'aparició del fenomen de la transmissió extraordinària no sols per mitjà de l'excitació de plasmons de superfície sinó també dels modes dielèctrics. 
Finalment, es mostren resultats experimentals de transmissió a través de diverses estructures metàl - liques monocapa nanoestructurades, fabricades variant els paràmetres de disseny, tals com medis dielèctrics, tamany d'obertures i periodicitat, per a estudiar la seua influència en la resposta espectral. Concretament sobre la freqüència de ressonància, factor de qualitat i nivells de transmissió.

Totes estes investigacions es van realitzar amb la intenció de poder usar dites estructures com a components òptics ultracompactes en futures aplicacions basades en sistemes nano-óptics. Aplicacions, entre altres, com a detecció, focalització de llum, o com les presentades en la tesi de filtrat i switching òptic. 


\section{Abstract}

This thesis is about the intriguing phenomenon of extraordinary transmission through metallic sheets periodically nanostructured with subwavelength apertures. An effect related with the excitement of a type of surface waves such as surface plasmons. Furthermore, in structures formed by stacking two or more metallic sheets, new features appear, such as artificial magnetism which gives rise to magnetic resonances and therefore the possibility to get a negative refractive index. By means of theoretical and numerical study, this type of effective magnetic response has been proved to be due to the excitation of plasmonic resonances in the internal structure. Retrieving, under normal incidence, an effective negative refractive index in the direction of propagation in the case of that these resonances occur within frequency regions where negative permittivity is obtained, connecting the world of plasmonics with that of metamaterials.

One of the main goals in the design of metamaterials is to achieve a negative refractive index over a broad bandwidth. However, this objective is usually difficult to accomplish owing to most of the designs rely on resonant phenomena. That is why in this thesis, a design based on stacking fishnet structures with different dielectric thicknesses has been proposed to increase the bandwidth at which a negative index is achieved. Basically, obtaining this effect is based on the excitation of plasmonic resonances at different frequencies as the unit cell is made up of different dielectric thicknesses. The hybridization effect that occurs among these resonances permits negative index bandwidth to increase.

Although the extraordinary transmission is primarily related to the excitation of surface plasmons, the results shown in the thesis demonstrate that in the case of metallic sheets surrounded by dielectric extraordinary transmission is also achieved due to the coupling of the impinging light to the modes supported by the dielectric media as long as the metal is periodically patterned. These results demonstrate the important role of the periodicity in the onset of the extraordinary transmission phenomenon not only through the excitation of surface plasmon but also dielectric modes.

Finally, experimental transmission results are shown through several nanostructured monolayer metallic structures, fabricated varying design parameters such as dielectric media, aperture size and lattice, in order to study its 
influence on the spectral response. Specifically, on the resonant frequency, quality factor and transmission level.

All these investigations were made with the aim of using these structures as ultracompact optical components in future applications based on nano-optical systems. Applications such as, among others, detection, light focusing, or as the presented in the thesis of filtering and optical switching. 


\section{Table of Contents}

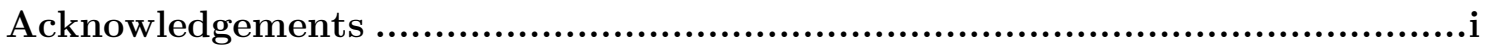

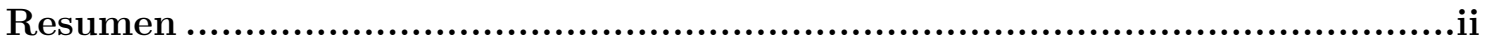

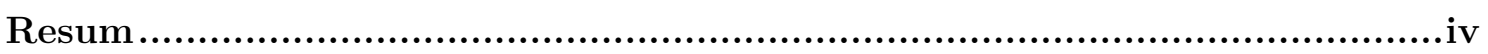

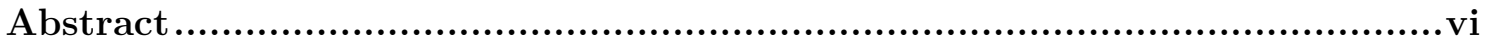

Plasmonic Metamaterials.............................................................. 1

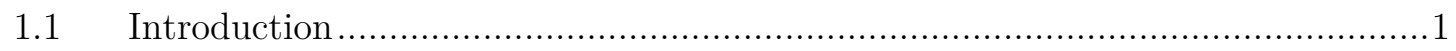

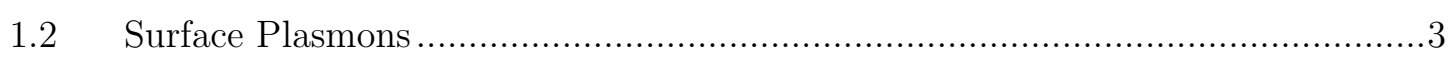

1.2.1. Surface plasmon on planar interfaces. …................................................. 5

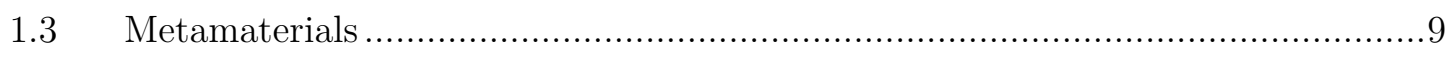

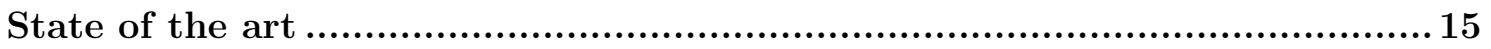

2.1 A new phenomenon: Extraordinary transmission …................................... 15

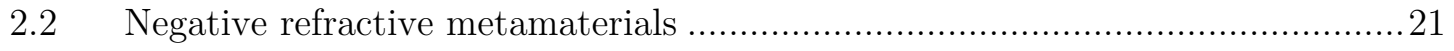

EOT and negative index in fishnet metamaterials ............................25

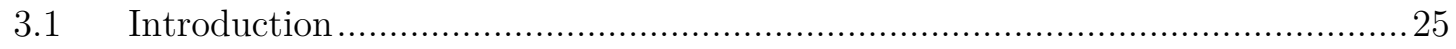

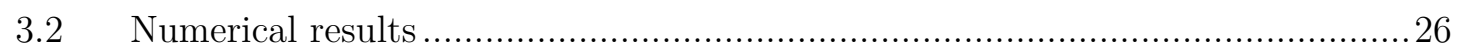

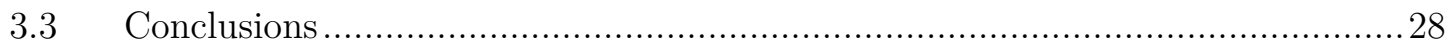

Enlarging the negative-index bandwidth by hybridized plasmon resonances. 31

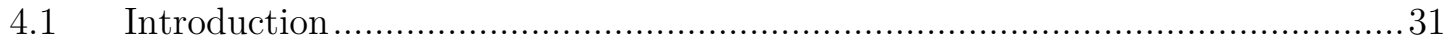

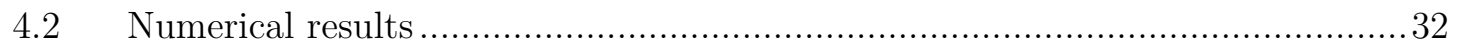

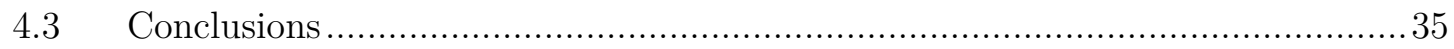

EOT from dielectric-guided modes .................................................. 37

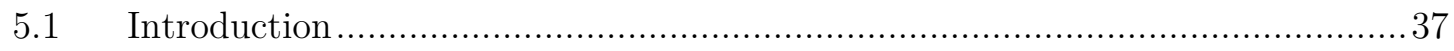

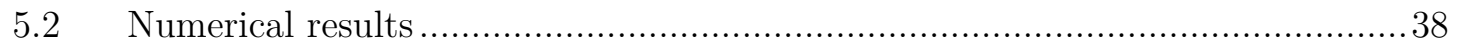

$5.3 \quad$ Ultrafast all-optical switching application ............................................... 40

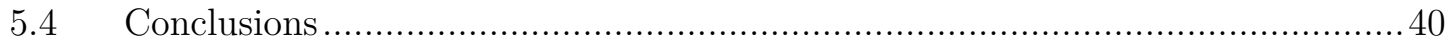

Implementation of EOT based filters at midinfrared range ...................... 41

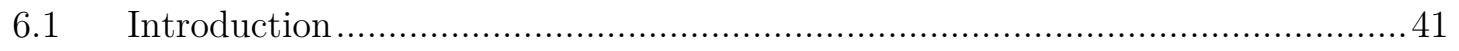

6.2 Fabrication and characterization processes................................................... 43

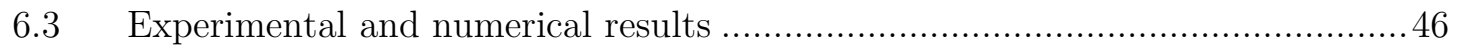




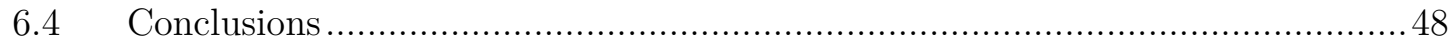

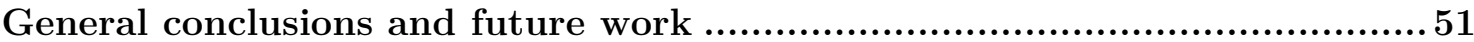

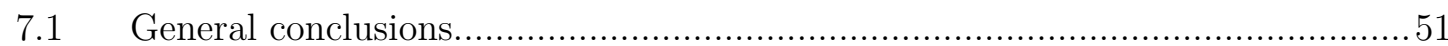

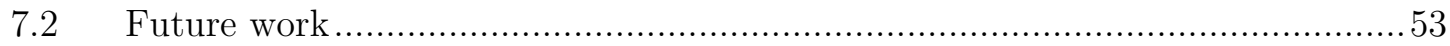

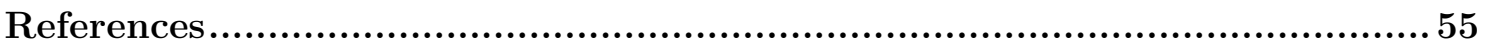

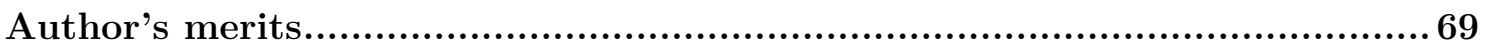

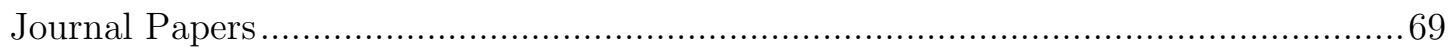

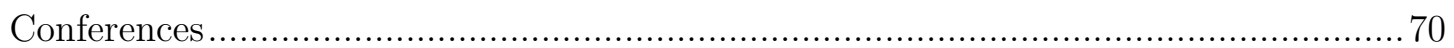





\section{Chapter 1}

\section{Plasmonic Metamaterials}

\subsection{Introduction}

In recent years, we have witnessed a flurry of activity in the fundamental research and development of surface plasmon based structures and devices, pushing the boundaries of the field of surface plasmon nanophotonics. Surface plasmons are collective charge oscillations that occur at the interface between conductors and dielectrics [RAE-86]. Their unique properties enable a wide range of practical applications, including light guiding and manipulation at the nanoscale, biodetection at the single molecule level, enhanced optical transmission through subwavelength apertures, and high resolution optical imaging below the diffraction limit [BAR-03]. These developments have led to the concept of plasmonics [BRO-99], which can be defined as the science and technology of metal-based optics and nanophotonics. Plasmonics explores how electromagnetic fields can be confined over dimensions on the order of or smaller than the wavelength.

A possible classification of nanophotonic structures can be done according to their spatial distribution (periodic or non-periodic) and to the material employed in their manufacturing (metal or dielectric), as shown in Fig. 1 along with the achieved functionalities. In this sense, plasmonics forms a major part of the field of nanophotonics, as it will cover the subset of periodical and nonperiodical metal-based nanophotonics structures. Indeed, the field of plasmonics represents an exciting new area for the application of surface plasmons, an area in 
which surface plasmon based circuits merge the fields of photonics and electronics at the nanoscale [OZB-06].

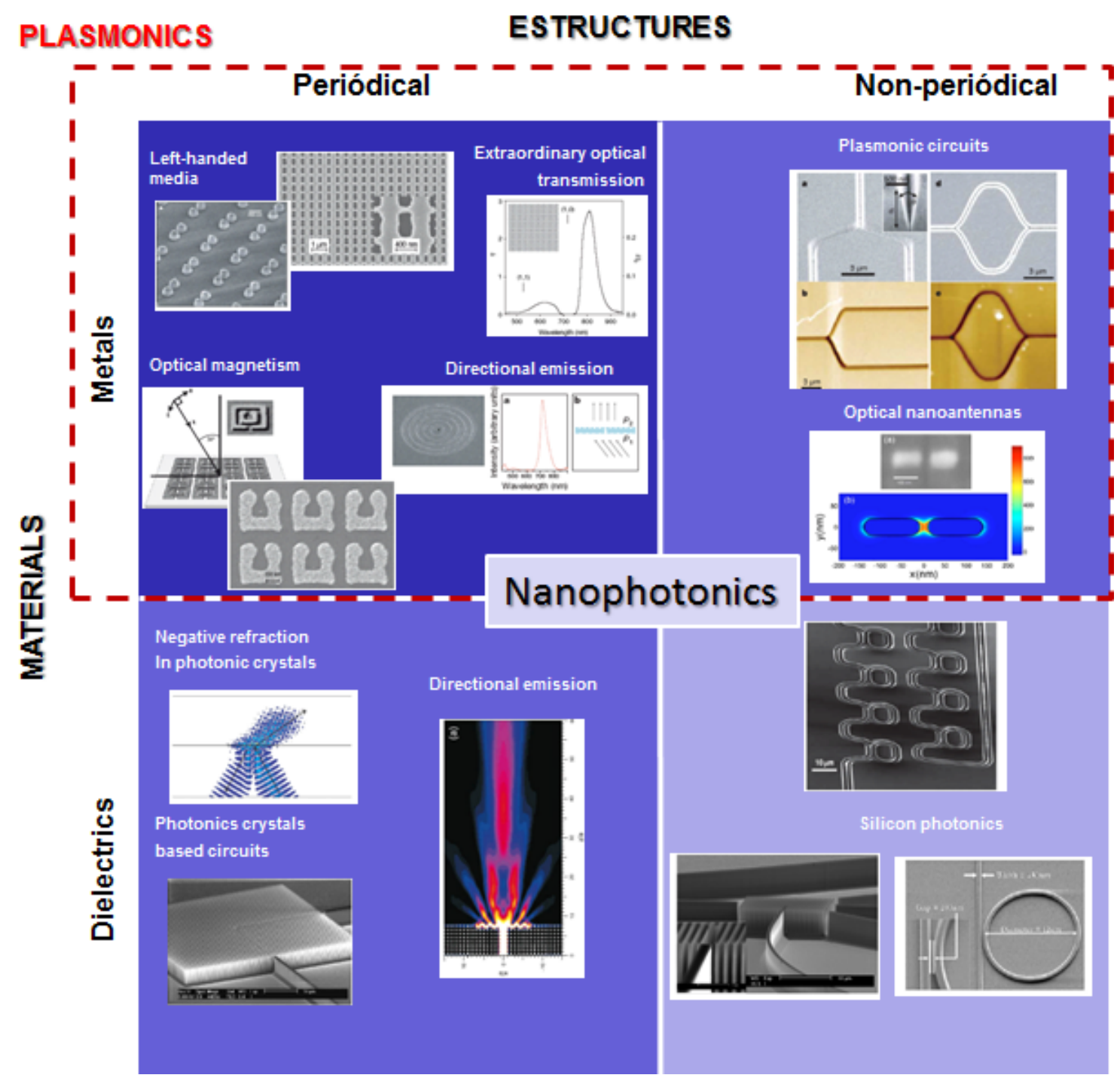

Figure 1. Classification of the nanophotonics structures according to their spatial distribution and constituent material.

Most recently, renewed interest in surface plasmons has come from recent advances in the investigation of the electromagnetic properties of metallic nanostructured materials, also referred to as plasmonic nanostructures, showing a plethora of amazing effects and fascinating phenomena [PEN-99], [BAR-03]: Specifically, the interest in surface plasmons sparked off in 1998 when Thomas Ebbesen and coworkers reported on the extraordinary optical transmission through subwavelength aperture arrays in optically thick metallic films [EBB-98]. On the other hand, the concern for a new kind of artificial materials started out with the seminal works of David Smith's group related to a negative refractive material [SMI-00] and Sir John Pendry that, based on the unusual 
electromagnetic property of negative refraction, suggested that a thin metallic film may act as a perfect lens [PEN-00]. Such artificial composites with unprecedented electromagnetic properties were termed metamaterials, meaning that they are something well beyond the natural materials. Metamaterials provide newly accessible electromagnetic properties arising from the subwavelength geometry of their constituent plasmonic nanostructures rather than from their chemical composition. The structural units of metamaterials can be tailored in shape and size, their composition and morphology can be artificially tuned, and inclusions can be designed and placed at desired locations to achieve new functionalities unattainable from natural materials.

In overall, the newfound ability to use plasmonic metamaterials has opened a myriad of exciting opportunities exhibiting fascinating optical properties never seen in the macro-world. Based on the exponential annual increase in the number of publications related to plasmonic metamaterials, it is clear that we are at the eve of a new revolution that will impact many fields of science and technology, including photonics, computation, biology, medicine, materials science, physics, and photovoltaics. Particularly, this Doctoral Thesis deals with plasmonic metamaterials and their implementation as ultra-compact optical nanostructures based on extraordinary transmission.

\subsection{Surface Plasmons}

Although the first scientific studies in which surface plasmons were observed date back to the beginning of the twentieth century, the unique optical properties of metal nanostructures were employed well before by artists to generate vibrant colors in glass artifacts and in the staining of church windows. One of the most famous examples is the Lycurgus cup dating back to the Byzantine Empire $\left(4^{\text {th }}\right.$ century AD), shown in Fig. 2. 


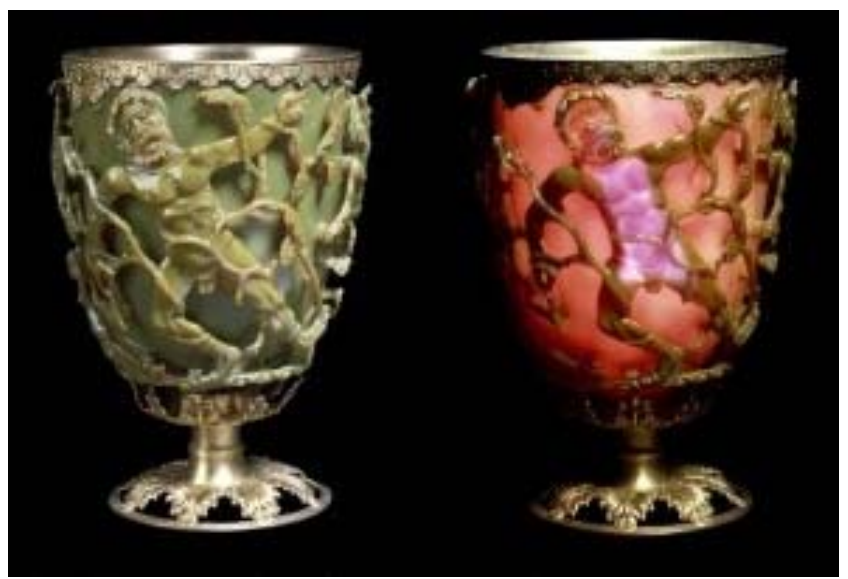

Figure 2. The Lycurgus cup. When viewed in reflected light it appears green. However, when a light is shone into the cup and transmitted through the glass, it appears red.

The mathematical description of these surface waves was established in the context of radio waves propagating along the surface of a conductor of finite conductivity [SOM-99], [ZEN-07]. In 1902 R.W. Wood observed anomalous intensity drops in spectra produced when visible light reflects at metallic gratings [WOO-02]. In 1956, in analogy to earlier work on plasma oscillations in gas discharges, D. Pines introduced the term "plasmons" to refer to the collective oscillations of free electrons in metals [PIN-56]. Coincidentally, in that same year R. Fano introduced the term "polariton" for the coupled oscillation of bound electrons and light inside transparent media [FAN-56]. Around that time, a study was published on loss phenomena associated with interactions taking place at metallic surfaces, in which it was shown that plasmon modes can exist near the surface of metals [RIT-57]. This study represents the first theoretical description of surface plasmons, which in 1968 were linked to the Wood's original observations, describing the anomalous behavior of metal gratings in terms of surface plasmon resonances excited on the gratings [RIT-68]. By that time, the excitation of surface plasmon with visible light using prism coupling had been achieved [OTT-68], [KRE-68], making experiments on surface plasmons easily accessible to many researchers. As the field continued to develop and the importance of the coupling between the oscillating electrons and the electromagnetic field became more apparent, S. Cunningham and coworkers introduced the term "surface plasmon polariton" (SPP) in 1974 [CUN-74].

All these discoveries set the stage for the current surge in surface plasmon nanophotonics, just at a time when crucial technological areas such as optical lithography and optical data storage are approaching fundamental physical limits. 
Several current technological challenges may be overcome by utilizing the unique properties of SPPs. Indeed, SPPs can serve as a basis for constructing nanoscale photonic circuits that will be able to carry optical signal and electric currents [BAR-03], as well as SPPs can also serve as a basis for the design, fabrication and characterization of subwavelength waveguide components [BOZ-06], [MAI-03], [KRE-04], [NOV-02]. Likewise, in the framework of plasmonics, modulators and switches have also been investigated [KRA-04], [KRA-05], [CHE-06], [PAU-09], [SHR-11]. Nowadays, SPPs have also a significant relevance to metamaterials as they occupy a central position both in the theoretical formulations and in establishing a physical picture.

\subsubsection{Surface plasmon on planar interfaces.}

Before embarking on the understanding of extraordinary optical transmission (EOT) in terms of SPPs, we firstly review the fundamentals of SPPs at a single flat interface and describe experimental techniques for their excitation.

SPPs are electromagnetic excitations propagating at the interface between a dielectric and a conductor, evanescently confined in the perpendicular direction. Thus, in order to investigate the physical properties of SPPs, we have to apply Maxwell's equations to the flat interface between a conductor and a dielectric. In the absence of external charge and current densities, assuming nonmagnetic media with dielectric responses without spatial dispersion $\varepsilon(\omega)=\varepsilon(\omega, \boldsymbol{r})$, and, without loss of generality, a harmonic time dependence of the electromagnetic field $\boldsymbol{E}(\boldsymbol{r}, t)=\boldsymbol{E}(\boldsymbol{r}) e^{-i \omega t}$, we arrive, after some straightforward manipulations of Maxwell's equations, at the central equation of electromagnetic wave theory, the Helmholtz equation,

$$
\nabla^{2} \boldsymbol{E}+k_{0}^{2} \varepsilon \boldsymbol{E}=0
$$

where $k_{0}=\frac{\omega}{c}$ is the wave vector of the propagating wave in vacuum. Practically, this equation has to be solved separately in regions of constant permittivity, and the obtained solutions have to be matched using appropriate boundary conditions. 


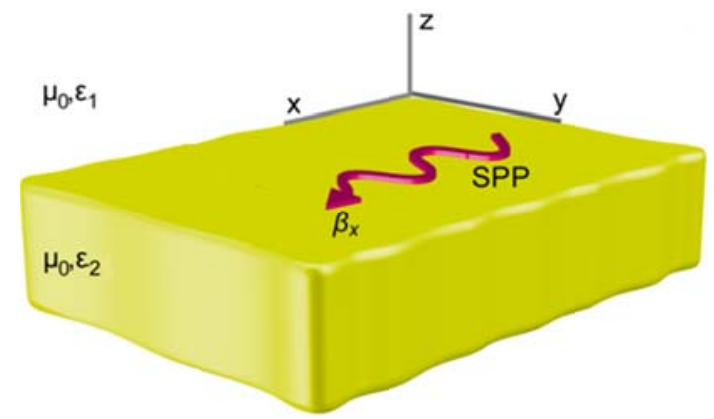

Figure 3. Geometry for SPPs propagation along the x-direction of a Cartesian coordinate system.

Afterwards, we define the propagation geometry, where we assume for simplicity a one-dimensional problem, i.e. an invariant geometry along the xyplane of a Cartesian coordinate system as depicted in Fig. 3. Specifically, the waves propagate along the $\mathrm{x}$-direction, and show no spatial variation in the perpendicular, in-plane y-direction. The plane $z=0$ coincides with the interface sustaining the propagating waves, which can now be described as $\boldsymbol{E}(\boldsymbol{r})=$ $\boldsymbol{E}(z) e^{i \beta_{x} x}$. The complex parameter $\beta_{x}$ is called the propagation constant of the travelling waves and corresponds to the component of the wave vector in the direction of propagation. Inserting this expression into (1) yields the desired form of the wave equation

$$
\frac{\partial^{2} \boldsymbol{E}(z)}{\partial z^{2}}+\left(k_{0}^{2} \varepsilon-\beta_{x}^{2}\right) \boldsymbol{E}(z)=0 .
$$

Naturally, a similar equation exists for the magnetic field $\mathbf{H}$.

We further assume that we are dealing with transverse magnetic (TM) modes, where only the field components $E_{x}, E_{z}$ and $H_{y}$ are nonzero. This choice is required to allow surface waves coupled to collective electron density oscillation produced by surface-free electrons, since this polarization imposes a discontinuity in the component of the electric field normal to the interface, leading to the abovementioned indispensable surface charge density. Thus, no surface modes exist for TE polarization. SPPs only exist for TM polarization. 


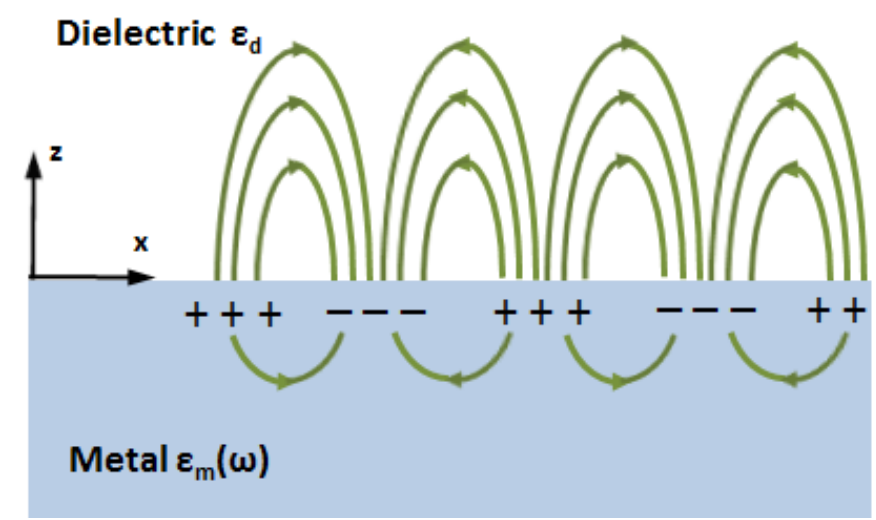

\section{Dielectric}

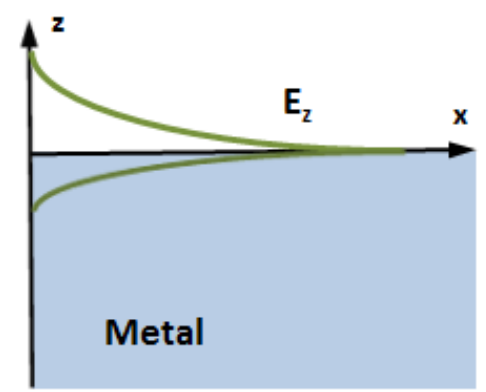

Figure 4. Geometry and electric field (left) and amplitude of $\mathrm{E}_{\mathrm{z}}$ (right) for SPPs propagation at a single insulator-metal interface. The field strength decreases exponentially with the distance from the surface. + and - represent the regions with lower and higher electron density, respectively.

In the case of a single flat interface between a non-absorbing dielectric medium and a conductor (Fig. 4), the system of governing equations for TM modes can be described by

$$
\begin{aligned}
& H_{y}(z)=A e^{i \beta_{x} x} e^{-k_{d} z} \\
& E_{x}(z)=\frac{i A k_{d}}{\omega \varepsilon_{d}} e^{i \beta_{x} x} e^{-k_{d} z} \\
& E_{z}(z)=-\frac{A \beta_{x}}{\omega \varepsilon_{d}} e^{i \beta_{x} x} e^{-k_{d} z}
\end{aligned}
$$

for $z>0$ and

$$
\begin{aligned}
& H_{y}(z)=A e^{i \beta_{x} x} e^{k_{m} z} \\
& E_{x}(z)=-\frac{i A k_{m}}{\omega \varepsilon_{m}} e^{i \beta_{x} x} e^{k_{m} z} \\
& E_{z}(z)=-\frac{A \beta_{x}}{\omega \varepsilon_{m}} e^{i \beta_{x} x} e^{k_{m} z}
\end{aligned}
$$

for $z<0$, since we want to look for solutions of propagating waves confined to the interface. Once we have the electromagnetic field components, applying continuity of tangential components at the interface $(z=0)$ and taking in mind that $H_{y}$ further has to fulfill the wave equation (2), the dispersion relation that governs an SPPs propagating at a single flat insulator-metal interface can be derived

$$
\beta_{x}=k_{0} \sqrt{\frac{\varepsilon_{d} \varepsilon_{m}}{\varepsilon_{d}+\varepsilon_{m}}} .
$$

The electromagnetic fields associated to the SPP supported in the considered geometry are shown in Fig. 4.

The SPPs' bound nature, which leads to an evanescent decay of the electromagnetic fields on both sides of the interface, can be inferred from eq. (5) since $\beta_{x}$ is larger than the dielectric's wave vector $k$ of the same energy and, 


\section{SURFACE PLASMONS}

therefore, the SPP dispersion relation lies to the right of the dielectric's light line (given by $\omega=c k$ ), ranging from zero (at $\beta_{x}=0$ ) towards the asymptotic value $\omega_{s p}=\omega / \sqrt{2}$ if a Drude model with negligible damping for the metal is considered, as depicted in Fig. 5. Therefore, the projection along the interface of the momentum $k_{x}=k \sin \varphi$ of photons impinging under an angle $\varphi$ to the surface normal is always smaller than the SPP propagation constant $\beta_{x}$, even at grazing incidence, prohibiting phase-matching between the incoming light and SPPs. Hence, SPPs in an ideal semi-infinite medium are nonradiative in nature, i.e. cannot decay by emitting a photon and, conversely, light incident on an ideal surface cannot excite SPPs. Thus, special phase-matching techniques to enhance the momentum of incident light such as grating or prism coupling are required for their excitation.

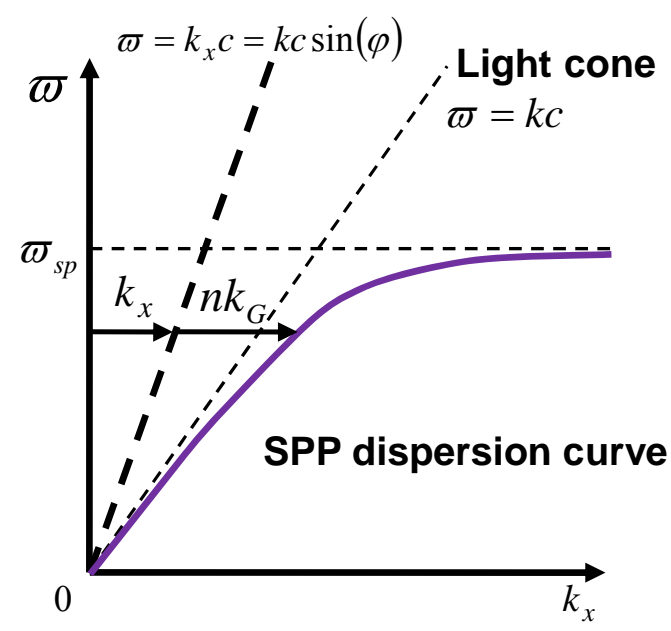

Figure 5. Dispersion relation of SPPs at a single metal-insulator interface.

In the prism scheme, phase-matching to SPPs can be achieved in a threelayer system consisting of a thin metal film sandwiched between two insulators of different dielectric constant, with the insulator of higher dielectric constant usually in the form of a prism. This coupling scheme, based on attenuated total internal reflection, involves tunneling of the fields to the interface between the metal and the lower-index dielectric where SPP excitation takes place. Two different geometries for prism coupling are possible, depicted in Fig. 6, (left) the Kretschmannn method, in which a thin metal film is evaporated on top of a prism [KRE-68], (middle) and the Otto configuration, in which the prism is separated from the metal by a thin gap [OTT-68]. 


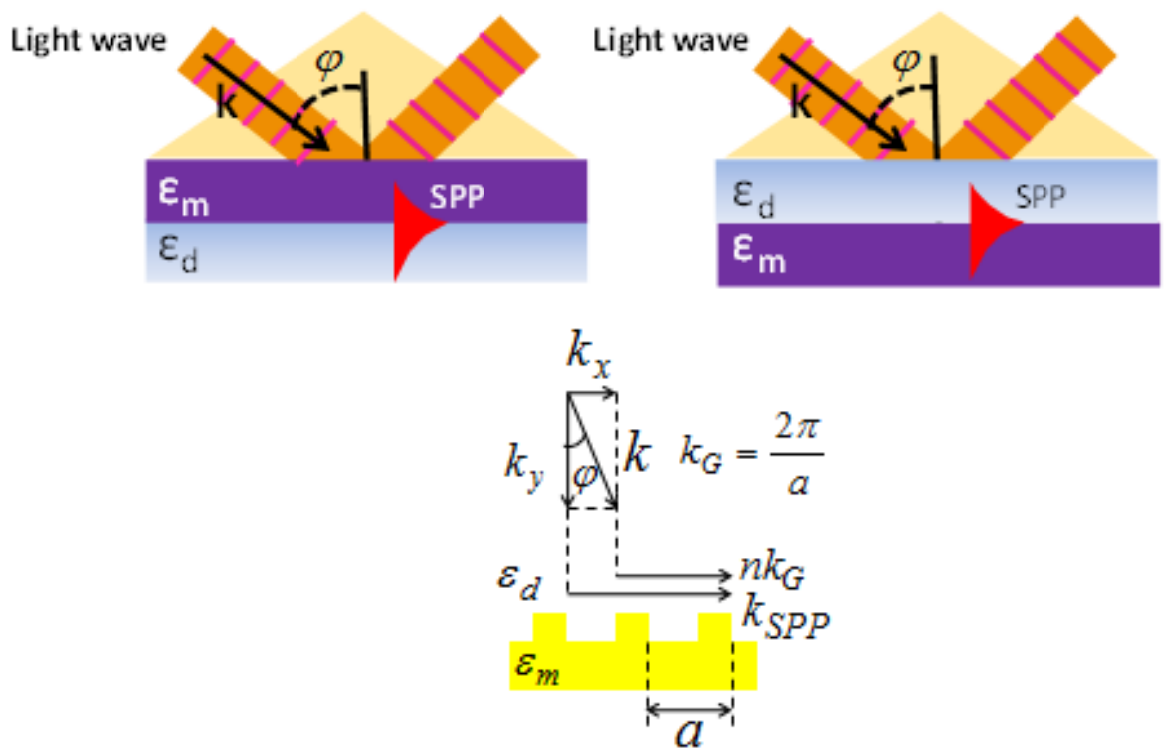

Figure 6. (Up): Prism coupling to SPPs in the Kretschmann (left) and Otto configuration (right). (Down) Phase-matching to SPP with a grating of lattice constant $a$.

The mismatch in wave vector between the in-plane momentum of the impinging light and SPP can also be overcome by periodically patterning the metal surface with grooves or holes with lattice constant $a$. For the simple case of one-dimensional grating depicted in Fig.6 (right), phase-matching takes place whenever the condition

$$
\beta_{x}=k \sin \varphi \pm n k_{G}
$$

is fulfilled, where $k_{G}=\frac{2 \pi}{a}$ is the reciprocal vector of the grating, and $n \in \mathbb{N}$.

The reverse process also takes place, and so SPPs propagating along a surface modulated with a grating can couple to light and thus radiate. These coupling and decoupling mechanisms play a key role in the onset of the extraordinary transmission effect [EBB-98].

\subsection{Metamaterials}

The index of refraction, defined as the square root of the product of relative electric permittivity $\varepsilon$ and magnetic permeability $\mu, n=\sqrt{\varepsilon \mu}$ is a fundamental parameter to describe the interaction of the electromagnetic radiation with matter. In the general case, this parameter is a complex number $n=n^{\prime}+i n^{\prime \prime}$ whose real part $n^{\prime}$ has been traditionally considered positive, although the 
condition $n^{\prime}<0$ does not violate any fundamental physical law. A negative refractive index is reasoned by a simultaneous negative permeability and negative permittivity. Furthermore, the media with a negative real index present unusual and astonishing electromagnetic properties. These properties were studied theoretically by Mandel'shtam [MAN-45] and Veselago [VES-68], albeit former works on negative phase velocity were carried out by Lamb [LAM-04], in the field of hydrodynamics, and Schuster [SCH-04] in optics. A historical review about these and other works can be found in [HOL-03].

As predicted by Veselago, in a negative index media backward wave propagation occurs and the phase velocity is anti-parallel to the group velocity. The phenomenon becomes visible in the dispersion diagram leading to a negative slope in the graph plotting the propagation constant versus frequency. Then, the electromagnetic field vector, the magnetic field vector and the wave vector form a left-handed $(\mathrm{LH})$ oriented system in contrast to the conventional right-handed $(\mathrm{RH})$ sense, hence the name left-handed media (LHM) to refer to this class of materials.

As a result, a reversal of Doppler and Cerenkov effects and Goos-Hänchen shift also occur in a LHM together with a frequency dispersion of the constitutive parameters and loss because of causality. However, the most interesting property, because of its important consequences, is the reversal of Snell's law. An electromagnetic wave impinging on a LHM from a conventional right-handed medium (RHM) will experience negative refraction causing the wave will not cross the normal, as represented in Fig. 7.

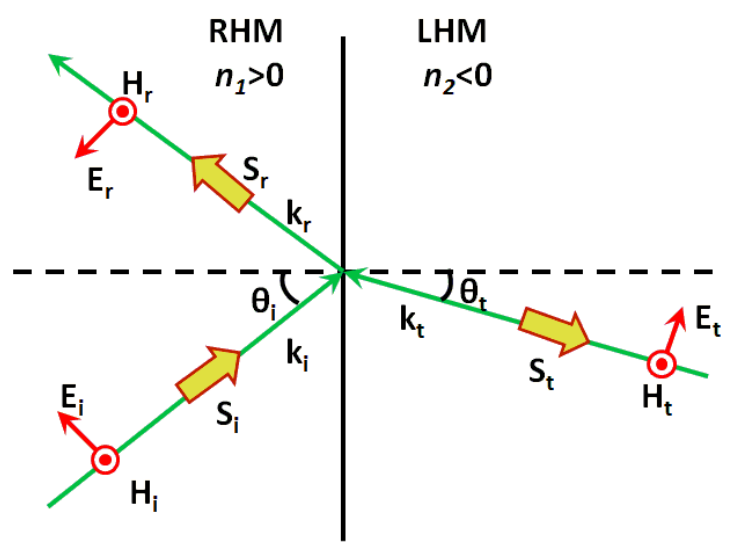

Figure 7. Negative refraction in a RHM - LHM interface. Because of the antiparallel direction between the phase and group velocities in a LHM, the impinging and transmitted waves remain in the same side of the normal as the tangential wave vector components have to be continuous along the interface. 
An important consequence of negative refraction is the possibility to obtain a perfect lens employing planar LHM slabs, see Fig. 8. Moreover, a significant aspect of this kind of lenses is their ability to obtain subwavelength focalization overcoming, therefore, the intrinsic diffraction limit of the conventional dielectric lenses. This extraordinary focusing effect, which allows theoretically a perfect imaging, relies in the amplification of the evanescent wave components through the excitation of SPPs [PEN-00]. However, the requirements for a perfect lens $\left(n^{\prime}<0, n^{\prime \prime}=0\right)$ are difficult to achieve. Whilst the former entails a dispersive refractive index $n(\omega)$ limiting the lens performance to a single frequency, the latter demands no absorption in the LHM slab. Actually, losses are always present in a LHM diminishing drastically the resolution [POD-05], [WEB-04], [SMI-03].
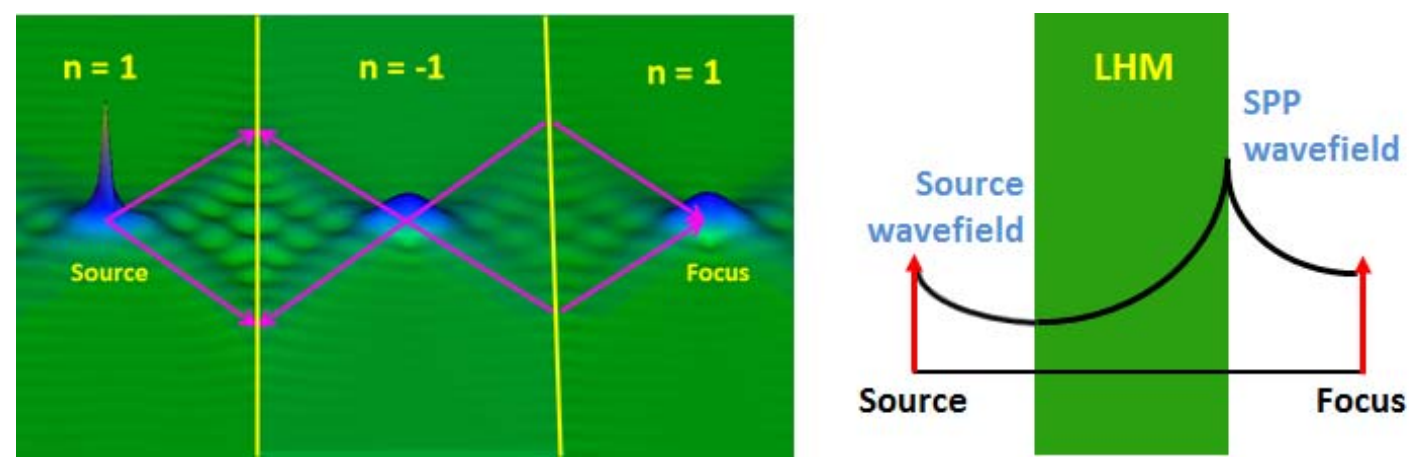

Figure 8. Focusing effect by means of a flat LHM lens. (left) A LHM bends light to a negative angle relative to the surface normal. Light formerly diverging from a point source in the object plane is set in reverse and converges back to a point. Released from the LHM the light reaches a focus for a second time in the image plane. The arrows indicate the wave propagation direction inside each medium. Simulation carried out by CST Microwave Studio. (right) The diagram shows an evanescent component of the source that is amplified by the slab, growing exponentially as a function of distance and then decaying exponentially until it reaches its original magnitude at the image.

A negative permittivity can be achieved with plasmas or metals, however the nonexistence in nature of magnetic monopoles prevent from obtaining negative permeability by a magnetic analogue of a plasma. Therefore, although the novel concepts resulting from research in LHMs generated considerable interest, the absence of any material realization led to their eventual neglect. It was not until some years ago when it was proposed that novel electromagnetic properties can be realized by structuring a material on a scale much less than the wavelength. By this manner, the first structure showing a negative magnetic permeability was designed [PEN-99b], which in combination with metallic wires 
with negative permittivity [PEN-96] allowed Smith's group to be the first who constructed and demonstrated an artificial material in which the sign of the permittivity and permeability were simultaneously negative [SMI-00], [SHE-01], verifying experimentally the negative refraction [SHE-01b].

These media were called thereafter metamaterials. And albeit, no definition exists that would be universally accepted, they can be defined as engineered composites that exhibit superior electromagnetic properties not found in nature and not observed in the constituent materials. These properties come as a result of the physical structure pattern rather than its chemical constitutive elements composition.

In contrast to photonic bandgap materials (also called photonic crystals) whose unit cell is comparable to the free-space wavelength and so are based on Bragg interaction, the ideal metamaterial contains many unit cells (or metaatoms) per free-space wavelength and therefore, in analogy to conventional materials where the permittivity and permeability derive from the response of constituent atoms to applied fields representing an average response of the system, a homogenization procedure can be applied to it. So on a length scale much greater than the separation between meta-atoms we may then ignore the details and pretend that there is no discrete structure (i.e. meta-atoms): the metamaterial is homogeneous and continuous and behaves as effective media defined by an effective electric permittivity and magnetic permeability parameters. In this way the properties of a complex structure can be summarized by $\varepsilon_{e f f}$ and $\mu_{e f f}$. The flexibility in design such meta-atoms enables metamaterials to have values for $\varepsilon_{e f f}, \mu_{\text {eff }}$ which are not encountered in nature and in the present context that will mean one or both of these parameters being negative. Furthermore metamaterials can provide magnetic activity at frequencies where previously materials have been thought of as magnetically inert. Depending on the effective parameters values, metamaterials can be sorted in single negative media (SNG), when only the effective permittivity or permeability is negative; or double negative media (DNM) (also termed as left-handed media (LHM), negative refractive index media (NRI), etc.), when both parameters are simultaneously negative. This classification is represented in the $\varepsilon-\mu$ diagram of Fig. 9. 


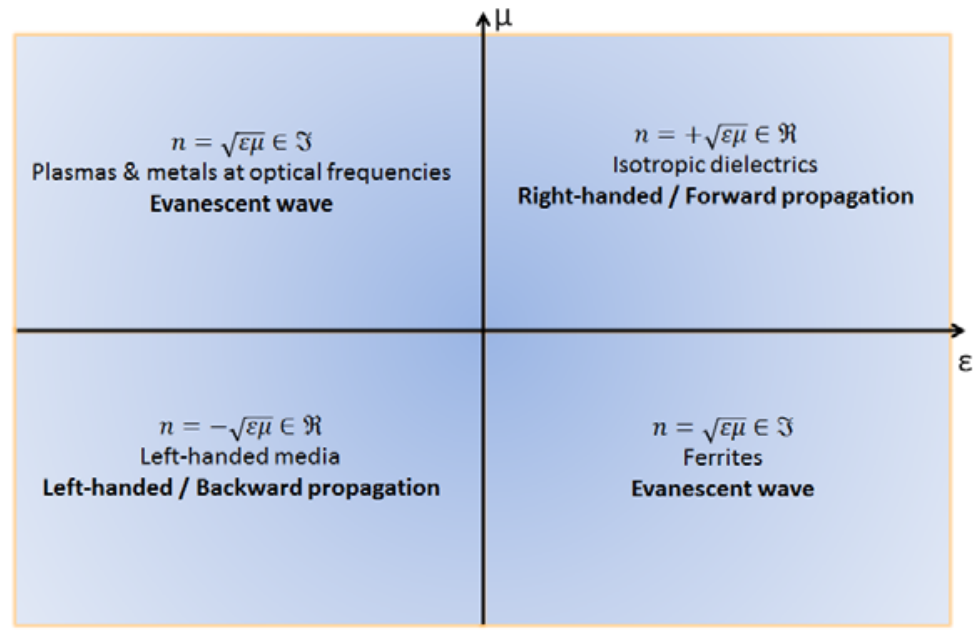

Figure 9. Permittivity-permeability diagram.

Since the first report on metamaterials, many studies were devoted to analyze the properties of this kind of media, as well as to implement them at higher frequencies scaling the structures down. In addition, in the optical range both the metal skin effect and the plasmonic resonances have to be taken into account. This is the reason why the design of new photonics metamaterials at optical frequencies is based on plasmonic effects. 



\section{Chapter 2}

\section{State of the art}

\subsection{A new phenomenon: Extraordinary transmission}

In 1998, Ebbesen and coworkers unveiled an astonishing experimental result related to anomalous light transmission through subwavelength aperture arrays drilled in optically thick metallic films [EBB-98]. They reported that in the cutoff region orders of magnitude more light than Bethe's prediction could be transmitted through the apertures. Specifically, under normal incidence, the transmission efficiency (normalized to the aperture area) of a single hole milled in an infinitely thin perfect conductor scales as $(r / \lambda)^{4}$, where $r$ is the hole radius and $\lambda$ the incoming light wavelength. Therefore, we would expect very low transmission efficiencies in subwavelength apertures due to the poor coupling of subwavelength holes to radiative electromagnetic modes [BET-44]. In addition, the transmission efficiency is further attenuated exponentially if the real depth of the hole is taken into account because of the evanescent decay of the electromagnetic fields inside the hole [ROB-87]. However, subwavelength aperture arrays exhibit transmission efficiencies (normalized to the total area of the holes) that exceed unity at wavelengths that the incoming radiation can only tunnel through the apertures in the transmission process. Representative pictures of these processes are depicted in Fig. 10. 

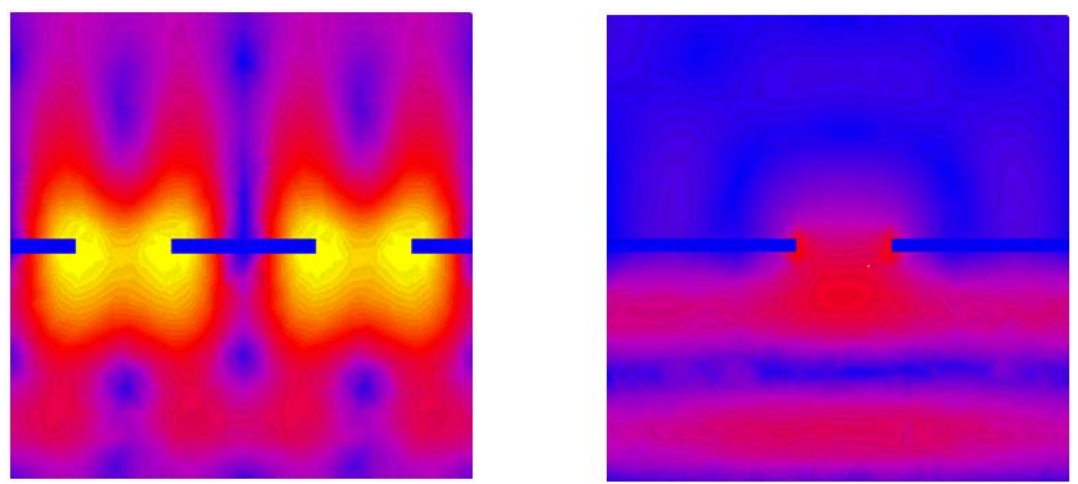

Figure 10. Simulation of transmission through a subwavelength hole array (left) and a single hole (right) in an optically thick metallic film. The color scale represents the electric field.

This phenomenon was named extraordinary optical transmission (EOT) since the discovery occurred at optical frequencies. Clearly, in light of these results, in the EOT the drilled metal film is not merely a screen that blocks the light, but rather an active participant in the transmission process. In other words, a collective response of the periodic array must occur in order to boost the transmission. Central to this interpretation is the role of surface waves such as SPPs. The combination of surface waves and subwavelength apertures is what distinguishes the EOT from the idealized Bethe treatment and gives rise to the enhancement. This has since sparked considerable interest in the scientific community and stimulated much fundamental research, due to not only their intriguing physical properties, in apparent contradiction with classical theories, but also to their potential applications from chemical sensors to atom optics and the design of very compact frequency selective surfaces (FSS), which has promoted subwavelength apertures as a core element of new optical devices.

It should be noted that thin metal films drilled with hole arrays were considered in the design of FSS long before the discovery of EOT [MUN-00]. However the principle of operation of those FSS called dichroic filters is inherently different to what occurs in the onset of EOT. In the dichroic filters the hole sizes are considerable large for those wavelengths at which there is transmission and the filtering properties arise by the low transmission for wavelengths longer than the cutoff wavelength $\lambda_{c}$ defined by the hole, whereas the lower transmitted wavelength is related with the redistribution of energy when a new diffraction order begins to propagate, thus, governed by the periodicity $d$. More precisely, dichroic filters act as band-pass filters for $d<\lambda<$ $\lambda_{c}$. However, the EOT presents two main differences. First, the metal films are optically thick, i.e., much larger than the skin depth of the metal. Second, the geometrical parameters defining the EOT structures are such that $\lambda_{c} \leq d<\lambda$, i.e., the holes are at cutoff when EOT occur. This is of the essence as, combined with the weak coupling of subwavelength holes to radiation modes, it is responsible for the appearance of narrow resonant peaks. 
Since the original experiments were performed in the optical regime, where SPP bounded to the metal surface can exist, the onset of EOT was attributed in a first moment to the excitation of SPP in the sculpted metallic film, which controls the coupling of the incident light and its scattering dynamics at the surface [EBB-98]. Many works were carried out to experimentally study the influence of Wood's anomaly, ascribing the minimum of transmission to the abovementioned anomaly due to diffraction effects [GHA-98], [KIM-99], the dielectric environment [KRI-01], the metal employed [GRU-00], the hole depth [DEG-02], and angle of incidence [BAR-04] so as to support their hypothesis based on SPP.

Also, interesting results were obtained with single apertures (holes or slits) surrounded by metallic grooves which harvest light efficiently and subsequently squeeze it through the apertures [THI-01], [GAR-03], [GAR-03b], [AKA-04], being remarkable the transmission enhancement achieved by an annular aperture in comparison with a circular aperture, due to the SPP coupling to the coaxial guided mode [CAG-05]. In the case that the metal presents a corrugation on the exit surface, the light pattern emerging from the aperture can be modified, leading to the beaming phenomenon, represented in Fig. 11 [LEZ-02], [MAR-03], [GAR-03b].

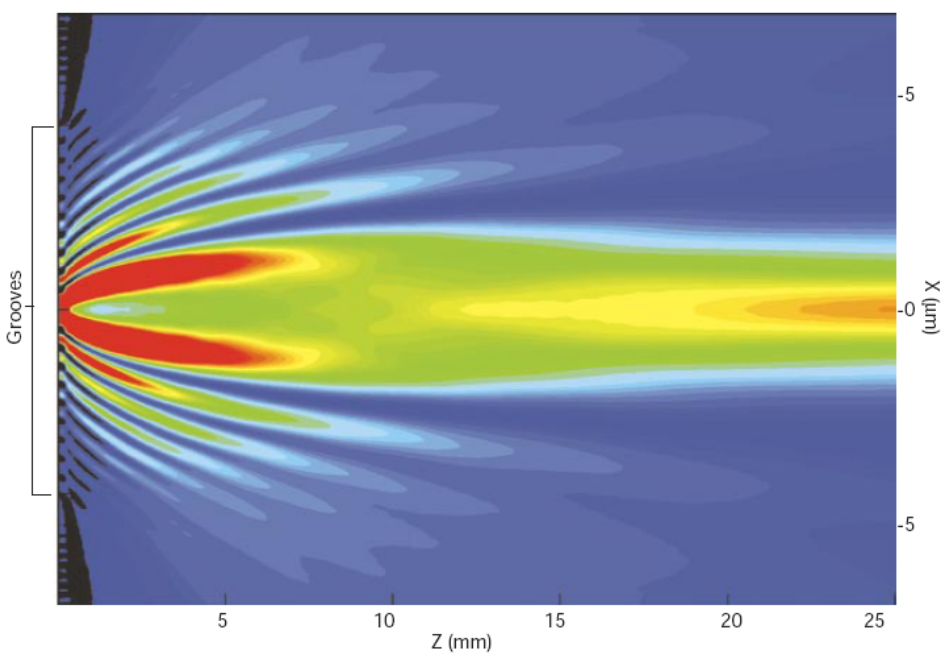

Figure 11. Calculated pattern of light emerging from a single slit flanked by a finite array of grooves. Image extracted from [BAR-03].

Many theoretical and experimental studies appeared based on different types of structures. Some of them questioned the SPP hypothesis or gave other perspectives to the underlying physics of the EOT. For instance, Cao et al. argued the negative role of SPP in the transmission since it is practically suppressed at those frequencies at which SPP excitation takes place [CAO-02]. It should be also mentioned the the dynamical light diffraction model proposed by Treacy [TRE-99], [TRE-02]. This model explains the EOT in terms of Bloch' waves arguing that the diffracted beams and SPP are part of the same multiple- 
scattering mechanism. These and other theoretical models [POR-99] were developed, because of its simplicity, from one-dimensional gratings formed by periodic array of slits. All of them showed the efficient light transmission of the slits due to its non-cutoff regime, condition inherently different to hole apertures, so the electromagnetic behavior of both structures is essentially different and the SPP theory of slit arrays may not be useful for explaining EOT on hole arrays. Subsequently, some studies were devoted to the study of the underlying physics of hole arrays. In this sense, Lezec et al. developed a model to explain the EOT in terms of the superposition of the diffracted evanescent waves generated by the subwavelength apertures at the metallic surface and their interference with the incoming electromagnetic field [LEZ-04]. An alternative method to study light transmission through hole arrays in perfect conductor metal sheets was relating it by Babinet's principle to the reflection on planar metallic disks arrays, which are solved from the multipolar polarizability of single disks [GAR-05]. Other studies stressed the role of multiple-scattering, emphasizing their work on Wood's anomalies [SAR-03] and Brewster-Zenneck modes [SAR-05], or focused on leaky waves [LOM-04], [LOM-05]. The important result is that it explains the EOT in any frequency range remarking the relevance of the periodicity and the surface waves whatever they are SPP or leaky waves.

Following with the theoretical models that are able to reproduce the EOT phenomenon relying on SPP, it should be mentioned the model developed by Avrutsky [AVR-00]. In this model, EOT is interpreted by a mechanism of - in his own words - plasmon-assisted light tunneling. The notable result is that it predicts EOT even in the absence of apertures, just being necessary a periodically corrugated metal. The first three-dimensional theoretical models about hole arrays appeared with the contributions of Popov et al. [POP-00] and MartínMoreno et al. [MAR-01]. However, they reached different conclusions. While [POP-00] ascribes the onset of EOT to a propagating mode appearing in the hole array as long as a realistic model of the permittivity is considered, in [MAR-01] the EOT is due to a tunneling of the SPPs excited in each dielectric-metal interfaces through an evanescent mode of the hole. The remarkable consequence of this model is that EOT can occur at any frequency range, even at those where SPP make no sense because of the metal behaves as a perfect conductor.

Finally, both theories were joined together with the theoretical framework of spoof surface plasmons [PEN-04]. Pendry et al. showed that surfaces waves in a perfect conductor sheet can mimic a SPP. However, the outstanding conclusion is that the effective permittivity of the subwavelength structures resembles a Drude model, whose plasma frequency can be tailored by the geometry of the apertures, allowing the inducement of SPP-like surface waves in any region of the spectrum where the metal sheet approximates to a perfect conductor. This theory was 
verified experimentally in the microwave range, showing the existence of a surface wave that emulates the SPP field pattern on a metal surface at optical frequencies [HIB-05].

Thus, despite their differences, all the theories incorporate implicitly common elements that lead to achieve essentially similar predictions. Consequently, there is a general agreement in which the coupling of the incoming radiation to a surface wave by means of the periodicity is essential for transmission enhancement via evanescent tunneling. In the optical range the surface waves take the form of SPP, whereas in the microwave or terahertz regimes surface waves result from the interaction with the apertures, even when the metal behaves as a perfect conductor.

On the other hand, theoretical [GAR-05b], [CHA-05], [POP-05], [WEB-06], [GAR-02] as well as experimental [DEG-04], [YIN-04] studies noted that the transmission of light through single apertures presents resonant peaks that can also overcome Bethe's prediction. The appearance of such resonances can be understood as the excitation of SPP at the edges of the hole, a type known as localized SPP. Such behavior is reminiscent of elongated metal particles, the colours of which are also determined by localized SPP. In addition, the fact that the shape of individual holes in arrays of subwavelength holes has a dramatic effect on the optical transmission through the films proves the interaction between SPP at the surface of the metal, on the one hand, and localized resonances inside the holes, on the other hand. As the localized modes effectively act as waveguides, this interaction increases the coupling efficiency between SPP from both sides of the film, resulting is a higher transmission. However, this is such an intricate interaction that makes the contributions of surface plasmons and localized modes to the EOT phenomenon cannot be trivially separated in two distinct contributions [MOL-05], [GAR-06]. Whereas the localized SPP are defined by the lateral dimensions of the aperture, theoretical studies showed that in addition to such SPP modes other resonant modes defined along the depth of the hole [GAR-05b], [GAR-06b] might also be present and contribute to the onset of EOT.

Due to the role of the periodicity in the onset of EOT, it seems evident the critical factor that the physical size of the array may have in the electromagnetic response of the structures. The influence of the inherent finite size of the arrays became obvious when numerical results of infinite arrays are compared with experimental values of real structures. These intrinsic finite structure effects were studied theoretically in [BRA-04]. The main consequence in the transmission properties of finite arrays is a lowering of the spectral resolution. As a result the finest spectral details appearing in the infinite array will smooth in a real 
configuration and the transmission level will fall drastically. As well as, Wood's anomaly, which in infinite arrays appears as an abrupt transmission drop, will become soften in finite arrays. Corroborative experimental results show how the transmission raises at the frequency of resonance when the number of holes drilled in the array increases, reaching a transmission maximum when the array consist of 31x31 holes [BER-04], [BER-05], [BER-05b]. Similar results were obtained in configurations of slits and grooves [GAR-03]. Moreover, it was noted the strongly anisotropic and extremely sensitive to the angle of incidence of the impinging light behavior of the spatial distribution of light as it emerges from the arrays [BRA-06].

Finally, it is worth mentioning that even though structural periodicity is perceived to be crucial in forming the transmission resonances, EOT phenomenon was also theoretically [BRA-07] and experimentally [PRZ-06], [MAT-07] demonstrated in non-periodical Penrose structures, see Fig. 12. Specifically, EOT will take place at those frequencies which wavevectors match the corresponding discrete Fourier transform vectors. These results expand potential design parameters for subwavelength structures that are aperiodic but contain discrete Fourier transform vectors.
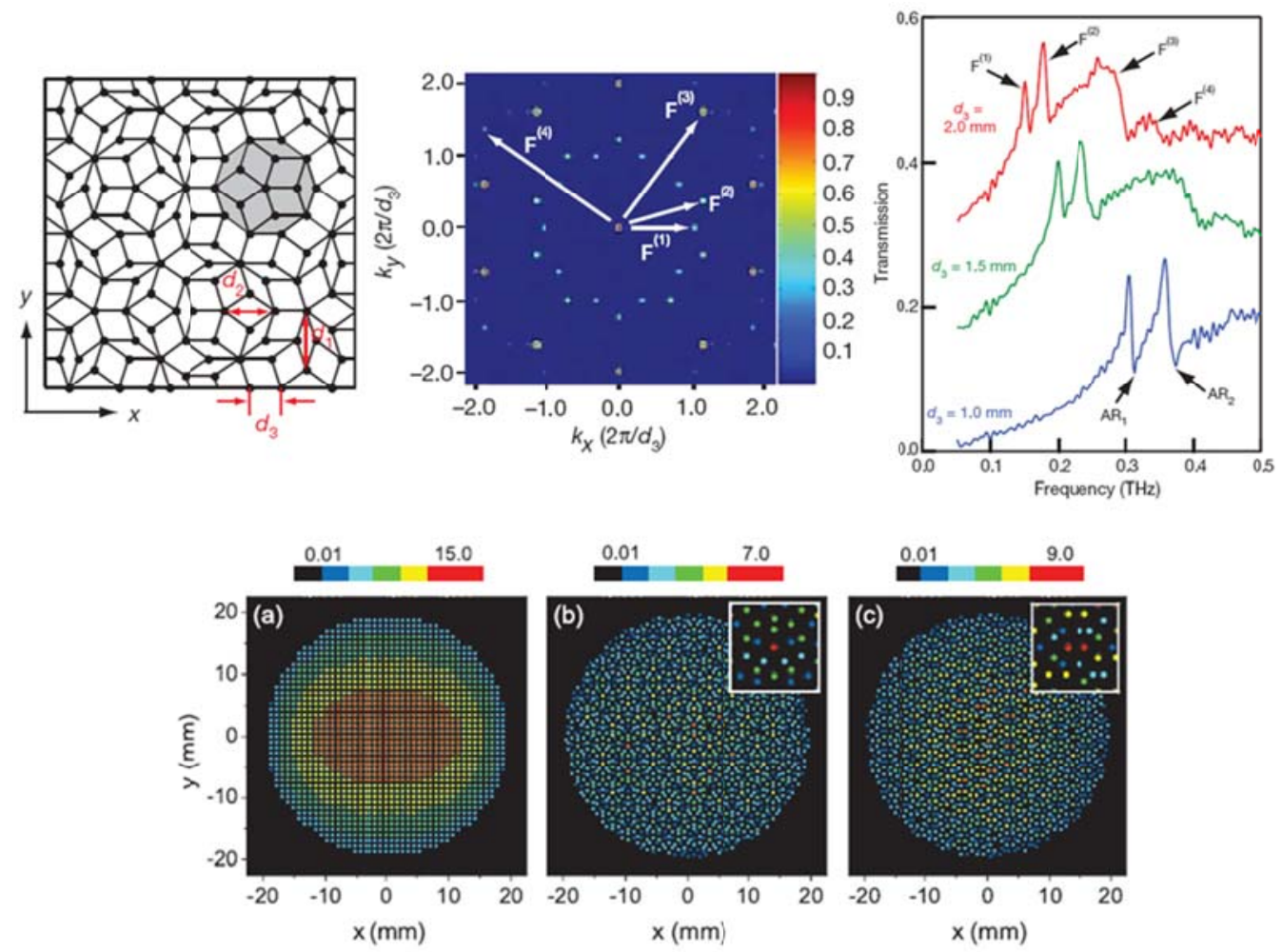

Figure 12. (Up): Penrose structure with five-fold rotational symmetry (shady region) with apertures at the vertexes. (left), its reciprocal Fourier space with the corresponding discrete vectors $\mathrm{F}^{(\mathrm{i})}$ (middle) and the transmission spectrum with their resonances $\mathrm{F}^{(\mathrm{i})}$ and antiresonances $\mathrm{AR}_{\mathrm{i}}$. Images extracted from [MAT-07]. (Down): Transmission pattern through an array with square periodicity (a) and Penrose structure for two different frequencies (b)-(c). Image extracted from [BRA-07]. 
Despite the theoretical and experimental works carried out by Economou [ECO-69] and Kovacs [KOV-77] respectively, the study of EOT has been traditionally focused on single metallic films structures. However, it was not until recently when much more attention has been paid to multilayered metal structures. In doing so, EOT has been shown through a couple of unpatterned metal sheets [WAN-03] or drilled with isotropic [MIY-05], [YE-05] or anisotropic hole arrays, as well as in a bilayer structure drilled with slits [HIB-04], [CHA-06]. Finally, many works have also been focused on hole arrays milled in stacks of multiple metallic films [TAN-07], [BER-06], [BER-07], [NAV-08], [MAR-08]. In such configurations the excitation of SPP among the metallic films contributes to the onset of the EOT [MAR-08]. One interesting characteristic of the stacked arrays is that they may present EOT together with a negative index of refraction [BER-06], [BER-07], [NAV-08], [MAR-08], [GAR-09], [GAR-09b], [GAR-11] connecting the world of plasmonics with that of metamaterials. In fact, the existing relationship between the excitation of SPP and the achievement of a negative refractive index in metallic stacked hole arrays allows us to model, in a very suitable way, the behavior of metamaterials based on the fishnet structure [ZHA-05], [ZHA-05b], [DOL-06], [DOL-06b], [Li-06], [LI-07], [LI-07b]

\subsection{Negative refractive metamaterials}

The history of effective metamaterials dates back to beginning of artificial dielectric and magnetic media. Although the former were known as far back as mid $20^{\text {th }}$ century, artificial magnetic media did not boost their interest until the end of the $20^{\text {th }}$ century, with the finding of the magnetic response provided by Split-Ring Resonators (SRRs) [PEN-99b] (see Fig. 13). This crucial breakthrough paved the way to achieve tailored electric and magnetic properties by means of engineered subwavelength structures, which when gathered together, could lead to a medium with simultaneous negative values of the effective electric permittivity and magnetic permeability. It was the birth of negative refractive metamaterials.

The first designs of negative index metamaterials were based on the combination of SRRs with arrays of parallel wires [SMI-00], [SHE-01], depicted on Fig. 13. The subwavelength SRRs particle gives rise to a resonant effective permeability following a Lorentz response due to the induced local magnetic moment which counteracts with the incoming magnetic field [PEN-99b]. On the other hand, the parallel wires array is responsible for the Drude response of the effective permittivity [PEN-96], [PEN-98]. Consequently, the novel man-made composite, i.e. metamaterial, resulting from the combination of both 
subwavelength structures showed a negative refractive index as verified experimentally [SHE-01b].
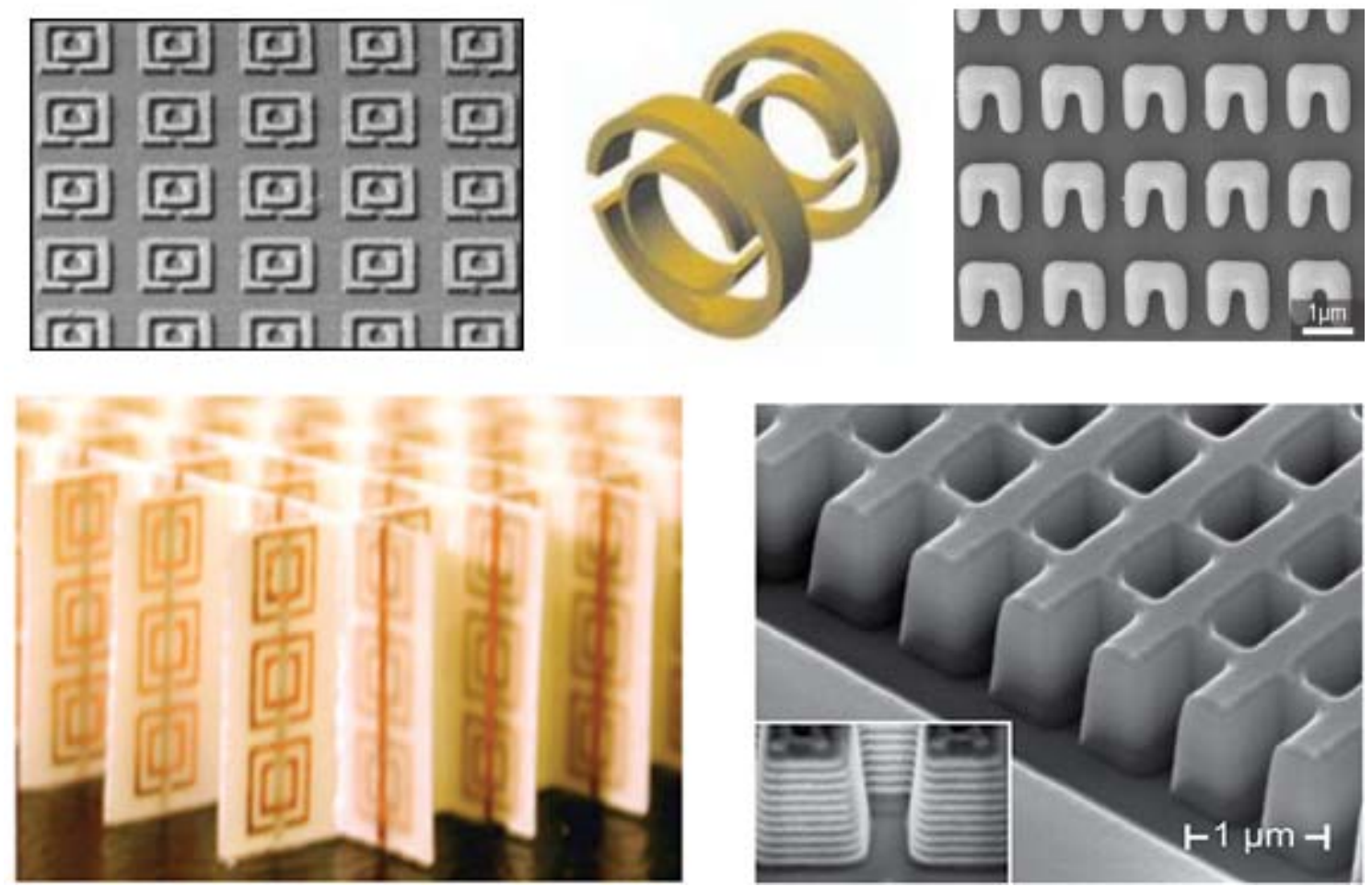

Figure 13. (Up): Sketch of a SRR particle (middle) alongside SEM images of samples fabricated. (Down) First implementation of a negative index metamaterial constructed with SRR and metal wires -image extracted from [SHE-01b] (left) and a stacked fishnet metamaterial (right) extracted from [VAL-08]-.

However, the inherently high losses and narrow band response -due to, principally, the resonant behavior of the SRRs- prevent such metamaterials from their implementation at high frequency regimes, particularly at optical frequencies $(>>100 \mathrm{THz})$ where metal losses become significant. Hence, many alternative routes were followed to circumvent these issues and take advantage of the potential applications of LHM in the optical regime. Apart from the inverse transmission line formalism [CAL-05], [ELE-05], which needs to use lumped elements --that, once again, hinder their implementation at optical regimes- and from more suitable structures to optical frequencies but based on the classical double-resonance scheme [SHA-05] which increases losses, the first attempt to elude high losses at optical frequencies relied on the fabrication of a pair of subwavelength hole arrays milled in metallic plates [ZHA-05], [ZHA-05b], [DOL06], [DOL-06b], (see Fig. 13).

This perforated metal-dielectric-metal sandwich structure, also known as fishnet, has been used by several groups [ZHA-05], [BER-06], [DOL-06], [DOL06b], [DOL-07], [CHE-07], [VAL-08], [GAR-09b], [XIA-09], [GAR-11] from microwave to optical frequencies. It has been demonstrated that it can exhibit an 
effective negative index of refraction for light propagating normal to the perforated layers. The next step is to stack several functional layers of the fishnet structure to achieve "bulk" properties. Bulk metamaterial properties are reached if the retrieved effective parameters [SMI-02], [KRI-10], [KU-09] converge with an increasing number of layers. Obviously, the minimum number of layers required for the metamaterial to qualify as bulk depends on both the structure under investigation and the coupling between adjacent layers, and hence on the interlayer spacing, i.e. the unit cell size along the propagation direction. One simple approach to prove the experimental evidence of the optical metamaterial properties is by comparison of the measured transmission and reflection coefficients with numerical results, which are then used to retrieve the effective parameters provided that the agreement between the experiment and theory is excellent. By this manner, several groups have provided not only reliable evidence of the uniaxial negative index of the fishnet metamaterial [GAR-11], but also the negative permeability of the SRRs at different frequencies [KAT-05], [MIY-10], [LIU-08]. Other methods to prove experimentally the negative index of the fishnet is demonstrating the negative refraction in a wedge prism [VAL-08], [SHE-01b] or directly measuring the negative phase velocity of light in an interferometric configuration [DOL-06b], [DOL-07].

However, a major drawback appears when moving towards the bulk case as the metamaterial losses become an increasingly important issue that can render the metamaterial essentially opaque and hence useless. The metamaterial's loss can be quantified by means of the dimensionless figure of merit (FOM), related to the refractive index by $F O M=n^{\prime} / n^{\prime \prime}$. The best experiments of passive fishnet metamaterials have given a FOM=3 [GAR-11], [DOL-07b]. Such large losses come as a result that the negative index region is close to the resonance of the fishnet unit cell, where $n^{\prime \prime}$ is large. Although future improvements on FOM values can be achieved with optimized designs, the desired increased of several orders of magnitude seems to be out of reach, especially at optical wavelengths. Obviously, losses can be compensated by introducing active materials into the metamaterial structure. Although this approach will employ large gain coefficients, which are difficult to obtain in practice [SOU-10], it seems not impossible to achieve [XIA10]. Alternatively, instead of metal-based metamaterials that leads to inherently large losses, purely dielectric off-resonant constitutive materials can be employed to circumvent losses. Based on Mie resonances, dielectric particles can show both negative magnetic permeabilities and electric permittivities, which enables several alternative techniques for realizing low-loss isotropic negative index metamaterials in the optical regime [SOU-11].

Another challenge in the metamaterials field to achieve operation at very short wavelengths is the fabrication of very fine nanostructures such that their 
period is still well below the optical wavelength (within the medium, not just in vacuum). Some lithographic techniques, such as standard electron-beam lithography, focused-ion-beam lithography, interference lithography and nanoimprint lithography, are just sufficient to fulfill that condition, partly by using shorter wavelengths for the fabrication and partly by achieving a subwavelength resolution. Other techniques such as stacked electron-beam lithography, direct laser writing, electroplating or membrane projection lithography can also be employed, especially when functionalities other than negative refractive index are desired and flexibility in tailoring a metamaterial's unit cell interior is a crucial factor, like for instance in chiral media or transformation optics which requires generally tailored anisotropic magnetodielectric metamaterials. The speed of such fabrication methods may limit the size of the parts made within a reasonable time. 


\section{Chapter 3}

\section{EOT and negative index in fishnet}

\section{metamaterials}

As mentioned previously, while most of the demonstrated NIMs working in the microwave regime are systems based on split-ring resonators in combination with a metallic wire medium, the fishnet metamaterial is the most common NIM design employed at optical frequencies due to its relative low losses and easily fabrication compared to other metamaterials designs. This is the reason why this chapter is devoted to studying analytically and numerically the far-field EOT through double-layer metallic grating structures patterned with subwavelength hole arrays. In addition to EOT phenomena due to the well-known SPPs on the outer surfaces such as those on a single layer hole array, further EOT peaks are observed coming from the excitation of SPP in the inner interfaces. A relatively simple model to predict the frequencies of those EOT peaks is proposed. Moreover, internal SPPs show certain unique properties different from the external SPPs: they can give rise to a magnetic response and a negative effective permeability. All these findings may be utilized in wavelength tuning of extraordinary optical transmission in subwavelength optics.

\subsection{Introduction}

Since 1998, when Ebbesen [EBB-98] published their pioneering work about EOT through hole arrays in metallic films, many studies have proved the existence of EOT. Most studies have focused on single-layer metal films drilled with periodic hole arrays, being well accepted that the EOT through single-layer hole arrays originates from the grating coupling between the dispersion relation of the SPPs that propagate along a smooth infinite metal-dielectric interface and the 
momentum of the incident light taking into account the lattice structure provided by the holes. However, this procedure has to be considered as an approximation due to the fact that it models the momentum of the SPPs on a hole array using the expression of SPPs on a smooth metal-dielectric interface, thus ignoring the effect which the holes may have on the SPP propagation. Nevertheless, the predictions of this model are usually very accurate [MAR-01], [GEN-07], [GRU00], [THI-99], [DEG-02], [GHA-98], [RAE-86].

Though multilayers structures were previously studied theoretically [ECO69] and experimentally [KOV-77], EOT studies have been focused traditionally on single-layer structures. It is until quite recently when studies of multilayer hole arrays have gained more attention. In this way, transmission through double-layer smooth surfaces [WAN-03] or drilled with slits [HIB-04], [CHA-06] or hole arrays with isotropic [MIY-05], [YE-05] as well as anisotropic [LI-08] shapes have been studied. Multilayer structures have also been studied [TAN-07] showing, in addition, a left-handed behavior when stacking subwavelength hole array plates [BER-06], [BER-07], [NAV-08], [MAR-08], [GAR-09], [GAR-09b], [GAR-11].

In this chapter, the fishnet structure is studied both analytically and numerically. As a result, a simple approximate model, in analogy with the model for single-layer hole arrays, is developed. This analytical model allows for predicting an approximate frequency for all the EOT peaks coming from the excitation of SPPs on the fishnet structure. Additionally, it deserved to be mentioned that a great relationship between the world of plasmonics with that of metamaterials exists as SPPs play a fundamental role in achieving a negative effective permeability on double layer hole arrays, as the associated electromagnetic field forms a virtual current loop between the two metallic layers. In fact, the existing relationship between the excitation of SPP and the achievement of a negative refractive index in metallic stacked hole arrays allows us to model, in a very suitable way, the behavior of metamaterials based on the fishnet structure [ZHA-05], [ZHA-05b], [DOL-06], [DOL-06b], [LI-06], [LI-07], [LI07b].

\subsection{Numerical results}

First of all, the SPPs dispersion relation propagating on the multilayer geometry without holes depicted in Fig. 1 is calculated for the general case in which all the layers have an arbitrary electric permittivity. To this end, only TM modes are considered as it is widely known that SPPs does not exist for TE polarization [MAI-07]. In the assumed one-dimensional problem, the electromagnetic field in each medium can be expressed according to Eqs. (1)-(8), where coupled propagating wave solutions with an evanescent decay in the direction normal to 
the interfaces are considered. Then, the implicit dispersion relation is obtained by solving the linear equation system enforcing the continuity of the tangential EM components at every interface separating two media.

The SPPs dispersion relation given by Eq. 9 and plotted in Fig. 2 accounts for two types of SPP modes decoupled from each other, namely, the internal SPP and the external SPP which propagate along the internal and the external metaldielectric interfaces, respectively[ECO-69].

Although Eq. 9 only describes the fundamentals of SPPs in a smooth double-layer metallic structure, it can be considered, in analogy to what is done for single-layer structures, that the SPP dispersion relation on a double-layer hole array will be similar to that obtained in the smooth structure. This procedure allows us to predict analytically all the EOT peaks coming from the excitation of both internal- and external-SPP modes at those wavelengths at which they couple to the incoming light via grating coupling, given by Eq. 10. Indeed, this model permits a complete identification, classification, and enumeration of such peaks.

To check the validity of the model, numerical calculations are performed to investigate the transmission through a double-metallic layer, which unit cell is depicted in Fig. 3. The normalized transmission spectrum is compared to the dispersion relation given by Eq. 9 in Fig. 4 for different dielectric permittivities $\varepsilon=1$ and $\varepsilon=4$. The predicted EOT peak frequencies calculated by equating the matching condition given by Eq. 10 with the full dispersion relation of Eq. 9 (solid lines) are shown for different values of $(i, j)$ of both internal and external SPPs. The predicted frequencies are seen to approximately match the simulated spectra EOT peaks (dashed lines). Agreement between simulation and our model is not perfect, in the same way as in a single-layer hole array, because the dispersion relation of the SPPs used as matching condition does not take into account the presence of the holes, which cause scattering losses and a resonance shift. As a consequence, the predicted resonant frequencies are slightly larger than those obtained in simulations [GEN-07]. On the other hand, the transmission minima (dotted lines) are the result of Wood's anomaly [WOO-02], [WOO-35] and their positions are in a good agreement with the minima positions of the simulated spectra.

Now, if one takes a look to the electromagnetic profiles of each resonance, it can be seen how for the external SPPs resonances, the electromagnetic fields are located on the outer metal-air interfaces, being partially concentrated at the edge of the holes (see Fig.5), whereas for the internal SPPs the electromagnetic fields are clearly concentrated on the internal dielectric layer as shown in Fig.6. Thus, these field patterns fully match all the electromagnetic field features expected from the theoretical model. 
Considering the subwavelength thickness of the structure, the effective constituent optical parameters are retrieved from the transmitted and reflected spectra [SMI-05] shown in Fig. 7. The retrieved $\varepsilon_{e f f}$ is negative below the first external-SPP EOT peak, whilst_ $\mu_{\text {eff }}$ becomes negative at the internal-SPP resonances. This magnetic negative response comes as a result of the antiparallel currents, excited at opposite internal metallic interfaces, which along with the displacement currents give rise to a virtual current loop on a perpendicular plane to the incoming magnetic field [HUA-06]. Due to their analogy to a LC circuit, the internal-SPP resonances have been sometimes called LC resonances [ZHA-05] -in this way, an expression linking the resonant frequencies of the internal SPP with that of the VCL equivalent circuit can be derived (see Eq. 14)- coming from a magnetic plasmon polariton (MPP) but without further indication of its real physical origin [LI-07], [LI-07b], [LI-06]. However, the presented model gives a complete interpretation of the magnetic resonance ascribed to internal SPP excitations, even for higher order internal-SPPs modes usually termed high order MPPs, [LI-07], [LI-07b] which can be clearly identified and enumerated with the proposed model. Indeed, the presented relationship between the internal-SPP EOT peaks and its resonant negative magnetic response in double-layer hole array is, in fact, a very appropriate way of modeling the well-known fishnet metamaterial.

Therefore, if the internal-SPP resonances occur below the external-SPP resonance, negative $\varepsilon_{e f f}$ and $\mu_{e f f}$ coincide in frequency and a negative effective index $n_{e f f}$ is retrieved, thus, achieving an EOT with a negative effective index nature [GAR-09].

Finally, the dependence of the spectral response on the dielectric parameters (thickness and permittivity) and on a lateral displacement of the metallic layers are also studied. The results (see Figs. 8-10) show that the external SPP resonant frequency displacement is highly determined by the structure design and its related electromagnetic field distribution and can be taken into account with a suitable equivalent permittivity which depends on the dielectric parameters. On the other hand, the transmission remains remarkably large at resonance, even though in the case of no direct line of sight through the structure due to the matching of the Poynting vector between the layers [MIY05].

\subsection{Conclusions}

The dispersion relation of SPPs in a double-layer metallic structure is obtained analytically and proved by numerical analysis, showing a good agreement with theory, that light coupling to the external and internal SPPs originates EOT. In 
addition, at the internal-SPP resonant frequencies a negative effective permeability is achieved as a VCL is formed between the metallic layers which can be used to design negative-index metamaterials.

Moreover, the influence of both the sandwiched dielectric layer parameters and the alignment between holes in the transmission resonances is presented. Showing a remarkably large transmission even in the case of no direct line of sight. These effects can be used to tune the resonant frequencies for both the internal and external SPPs.

The model presented here surely facilitates the interpretation and design of double-layer structures, such as the fishnet metamaterial. 


\title{
Role of surface plasmon polaritons on optical transmission through double layer metallic hole arrays
}

\author{
R. Ortuño, * C. García-Meca, F. J. Rodríguez-Fortuño, J. Martí, and Alejandro Martínez \\ Valencia Nanophotonics Technology Center, Universidad Politécnica de Valencia, Campus del Camino de Vera, 46022 Valencia, Spain
}

(Received 28 August 2008; revised manuscript received 14 November 2008; published 12 February 2009)

\begin{abstract}
We study analytically and numerically the far-field extraordinary optical transmission (EOT) through double-layer metallic grating structures patterned with subwavelength hole arrays. In addition to EOT phenomena due to the well-known surface plasmon polaritons (SPPs) on the outer surfaces such as those on a single layer hole array, further EOT peaks are observed. The separation between the metallic layers is small enough to allow SPPs propagating through the inner interfaces to couple and form an internal SPP, with a different dispersion relation from the outer one and so giving rise to EOT peaks at different frequencies. We propose a relatively simple model to predict the frequencies of those EOT peaks. Internal SPPs show certain unique properties different from the external SPPs: they can give rise to a magnetic response and a negative effective permeability, and the transmission of their EOT peaks increases when no direct line of sight is allowed through the structure. All these findings may be utilized in wavelength tuning of extraordinary optical transmission in subwavelength optics.
\end{abstract}

DOI: 10.1103/PhysRevB.79.075425

PACS number(s): 73.20.Mf, 71.36.+c, 78.66.Bz, 42.79.Dj

\section{INTRODUCTION}

A surface plasmon polariton (SPP) is an electromagnetic surface wave traveling along the interface separating a dielectric and a metal. It is produced by the interaction of collective oscillations of free electrons in the metal surface with the electromagnetic wave impinging on the metal. The first theoretical description of surface plasmons dates back to 1957 when Ritchie ${ }^{1}$ predicted the existence of surface plasmons when he discussed the plasma losses in thin films. However, it was in 1998, when Ebbesen et al. ${ }^{2}$ published their pioneering work about extraordinary optical transmission (EOT) through hole arrays in metallic films, when SPPs attracted the attention of fundamental research giving rise to the field of plasmonics. Since then, other studies have proved the existence of EOT, mostly on single-layer metal films with periodic hole arrays, being well accepted that the EOT through single-layer hole arrays originates from the grating coupling between the dispersion relation of the SPPs that propagate along a smooth infinite metal-dielectric interface and the momentum of the incident light taking into account the lattice structure provided by the holes. However, this procedure has to be considered as an approximation due to the fact that it models the momentum of the SPPs on a hole array using the expression of SPPs on a smooth metaldielectric interface, thus ignoring the effect which the holes may have on the SPP propagation. Nevertheless the predictions of this model are usually very accurate..$^{3-6}$

Though theoretical modeling of multilayers was studied by Economou ${ }^{7}$ and following experiments by Kovacs, ${ }^{8}$ EOT studies have been focused traditionally on single-layer structures. It is until quite recently when studies of multilayer hole arrays have gained more attention. In this way, transmission through double-layer smooth surfaces ${ }^{9}$ or drilled with hole arrays with isotropic ${ }^{10,11}$ as well as anisotropic ${ }^{12}$ shapes have been studied. Also, the electromagnetic responses of double-layer metal structures perforated with an array of slits ${ }^{13,14}$ and near-field optical images of double- layer nanoparticles ${ }^{15}$ have been explored. Multilayer structures have also been studied ${ }^{16}$ showing, in addition, a lefthanded behavior when stacking subwavelength hole array plates. $^{17}$

It is known that when two metal layers are placed closer than the SPP attenuation length, the SPPs that propagate along each of the two inner interfaces couple to each other, thus creating a mode called internal SPPs whose dispersion relation differs from the usual aforementioned dispersion relation of the SPPs on a single interface. Thus, EOT peaks are observed at frequencies different from those expected using the model of SPPs on a single metal-dielectric interface.

In this work, we study both analytically and numerically a structure composed of a dielectric layer sandwiched between two metal layers nanostructured with subwavelength hole arrays. Comparing our work with previous results, we emphasize that we have developed a simple approximate model, in analogy with the model for single-layer hole arrays, to predict analytically an approximate frequency for all the EOT peaks coming from the excitation of SPPs on a double-layer hole array structure. To this end, we consider, in analogy with the model for single-layer hole arrays, the dispersion relation of the SPPs propagating on a multilayer structure without holes and equate it with the momentum of the incident light taking into account the periodicity of the structure provided by the holes.

In the simulations we vary various parameters such as the dielectric thickness and its permittivity to study its influence in the transmission spectra. A good agreement between the wavelengths at which EOT occur in simulations and those expected from the calculated SPP dispersion relation via grating coupling is found. From the dispersion relation of the SPPs propagating on a multilayer structure without holes, it can be seen that two types of resonances, internal and external SPPs, are responsible for the EOT. The internal SPP resonates in the inner surfaces of the metal layers, whereas the external SPP resonates in the outer surfaces of the metal layers. 
From a different perspective, the field of plasmonics has been in close relation with that of metamaterials. In fact, SPP resonances are responsible for achieving negative index of refraction in metallic metamaterials at optical wavelengths. ${ }^{18}$ As it will be shown, we also present that the internal SPPs that our model predicts on a double layer hole array play a fundamental role in achieving a negative effective permeability behavior as the electromagnetic (EM) field forms a virtual current loop between the two metallic layers. These plasmonic resonances are thus sometimes called $L C$ resonances ${ }^{19-22}$ and are used for achieving negative permeability in metamaterials such as the fishnet. ${ }^{19-23}$ The realization of metamaterials at optical wavelengths holds the promise for novel and exciting applications such as superlenses, ${ }^{24}$ hyperlenses, ${ }^{25}$ or cloaking devices. ${ }^{26}$

Finally, the influence of the alignment of the metallic hole arrays on EOT is also studied. In some configurations, the lateral displacement between the metallic hole arrays permits no direct line of sight through the structure. Nonetheless, the transmission remains remarkably large at resonance. The numerical results show the important role of the EM field in the transmission behavior of the SPP resonances. The structure presented here is of significant importance to the aforementioned applications, especially at optical and terahertz radiation, because smooth interfaces can be realized in the layered structures using modern growth techniques ${ }^{27}$ and has potential applications in subwavelength photolithography, nearfield microscopy, wavelength-tunable filters, optical modulators, solar selective surfaces, and directional radiation, among others.

The paper is structured as follows: in Sec. II the doublelayer metallic structure as well as the system used to analytically obtain the SPP dispersion relation is described. In Sec. III the numerical analysis of the double-layer metallic hole array structure is carried out. A discussion of the structure parameters' influence on the transmission is presented. Finally, some conclusions are given in Sec. IV.

\section{DESCRIPTION AND MODEL OF THE DOUBLE-LAYER METALLIC HOLE ARRAY}

In this work we consider the role of SPPs in the transmission through a dielectric layer sandwiched between two metallic hole arrays. In order to elucidate the general properties of SPPs on a double-layer metallic hole array, we can calculate the dispersion relation for the SPPs propagating on the multilayer geometry without holes depicted in Fig. 1. The desired dispersion relation has been previously calculated, ${ }^{7}$ but we extend it to the general case in which all the layers have an arbitrary electric permittivity $\varepsilon_{i}$.

Similarly to the case of a partially filled waveguide, the EM fields considered here are referred to as longitudinal section magnetic $\left(\mathrm{LSM}_{z}\right)$ where the subscript $z$ refers to the direction which is perpendicular to the interface. ${ }^{28}$ These hybrid modes are also named as $\mathrm{TM}_{z}$. The choice of this kind of EM modes is simply for convenience to satisfy the boundary conditions at the different interfaces. TE modes are not considered as it is widely known that for common media, SPPs only exist for TM polarization. ${ }^{29}$

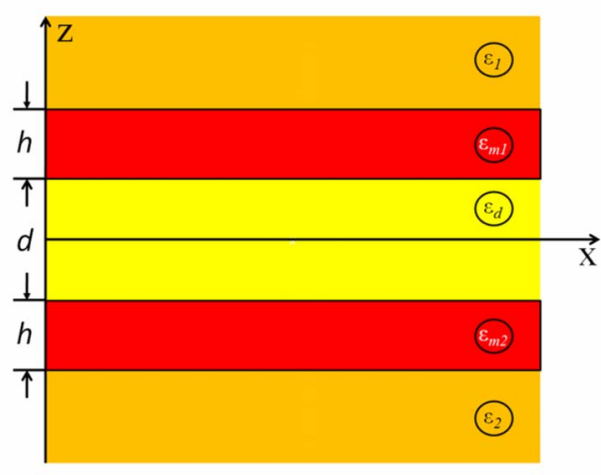

FIG. 1. (Color online) Geometry of a five-layer system formed by a dielectric film (thickness $d$, permittivity $\varepsilon_{d}$ ) sandwiched between two metal layers (thickness $h$, permittivity $\varepsilon_{m 1}$ and $\varepsilon_{m 2}$ ) and surrounded by two semi-infinite dielectric layers (permittivities $\varepsilon_{1}$ and $\left.\varepsilon_{2}\right)$.

In order to derive the expressions of the EM fields we begin by defining a proper expression for the magnetic and electric vector potentials called $\vec{A}$ and $\vec{F}$, respectively. It is known $^{28}$ that it is sufficient to let $\vec{A}$ have a unique component in the direction in which the fields are desired to be TM when deriving the field expressions that are TM to a given direction, independently of the coordinate system. The remaining components of $\vec{A}$ as well as all of $\vec{F}$ are set equal to zero.

As Fig. 1 shows, without loss of generality, we let the layers be parallel to the $x$ axis so for simplicity a onedimensional problem is assumed. Therefore $\varepsilon=\varepsilon(z)$ depends only on one spatial coordinate as the layers are considered infinite in the $y$ direction. To calculate the dispersion relation of the structure we consider the waves propagating along the $x$ direction of a Cartesian coordinate system, showing no spatial variation in the perpendicular, out-of-plane $y$ direction. Moreover we seek solutions with an evanescent decay in the $z$ direction normal to the interfaces, i.e., we look for propagating wave solutions confined to the interfaces. Having all this in mind, $\vec{A}$, which must satisfy the Helmholtz equation, can be described as $\vec{A}=A_{z} \hat{z}=A f(z) e^{i \beta x} \hat{z}$. The function $f(z)=e^{k_{z} z}$ describes the depth dependence of the EM fields. In general, $k_{z}= \pm \sqrt{\beta^{2}-k_{0}^{2} \varepsilon}$ with both plus and minus signs admissible. However, only one value of $k_{z}$ is allowed, corresponding to the solution that approaches zero when $z$ $\rightarrow \pm \infty . \beta$ is called the propagation constant of the traveling waves and corresponds to the component of the wave vector in the direction of propagation. Once $A_{z}$ is found, the next step is to find the EM field components via ${ }^{28}$

$$
\begin{gathered}
E_{x}=-j \frac{1}{\varpi \mu \varepsilon} \frac{\partial^{2} A_{z}}{\partial x \partial z}, \quad H_{x}=0, \\
E_{y}=0, \quad H_{y}=-\frac{1}{\mu} \frac{\partial A_{z}}{\partial x},
\end{gathered}
$$




$$
E_{z}=-j \frac{1}{\varpi \mu \varepsilon}\left(\frac{\partial^{2}}{\partial z^{2}}+k_{0}^{2} \varepsilon\right) A_{z}, \quad H_{z}=0,
$$

where the homogeneity in the $y$ direction $(\partial / \partial y=0)$ has been taken into account. Using the equation set (1)-(3) in all layers yields

$$
\begin{gathered}
H_{y}=-j \frac{\beta}{\mu} A e^{-k_{1} z} e^{j \beta x}, \\
E_{x}=-\frac{\beta k_{1}}{\varpi \mu \varepsilon_{0} \varepsilon_{1}} A e^{-k_{1} z} e^{j \beta x}, \\
E_{z}=-j \frac{\beta^{2}}{\varpi \mu \varepsilon_{0} \varepsilon_{1}} A e^{-k_{1} z} e^{j \beta x}
\end{gathered}
$$

for $z>d / 2+h$;

$$
\begin{gathered}
H_{y}=-j \frac{\beta}{\mu} B e^{-k_{2} z} e^{j \beta x}-j \frac{\beta}{\mu} C e^{k_{2} z} e^{j \beta x}, \\
E_{x}=-\frac{\beta k_{2}}{\varpi \mu \varepsilon_{0} \varepsilon_{m 1}} B e^{-k_{2} z} e^{j \beta x}+\frac{\beta k_{2}}{\varpi \mu \varepsilon_{0} \varepsilon_{m 1}} C e^{k_{2} z} e^{j \beta x}, \\
E_{z}=-j \frac{\beta^{2}}{\varpi \mu \varepsilon_{0} \varepsilon_{m 1}} B e^{-k_{2} z} e^{j \beta x}-j \frac{\beta^{2}}{\varpi \mu \varepsilon_{0} \varepsilon_{m 1}} C e^{k_{2} z} e^{j \beta x}
\end{gathered}
$$

for $d / 2<z<d / 2+h$

$$
\begin{gathered}
H_{y}=-j \frac{\beta}{\mu} D e^{-k_{3} z} e^{j \beta x}-j \frac{\beta}{\mu} E e^{k_{3} z} e^{j \beta x}, \\
E_{x}=-\frac{\beta k_{3}}{\varpi \mu \varepsilon_{0} \varepsilon_{d}} D e^{-k_{3} z} e^{j \beta x}+\frac{\beta k_{3}}{\varpi \mu \varepsilon_{0} \varepsilon_{d}} E e^{k_{3} z} e^{j \beta x},
\end{gathered}
$$

$$
E_{z}=-j \frac{\beta^{2}}{\varpi \mu \varepsilon_{0} \varepsilon_{d}} D e^{-k_{3} z} e^{j \beta x}-j \frac{\beta^{2}}{\varpi \mu \varepsilon_{0} \varepsilon_{d}} E e^{k_{3} z} e^{j \beta x}
$$

for $-d / 2<z<d / 2$;

$$
\begin{gathered}
H_{y}=-j \frac{\beta}{\mu} F e^{-k_{4} z} e^{j \beta x}-j \frac{\beta}{\mu} G e^{k_{4} z} e^{j \beta x}, \\
E_{x}=-\frac{\beta k_{4}}{\varpi \mu \varepsilon_{0} \varepsilon_{m 2}} F e^{-k_{4} z} e^{j \beta x}+\frac{\beta k_{4}}{\varpi \mu \varepsilon_{0} \varepsilon_{m 2}} G e^{k_{4} z} e^{j \beta x}, \\
E_{z}=-j \frac{\beta^{2}}{\varpi \mu \varepsilon_{0} \varepsilon_{m 2}} F e^{-k_{4} z} e^{j \beta x}-j \frac{\beta^{2}}{\varpi \mu \varepsilon_{0} \varepsilon_{m 2}} G e^{k_{4} z} e^{j \beta x}
\end{gathered}
$$

for $(-d / 2+h)<z<-d / 2$; and

$$
\begin{gathered}
H_{y}=-j \frac{\beta}{\mu} H e^{k_{5} z} e^{j \beta x}, \\
E_{x}=\frac{\beta k_{5}}{\varpi \mu \varepsilon_{0} \varepsilon_{2}} H e^{k_{5} z} e^{j \beta x}, \\
E_{z}=-j \frac{\beta^{2}}{\varpi \mu \varepsilon_{0} \varepsilon_{2}} H e^{k_{5} z} e^{j \beta x}
\end{gathered}
$$

for $z<-(d / 2+h)$, where $k_{i}=k_{z i}$ is the component of the wave vector perpendicular to the interface. In the central regions the modes localized at the interfaces couple due to the fact that the separation between the layers is small enough to allow coupling of the evanescent fields, as can be seen in the equations. Finally, the dispersion relation is obtained by solving the linear equation system enforcing the continuity of the tangential EM components at every interface separating two media which yields an implicit expression for $\beta$ and $\varpi$ via

$$
\begin{gathered}
\frac{\left(\frac{k_{2}}{\varepsilon_{m 1}}-\frac{k_{3}}{\varepsilon_{d}}\right)\left(\frac{k_{1}}{\varepsilon_{1}}+\frac{k_{2}}{\varepsilon_{m 1}}\right) e^{k_{2} h} e^{-k_{3} d / 2}+\left(\frac{k_{2}}{\varepsilon_{m 1}}+\frac{k_{3}}{\varepsilon_{d}}\right)\left(\frac{k_{1}}{\varepsilon_{1}}-\frac{k_{2}}{\varepsilon_{m 1}}\right) e^{-k_{2} h} e^{-k_{3} d / 2}}{\left.\left(\frac{k_{2}}{\varepsilon_{d}}\right) \frac{k_{3}}{\varepsilon_{1}}\right)\left(\frac{k_{1}}{\varepsilon_{1}}+\frac{k_{2}}{\varepsilon_{m}}\right) e^{k_{2} h} e^{k_{3} d / 2}+\left(\frac{k_{2}}{\varepsilon_{m 1}}-\frac{k_{3}}{\varepsilon_{d}}\right)\left(\frac{k_{1}}{\varepsilon_{1}}-\frac{k_{2}}{\varepsilon_{m 1}}\right) e^{-k_{2} h} e^{k_{3} d / 2}} \\
-\frac{\left(\frac{k_{4}}{\varepsilon_{m 2}}-\frac{k_{3}}{\varepsilon_{d}}\right)\left(\frac{k_{5}}{\varepsilon_{2}}-\frac{k_{4}}{\varepsilon_{m 2}}\right) e^{-k_{4} h} e^{k_{3} d / 2}+\left(\frac{k_{4}}{\varepsilon_{m 2}}+\frac{k_{3}}{\varepsilon_{d}}\right)\left(\frac{k_{4}}{\varepsilon_{m 2}}+\frac{k_{5}}{\varepsilon_{2}}\right) e^{k_{4} h} e^{k_{3} d / 2}}{\left(\frac{k_{4}}{\varepsilon_{m 2}}+\frac{k_{3}}{\varepsilon_{d}}\right)\left(\frac{k_{5}}{\varepsilon_{2}}-\frac{k_{4}}{\varepsilon_{m 2}}\right) e^{-k_{4} h} e^{-k_{3} d / 2}+\left(\frac{k_{4}}{\varepsilon_{m 2}}-\frac{k_{3}}{\varepsilon_{d}}\right)\left(\frac{k_{4}}{\varepsilon_{m 1}}+\frac{k_{5}}{\varepsilon_{2}}\right) e^{k_{4} h} e^{-k_{3} d / 2}}=0 .
\end{gathered}
$$

For the sake of simplicity, we limit ourselves to the symmetric case in which both the semi-infinite layers' relative dielectric constants are equal to $\varepsilon_{1}=\varepsilon_{2}=\varepsilon_{\text {air }}$ and $\varepsilon_{m 1}=\varepsilon_{m 2}$ $=\varepsilon_{\mathrm{Cr}}$, where $\mathrm{Cr}$ stands for chromium modeled with a Drude dispersion. ${ }^{30}$ The results presented here are easily extensible for other types of metal.
The SPPs dispersion relation $\beta(\varpi)$ given by Eq. (9) accounts for two types of SPP modes decoupled from each other, namely, the internal SPP and the external SPP which propagate along the internal and the external metal-dielectric interfaces, respectively. ${ }^{7}$

For our modeling of double layer hole arrays, we take 


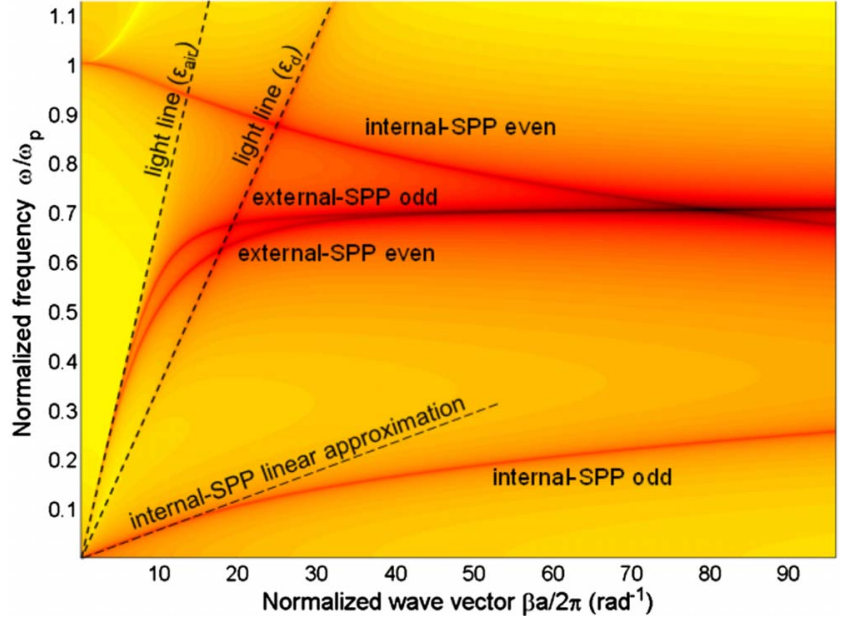

FIG. 2. (Color online) Dispersion relation of SPPs at a dielectric ( $d=4 \mathrm{~nm}$ and $\left.\varepsilon_{d}=4\right)$ sandwiched between Drude double-metal layers $(h=45 \mathrm{~nm})$ surrounded by air.

Eq. (9) as a starting point, as it describes the fundamentals of SPPs in smooth double-layer metallic structure interfaces. However, in analogy to what is done for single-layer structures, we can consider that the SPP dispersion relation on a double-layer hole array will be similar to that obtained in the smooth double-layer metallic structure. This procedure allows us to predict analytically all the EOT peaks coming from the excitation of both internal- and external-SPP modes at those wavelengths at which they couple to the incoming light via grating coupling on a double-layer hole array structure. However, this model is clearly an approximation, since it does not take into account the effect of the holes on the SPP dispersion relation.

In a metal film with a periodic square array of holes, the interaction between light and the SPP obeys momentum and energy conservation given by ${ }^{2}$

$$
\left|\vec{k}_{s p p}\right|=\left|\vec{k}_{x}+\vec{G}_{i, j}\right|=\left|\vec{k}_{0} \sin \phi+i \vec{G}_{x}+j \vec{G}_{y}\right|,
$$

where $\left|\vec{k}_{s p p}\right| \equiv \beta(\varpi)$ is the wave vector of the SPP derived from Eq. (9), $\vec{k}_{0} \sin \phi$ is the in-plane component of the incident wave vector, $\vec{G}_{x}$ and $\vec{G}_{y}$ are the reciprocal lattice vectors where for a square array have the same value $\left|\vec{G}_{x}\right|=\left|\vec{G}_{y}\right|$ $=2 \pi / a, a$ being the lattice periodicity and $i, j$ are both integers.

The dispersion relation given by Eq. (9), with $h=45 \mathrm{~nm}$, $d=4 \mathrm{~nm}$ and $\varepsilon_{d}=4$, is represented in Fig. 2 in a purely real $\beta-\varpi$ plane, thus neglecting the imaginary part of the solution which does not affect our modeling of the resonant frequencies. The internal and external-SPPs dispersion curves can be seen, with odd and even symmetric modal fields with respect to the $x$ axis due to the symmetry of the structure. ${ }^{6,7,29}$ The light lines are trivial solutions for our problem and correspond to the case when all fields are zero. ${ }^{7}$

For large wave vectors the frequency of the SPP tends to the characteristic SPP frequency ${ }^{29}$

$$
\varpi_{s p}=\frac{\varpi_{p}}{\sqrt{\varepsilon_{\infty}+\varepsilon_{i}}},
$$

with $\varepsilon_{i}=\varepsilon_{d}$ or $\varepsilon_{\text {air }}$ for internal or external SPPs, respectively. This trend supports the interpretation of internal and external SPP modes. In the opposite regime of short wave vectors $\beta$ $\ll k_{p}$, corresponding to low frequencies, the SPP propagation constant related to internal SPPs can be described according to the linear approximation

$$
\beta^{\text {int }} \equiv\left|\vec{k}_{s p}^{\text {int }}\right|=\left|\vec{k}_{o}\right| \sqrt{\varepsilon_{d}}\left[\frac{d}{d+\lambda_{p} \operatorname{coth}\left(k_{p} h\right) / \pi}\right]^{-1 / 2},
$$

which is an extension of that presented in Ref. 7, where $k_{p}$ $=2 \pi / \lambda_{p}=\varpi_{p} / c$. Regarding the external SPP curves, for the same frequency range, they can be described by

$$
\beta^{\mathrm{ext}} \equiv\left|\vec{k}_{s p}^{\mathrm{ext}}\right|=\left|\vec{k}_{o}\right| \sqrt{\frac{\varepsilon_{\mathrm{air}} \varepsilon_{m}}{\varepsilon_{\mathrm{air}}+\varepsilon_{m}}},
$$

where it has been considered that in this frequency range the odd and even external SPPs are close enough to be represented by the same expression.

It can be seen that, as the dielectric thickness $d$ decreases, the internal-SPP mode lowers in frequency due to an increased coupling between the SPPs at the two internal interfaces. On the other hand, as it is expected, the external SPP is very close to the light line of the cladding, i.e., $\beta^{\text {ext }} \approx\left|\vec{k}_{o}\right|$, which does not vary with $d$.

The straightforward analytical expressions (12) and (13) can be substituted into Eq. (10) to analytically predict the approximate excitation frequencies at which the incoming light couples to both the internal and external SPPs on a double layer hole array via grating coupling. As can be seen, when applying Eq. (10), several modes $(i, j)$ will appear for both internal and external SPPs, accounting for all the EOT peaks arising from SPPs in the double-layer hole array. Indeed, this model permits a complete identification, classification, and enumeration of such peaks.

\section{NUMERICAL RESULTS}

Once the dispersion relation is known, we perform numerical simulations by using the commercial software CST Microwave Studio, a general-purpose EM simulator based on the finite integration technique (FIT), to investigate the transmission through the double-metallic layers and its dependence with the parameters of the sandwiched dielectric. As depicted in Fig. 3 the structure lies on the $x y$ plane and consists of a dielectric layer of variable thickness $d$, and permittivity $\varepsilon_{d}$, sandwiched between two chromium metal layers of thickness $h$ nanostructured with subwavelength circular hole arrays filled with air. The surrounding medium is also considered to be air. The incoming wave is considered to be normal to the structure with the $\vec{E}$ field polarized along the $x$ axis. The metal layers' parameters $\varepsilon_{\infty}=1, \varpi_{p}=6.7034$ $\times 10^{15} \mathrm{rad} / \mathrm{s}$, and $\gamma=1.138 \times 10^{13} \mathrm{~Hz}$ for the Drude model are extracted from Ref. 31. The thickness of the metal $h$ $=100 \mathrm{~nm}$, the radius of the hole $r=800 \mathrm{~nm}$, and the lattice parameter $a=3990 \mathrm{~nm}$ are kept constant in all the simula- 


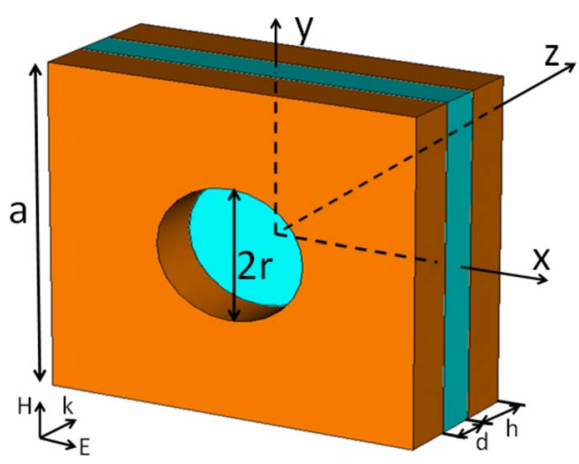

FIG. 3. (Color online) Schematic diagram of the double-metallic layer hole array unit cell. The unit cell structural parameters are labeled: period $a$, hole radius $r$, metal thickness $h$, and dielectric separation $d$.

tions. The dielectric thickness $d$ and permittivity $\varepsilon_{d}$ will be varied throughout the study. Unit cell boundary conditions are applied for in-plane boundaries representing an infiniteperiodic structure, and open boundaries are considered in the perpendicular direction.

The normalized transmission spectrum together with the dispersion relation given by Eq. (9) for a dielectric thickness $d=300 \mathrm{~nm}$ is depicted in Fig. 4 for both (a) $\varepsilon_{d}=1$ and (b) $\varepsilon_{d}=4$. The predicted EOT peak frequencies calculated by equating the matching condition given by Eq. (10) with the full dispersion relation of Eq. (9) (solid lines) are shown for different values of $(i, j)$ of both internal and external SPPs. The predicted frequencies are seen to approximately match the simulated spectra EOT peaks (dashed lines). Agreement between simulation and our model is not perfect, in the same way as in a single-layer hole array, because the dispersion relation of the SPPs used as matching condition does not take into account the presence of the holes, which cause scattering losses and a resonance shift. As a consequence, the predicted resonant frequencies are slightly larger than those obtained in simulations. ${ }^{4}$ Although not indicated, the multiple small peaks seen in Fig. 4 at high frequencies can each be accurately associated with a certain $(i, j)$ resonance of either internal or external SPPs.

On the other hand, the transmission minima are the result of Wood's anomaly. ${ }^{32}$ The dotted lines in Fig. 4 highlight the position of Wood's anomalies for different $(i, j)$. It can be seen how the expected Wood's anomalies positions are in a good agreement with the minima positions of the simulated spectra.

We will now analyze in detail the features of the EM fields in each peak and confirm their agreement with the model. We will start by analyzing the external-SPP resonances. Two main peaks for the external SPP appear in the simulated frequency range, which correspond to the first- and second-order transmission resonances according to Eqs. (10) and $(13)$ when $(i, j)=(1,0)$ and $(1,1)$, respectively. Note that, when $\varepsilon_{d}=4$, the simulated spectra show two distinct separate peaks corresponding to the odd and even symmetric $(1,0)$ external-SPP modes, while the dispersion relation used by the model predicts a single hybridized frequency for both. This is because the external SPPs on opposite interfaces experience increased coupling through the holes, which the
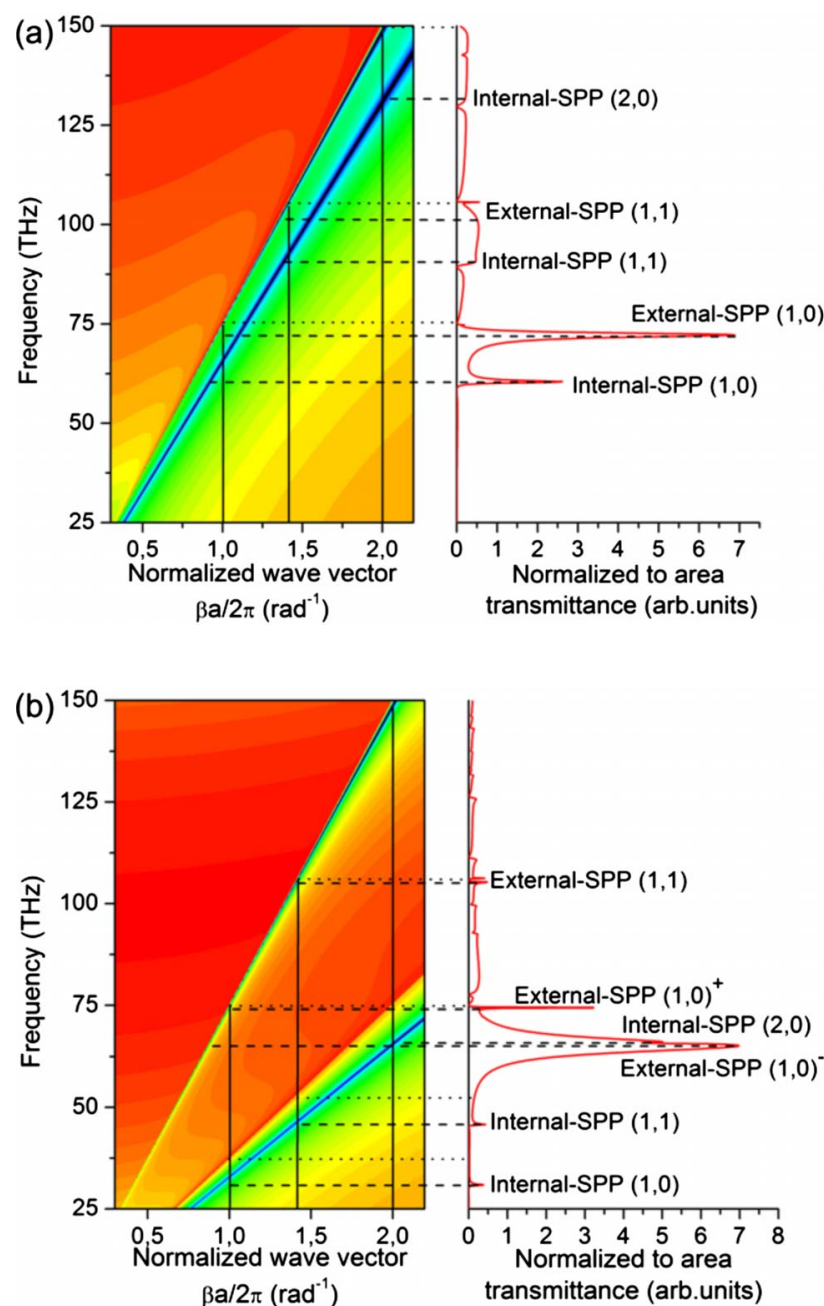

FIG. 4. (Color online) Representation of the dispersion relation (left) and the calculated normalized transmission (right) for (a) $\varepsilon_{d}$ $=1$ and (b) $\varepsilon_{d}=4$. In both cases $d=300 \mathrm{~nm}$. The solid lines represent the position at which the matching grating conditions are fulfilled. The simulated EOT peak frequencies are shown with dashed lines. The dotted lines highlight the expected position of Wood's anomalies. The subscripts + and - stand for odd and even symmetry modes, respectively.

model does not take into account. Figure 5 shows the mode profiles of the first-order external-SPP resonances for both $\varepsilon_{d}=1$ and 4 when $d=300 \mathrm{~nm}$. It can be seen that the external-SPP EM fields are located on the outer metal-air interfaces, being particularly concentrated at the edge of the holes. Also note that for the case $\varepsilon_{d}=4$, the $(1,0)$ external resonances are very close in frequency to an internal resonance, which explains why SPPs can also be seen in the inner dielectric layer.

We now analyze the internal-SPP resonances. Several EOT peaks owing to internal SPPs are seen and indicated in Fig. 4. The EM field profiles for the first and second internalSPP modes are plotted in Fig. 6, both for $\varepsilon_{d}=1$ and 4 . These EM field patterns fully match our predictions. The EM fields are clearly concentrated on the internal dielectric layer, as expected for an internal-SPP resonance. Also, the different modes $(i, j)$ show the expected wavelike features in the transversal direction. 


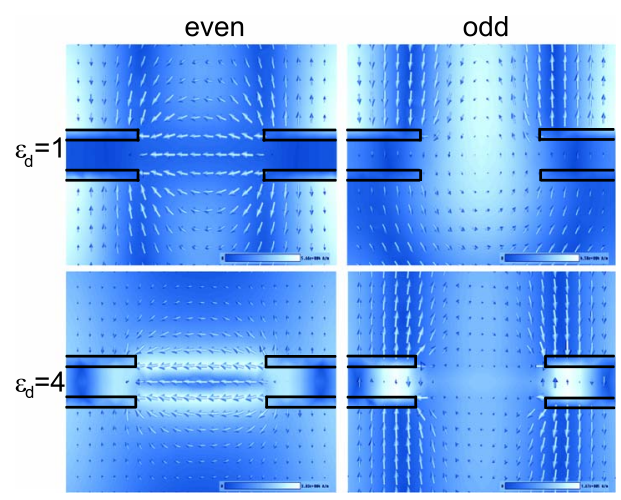

FIG. 5. (Color online) EM field distribution for even (left) and odd (right) external-SPP resonance at $x z$ plane for $d=300 \mathrm{~nm}$ and $\varepsilon_{d}=1$ (top) and $\varepsilon_{d}=4$ (down). The arrows represent the $E$ field; the color scale represents $|H|$ and points out of the page. Blue (gray) and white colors display zero and maximum values of the amplitude of the magnetic field, respectively. The black lines are a guide for the eyes enclosing the metallic layers.

The internal SPPs show in general less transmission than the external SPPs as they are concentrated inside optically thick metallic layers which increase the attenuation due to the absorption of the metal layer. The external SPPs peaks show high transmission, as the EM field is concentrated at the edges of the holes and the transmission is achieved by tunneling through them. . $^{3,33}$

As intuitively expected, the inner dielectric permittivity affects the internal-SPP dispersion relation while the outer dielectric determines the external-SPP dispersion relation. By

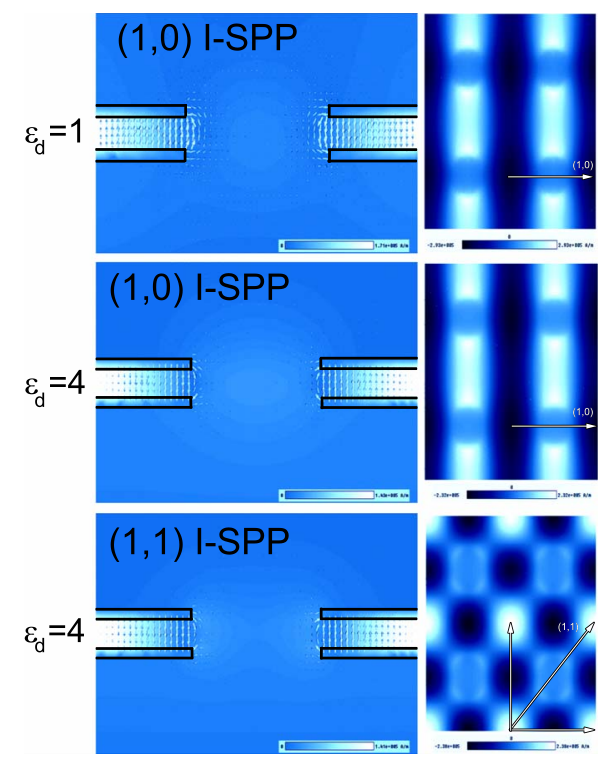

FIG. 6. (Color online) EM field distribution at $(1,0)$ (top and middle) and $(1,1)$ (down) internal-SPP resonance for $d=300 \mathrm{~nm}$ and $\varepsilon_{d}=1$ (top) and $\varepsilon_{d}=4$ (middle and down). (Left) The $E$ field is represented by arrows; the color scale represents $|H|$ at $x z$ plane. The black lines are a guide for the eyes enclosing the metallic layers. (Right) $H_{y}$ distribution maps in the $x y$ plane. Dark blue (black), blue (gray), and white colors display minimum, zero, and maximum values of the amplitude of the magnetic field, respectively. increasing the inner dielectric permittivity, the dispersion relation of the internal SPP shifts down in frequency, reducing its slope by a factor $\varepsilon_{d}^{1 / 2}$. The external SPP is unaffected by the inner dielectric, except for some considerations which we study later. This behavior is in good agreement with simulations.

Moreover, the dielectric permittivities can be adjusted to excite the external- and internal-SPP modes of different orders $(i, j)$ at the same frequency overlapping its transmission maxima, as it is almost the case for the even external $(1,0)$ and internal $(2,0)$ SPP modes when $\varepsilon_{d}=4$ [see Fig. 4(b)], thus forming a single peak in which the resulting EM field has a $(2,0)$ pattern in the inner dielectric layer and a $(1,0)$ pattern in the claddings.

Also it should be noticed how the transmission for both the internal and external SPPs decreases when the inner dielectric permittivity $\varepsilon_{d}$ differs from that of the surrounding claddings. This behavior is similar to that of a single metal layer when the claddings surrounding the metal are different, so that decreased coupling of the SPP sustained in each metal-dielectric interface is allowed giving rise to a decrease in transmission. ${ }^{16,33,34}$

Taking into account the subwavelength thickness of the structure, we retrieved from the transmitted and reflected spectra the effective constituent parameters of the structure ${ }^{35}$ shown in Fig. 7 for the cases when dielectric permittivity is $\varepsilon_{d}=1$ and 4 and $d=300 \mathrm{~nm}$. It can be seen that the retrieved $\varepsilon_{\text {eff }}$ is negative below the first external-SPP EOT peak, while $\mu_{\text {eff }}$ becomes negative at the internal-SPP resonances. This magnetic negative response can be explained looking at the current and field distribution for the internal-SPP modes. Antiparallel currents are excited at opposite internal metallic interfaces, closed by an electric displacement current. Thus, a virtual current loop (VCL) between the metallic layers on a perpendicular plane to the incoming magnetic field will be formed giving rise to a magnetic resonant response of negative $\mu_{\text {eff. }}{ }^{36}$ If the internal-SPP resonances occur below the external-SPP resonance, negative $\varepsilon_{\text {eff }}$ and $\mu_{\text {eff }}$ coincide in frequency and a negative effective index $n_{\text {eff }}$ is retrieved, thus, achieving an EOT with a negative effective index nature. ${ }^{37}$ This was indeed the case with our simulations. The internal-SPP resonances have been sometimes called $L C$ resonances due to their analogy to a $L C$ circuit. ${ }^{19-22}$ The presented dispersion relation of internal SPPs is consistent with the $L C$ model: an increase in $\varepsilon_{d}$ lowers the resonant frequency, while an increase in $d$ increases the resonant frequency. From the internal-SPP dispersion curve Eq. (12) and the matching condition Eq. (10) we can derive an expression, similar to that presented in Ref. 20, linking the resonant frequency at which an internal SPP is excited with the frequency $\varpi_{L C}$ of the $L C$ equivalent circuit of the VCL,

$$
\lambda_{i, j}^{\mathrm{int}}=\frac{2 \pi}{\left|\vec{G}_{i, j}\right|} \sqrt{\varepsilon_{d}}\left[\frac{d}{d+\lambda_{p} \operatorname{coth}\left(k_{p} h\right) / \pi}\right]^{-1 / 2}=\frac{2 \pi c}{\left|\vec{G}_{i, j}\right|}\left(\frac{1}{\varpi_{L C}}\right) .
$$

Regarding the extracted parameters, the external-SPPs resonances do not show magnetic response because the displacement current does not form a VCL with the electric current 


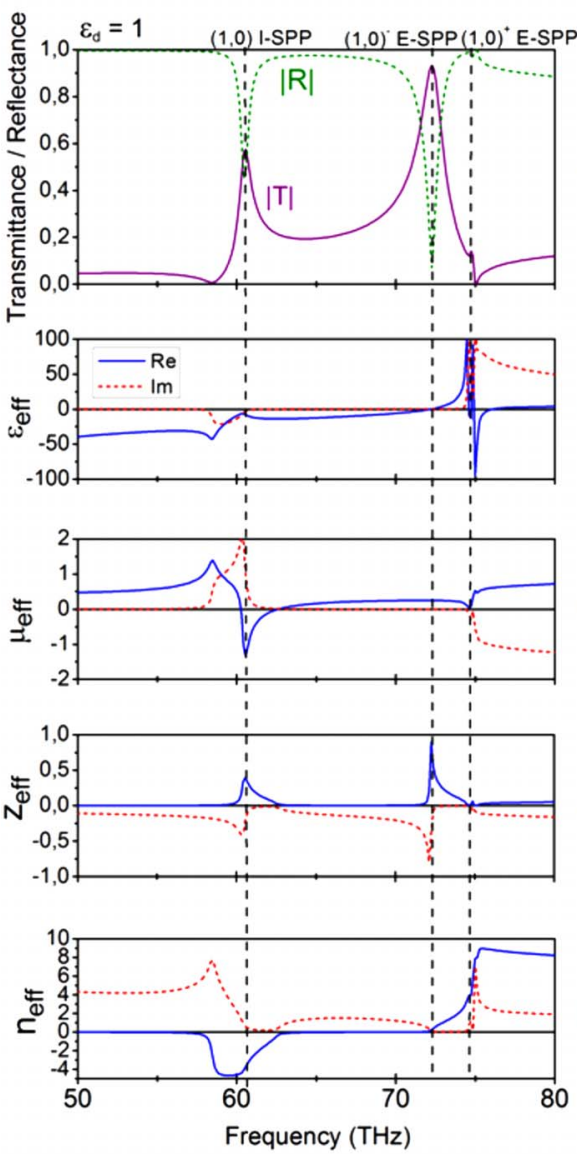

(a)

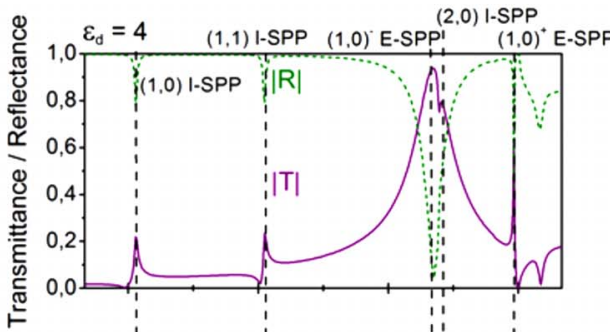

(c)

(b)

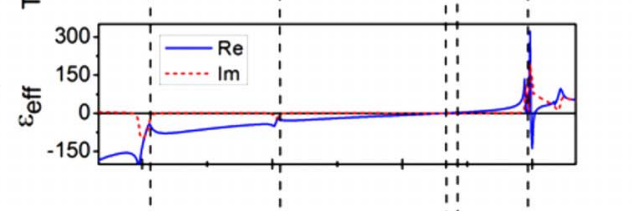

(d)

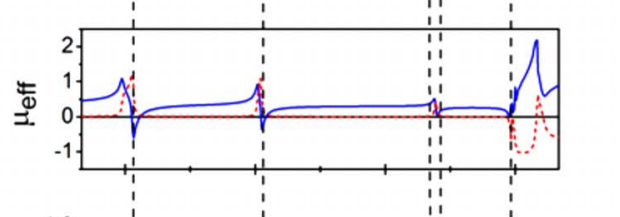

(e)

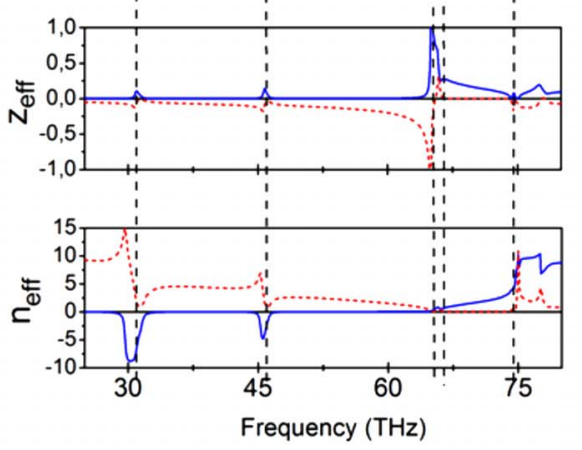

FIG. 7. (Color online) Transmission and reflection spectrum (a) and retrieved parameters of the structure: effective values of permittivity $\varepsilon_{\text {eff }}$, permeability $\mu_{\text {eff }}$, impedance $Z_{\text {eff }}$, and refractive index $n_{\text {eff }}$ are depicted in (b), (c), (d), and (e), respectively, for $\varepsilon_{d}$ $=1$ (left) and $\varepsilon_{d}=4$ (right). The dashed lines indicate the position of the resonant frequencies of the internal-SPP (I-SPP) and externalSPP (E-SPP) for different $(i, j)$ values. as the $E$ field is not concentrated between the plates but at the rims of the holes (see Fig. 5), rather their effect is to change the negative $\varepsilon_{\text {eff }}$ typical for metallic composites at low frequencies into a positive $\varepsilon_{\text {eff }}$, as required by the matching condition between $\varepsilon_{\text {eff }}$ and $\mu_{\text {eff }}\left(Z_{\text {eff }} \approx 1\right)$ for a high transmission peak, where $\mu_{\text {eff }}$ is known to be small and positive. However, for the antisymmetric external SPP a magnetic response is excited due to the fact that considerable $E_{z}$ remains between the metallic layers as to build the VCL. ${ }^{21}$

The presented relationship between the internal-SPP EOT peaks and its resonant negative magnetic response $\mu_{\text {eff }}$ in double-layer hole array is, in fact, a very appropriate way of modeling the well-known fishnet metamaterial whose structure is fundamentally the same as that presented here. ${ }^{19-23}$ In fact, in previous results the resonant magnetic response of the fishnet structure was simply termed a magnetic plasmon polariton (MPP) $)^{20-22}$ without further indication of its real physical origin. We now give this resonance a complete interpretation as the $(1,0)$ internal-SPP mode. The same is true for higher order internal-SPPs modes usually termed high order MPPs, ${ }^{20,21}$ which with the presented model can be clearly identified and enumerated.

Now we will study the effect of varying the dielectric thicknesses $d$ as shown in Fig. 8. The transmission for the external SPP decreases slightly as $d$ grows. ${ }^{11}$ On the other hand, the transmission of the internal SPP rises with $d$. As predicted by the model [Eqs. (12) and (13)] and confirmed by simulations [see Fig. 8(a)], a change in the inner dielectric thickness shifts the internal-SPP resonance while not affect- ing the external SPP, as it was previously stated. However, as shown in Fig. 8(b), the external SPP shifts in frequency for varying $d$ if $\varepsilon_{d}$ is different to that of the claddings. This external-SPP frequency shift with $d$ is not predicted by our model, as it considers smooth metal layers without holes, such that the external SPPs propagate entirely in the outer metal-air interfaces and so do not depend on the inner dielectric parameters. However, if one looks at the simulated EM field distribution of the external SPPs on the real hole array (see Fig. 5), it can be seen that the EM field is concentrated at the edges of the holes, and so the external SPPs do interact with the internal dielectric medium, due to its lack of holes, leading to a downshift of their resonant frequency. The interaction is greater for thicker internal dielectric layers. Nevertheless, we can adjust the presented model taking this into account by using an appropriately chosen equivalentpermittivity $\varepsilon_{\text {eq }}$, instead of the permittivity of the claddings, $\varepsilon_{\text {air }}$, so that Eq. (13) matches the simulated EOT frequencies. In this way, the interaction of the external SPPs with the inner dielectric due to the presence of the holes is being introduced in our hole-free model as an equivalent outer dielectric. ${ }^{16}$ Thus, $\varepsilon_{\text {eq }}$ will depend both on the external air and the internal dielectric at the holes, equaling $\varepsilon_{\text {air }}$ in the case of $d=0$, and increasing toward an average value between the cladding and dielectric permittivities as $d$ increases, as shown in Fig. 9. In that way, if $\varepsilon_{d}=1, \varepsilon_{\mathrm{eq}}$ remains constant and close to $\varepsilon_{\text {air }}$, so no resonant frequency variation is observed with $d$.

Up until now we have considered the hole arrays drilled only in the metal layers with a lack of holes in the inner 
(a)
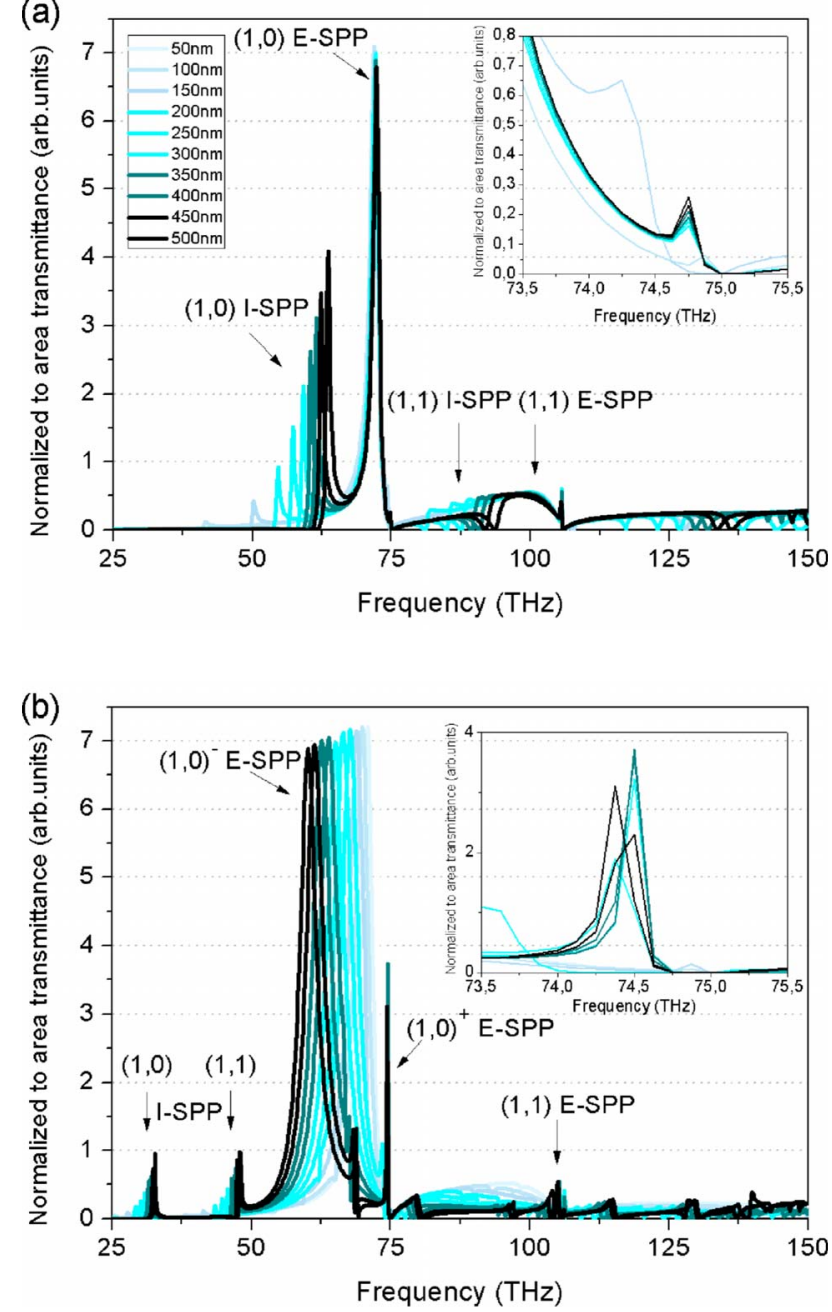

FIG. 8. (Color online) Normalized-to-area transmittance for different dielectric thickness $d$ and dielectric permittivity $\varepsilon_{d}$. (a) $\varepsilon_{d}$ $=1$ and (b) $\varepsilon_{d}=4$. The insets of both figures show the odd externalSPP resonance. The arrows mark the position of the internal-SPP (I-SPP) and external-SPP (E-SPP) for different resonances $(i, j)$.

dielectric. However, in the case that the dielectric layer had holes aligned with those in the metal layers, then the previously discussed external-SPP resonance shift with $d$ does not take place (not shown), suggesting that $\varepsilon_{\text {eq }}$ remains equal to $\varepsilon_{\text {air }}$ when holes are milled in the dielectric. The line of circles of Fig. 9 shows this case when $\varepsilon_{d}=4$. This supports the interpretation that the frequency dependence of the external SPPs with the inner dielectric arises due to the external-SPP EM fields entering the inner layers through the holes. Taking all this into account, we conclude that the suitable value for the equivalent-permittivity $\varepsilon_{\text {eq }}$, which depends on the thickness $d$ and dielectric permittivity $\varepsilon_{d}$, is highly determined by the structure design and its related EM field distribution. This behavior could be a means of tuning the external EOT peaks.

Finally, the influence of the alignment between the holes on the spectrum transmission is also studied. The metallic layers were displaced with respect to each other a distance of $a / m$, with $m$ ranging from 1 (perfect alignment) to 6 , along the reciprocal directions $\Gamma X$ and $\Gamma M$ of the reduced Brillouin zone, though only the transmission spectrums achieved with

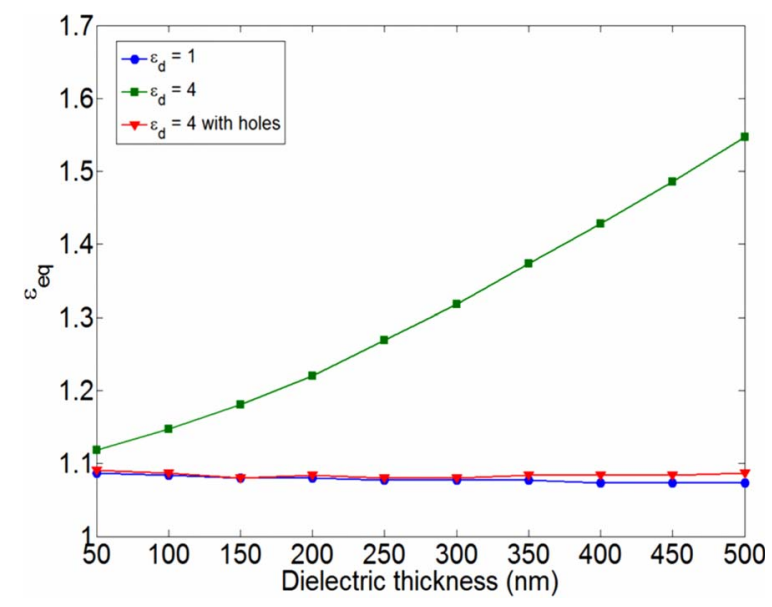

FIG. 9. (Color online) Variation of $\varepsilon_{\mathrm{eq}}$ vs $d$ for different $\varepsilon_{d}$ values.

$m=2,3$, and 4 are depicted in Fig. 10. We will study the case of $d=300 \mathrm{~nm}$ and $\varepsilon_{d}=1$. We find that the transmission, which depends strongly on the alignment of the holes, remains remarkably large at resonance, even though in some configurations the displacement of the two metal layers permits no direct line of sight through the structure. Moreover, the internal-SPP resonances, not only maintain, but rather increase their transmission compared with the aligned case and exceed, in most of the cases, the transmission of the external-SPP resonances, which show reduced transmission than in the aligned case. When the lateral shift between the two layers is close to half the grating period $(m=2)$ a maximum transmission is reached for the internal SPPs, further decreasing with $m$. This has been explained by the matching of the Poynting vector between the layers. ${ }^{10}$ However, we have noticed that the conclusions of Ref. 10 apply only to the internal SPPs but not to the external SPPs, due to the low confinement of the EM field between the layers, so the amount of transmission for the external SPPs is practically unaffected with the lateral displacement. Also, two additional

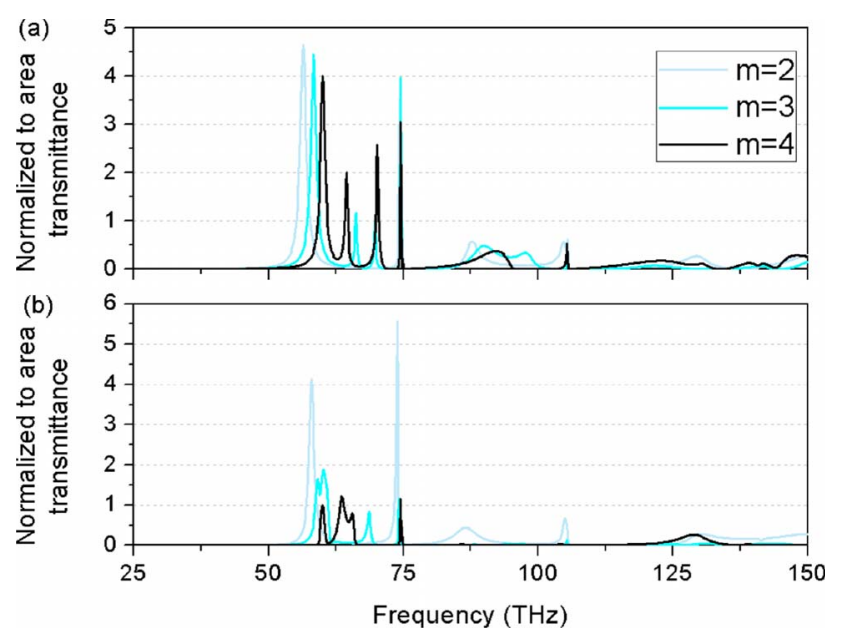

FIG. 10. (Color online) Transmission for samples with lateral shifts of $a / 2$ [light blue (light gray)], $a / 3$ [blue (gray)], and $a / 4$ (black) respectively, between the two metal layers for $d=300 \mathrm{~nm}$ and $\varepsilon_{d}=1$ for $\Gamma X$ (a) and $\Gamma M$ (b) directions. 
peaks appear in the spectrum. ${ }^{10}$ These peaks, likewise the ordinary EOT peaks, do not arise from neither Fabry-Perot resonances between two metallic layers, because the dielectric thickness embedded in the metal layers is much smaller than the wavelength of resonance, nor from propagating diffractive orders emerging from the metal layers by the normally incident light, because the period of the grating is smaller than the wavelength resonance. ${ }^{14}$

\section{CONCLUSIONS}

We have obtained analytically the dispersion relation of SPPs in a double-layer metallic structure and shown by numerical analysis that light coupling to the external and internal SPPs originates EOT. A good matching between the theoretical and simulated resonant frequencies is observed. Moreover, the strong influence of the sandwiched dielectric layer parameters in the transmission resonances is presented. These effects can be used to tune the resonant frequencies for both the internal and external SPPs. In addition, at the internal-SPP resonant frequencies a negative effective permeability is achieved as a VCL is formed between the me- tallic layers which can be used to design negative-index metamaterials. The influence of the alignment between holes was also studied. Even in the case of no direct line of sight through the structure, the transmission remained remarkably large at resonances. The model presented here surely facilitates the interpretation and design of double-layer structures.

The transmission features through double-layer subwavelength metallic hole arrays open up a new dimension in the design and operation of plasmonics devices. Understanding the coupling of evanescent waves in complex double-layer metallic hole arrays nanostructures is of fundamental interest and practical importance in designing optical devices that could become important building blocks in future nanooptical systems.

\section{ACKNOWLEDGMENTS}

Financial support by the Spanish MCyT and EU-FEDER under Contract No. TEC2005-06923-C03-03 is gratefully acknowledged. R.O., C.G.-M., and F.J.R.-F. also acknowledge financial support from grants FPI of Universidad Politécnica de Valencia, FPU of MICINN and from a grant of La Caixa, respectively. *ruormo@ntc.upv.es
${ }^{1}$ R. H. Ritchie, Phys. Rev. 106, 874 (1957).

${ }^{2}$ T. W. Ebbesen, H. J. Lezec, H. F. Ghaemi, T. Thio, and P. A. Wolf, Nature (London) 391, 667 (1998).

${ }^{3}$ L. Martín-Moreno, F. J. García-Vidal, H. J. Lezec, K. M. Pellerin, T. Thio, J. B. Pendry, and T. W. Ebbesen, Phys. Rev. Lett. 86, 1114 (2001).

${ }^{4}$ C. Genet and T. W. Ebbesen, Nature (London) 445, 39 (2007).

${ }^{5}$ D. E. Grupp, H. J. Lezec, T. W. Ebbesen, K. M. Pellerin, and T. Thio, Appl. Phys. Lett. 77, 1569 (2000); T. Thio, H. F. Ghaemi, H. J. Lezec, P. A. Wolff, and T. W. Ebbesen, J. Opt. Soc. Am. B 16, 1743 (1999); A. Degiron, H. J. Lezec, W. Barnes, and T. W. Ebbesen, Appl. Phys. Lett. 81, 4327 (2002); H. F. Ghaemi, T. Thio, D. E. Grupp, T. W. Ebbesen, and H. J. Lezec, Phys. Rev. B 58, 6779 (1998).

${ }^{6}$ H. Raether, Surface Plasmons on Smooth and Rough Surfaces and on Gratings, Springer Tracts in Modern Physics Vol. 111 (Springer-Verlag, Berlin, 1988).

${ }^{7}$ E. N. Economou, Phys. Rev. 182, 539 (1969).

${ }^{8}$ G. J. Kovacs and G. D. Scott, Phys. Rev. B 16, 1297 (1977).

${ }^{9}$ Y. Wang, Appl. Phys. Lett. 82, 4385 (2003).

${ }^{10}$ F. Miyamaru and M. Hangyo, Phys. Rev. B 71, 165408 (2005).

${ }^{11}$ Y. H. Ye and J. Y. Zhang, Opt. Lett. 30, 1521 (2005).

${ }^{12}$ H. Li, S. Xie, R. Zhou, Q. Liu, X. Zhou, and M. Yuan, J. Phys.: Condens. Matter 20, 415223 (2008).

${ }^{13}$ A. P. Hibbins, J. R. Sambles, C. R. Lawrence, and J. R. Brown, Phys. Rev. Lett. 92, 143904 (2004).

${ }^{14}$ H. B. Chan, Z. Marcet, K. Woo, D. B. Tanner, D. W. Carr, J. E. Bower, R. A. Cirelli, E. Ferry, F. Klemens, J. Miner, C. S. Pai, and J. A. Taylor, Opt. Lett. 31, 516 (2006).

${ }^{15}$ R. M. Bakker, V. P. Drachev, H. K. Yuan, and V. M. Shalaev, Opt. Express 12, 3701 (2004).
${ }^{16}$ Z. H. Tang, R. W. Peng, Z. Wang, X. Wu, Y. J. Bao, Q. J. Wang, Z. J. Zhang, W. H. Sun, and M. Wang, Phys. Rev. B 76, 195405 (2007).

${ }^{17}$ M. Beruete, M. Sorolla, and I. Campillo, Opt. Express 14, 5445 (2006); M. Beruete, M. Sorolla, M. Navarro-Cía, F. Falcone, I. Campillo, and V. Lomakin, ibid. 15, 1107 (2007).

${ }^{18}$ V. M. Shalaev, Nat. Photonics 1, 41 (2007); C. García-Meca, R. Ortuño, R. Salvador, A. Martínez, and J. Martí, Opt. Express 15, 9320 (2007); F. J. Rodriguez-Fortuño, C. García-Meca, R. Ortuño, J. Martí, and A. Martínez, Phys. Rev. B 79, 075103 (2009).

${ }^{19}$ S. Zhang, W. Fan, N. C. Panoiu, K. J. Malloy, R. M. Osgood, and S. R. J. Brueck, Phys. Rev. Lett. 95, 137404 (2005).

${ }^{20}$ T. Li, J. Q. Li, F. M. Wang, Q. J. Wang, H. Liu, S. N. Zhu, and Y. Y. Zhu, Appl. Phys. Lett. 90, 251112 (2007).

${ }^{21}$ T. Li, H. Liu, F. M. Wang, J. Q. Li, Y. Y. Zhu, and S. N. Zhu, Phys. Rev. E 76, 016606 (2007).

${ }^{22}$ T. Li, H. Liu, F. M. Wang, Z. G. Dong, S. N. Zhu, and X. Zhang, Opt. Express 14, 11155 (2006).

${ }^{23}$ S. Zhang, W. Fan, K. J. Malloy, S. R. J. Brueck, N. C. Panoiu, and R. M. Osgood, Opt. Express 13, 4922 (2005); G. Dolling, C. Enkrich, M. Wegener, C. M. Soukoulis, and S. Linden, Opt. Lett. 31, 1800 (2006); G. Dolling, C. Enkrich, M. Wegener, C. M. Soukoulis, and S. Linden, Science 312, 892 (2006).

${ }^{24}$ J. B. Pendry, Phys. Rev. Lett. 85, 3966 (2000); N. Fang, H. Lee, C. Sun, and X. Zhang, Science 308, 534 (2005); D. R. Smith, ibid. 308, 502 (2005).

${ }^{25}$ N. Fang, H. Lee, C. Sun, and X. Zhang, Science 308, 534 (2005); Z. Liu, H. Lee, Y. Xiong, C. Sun, and X. Zhang, ibid. 315, 1686 (2007).

${ }^{26}$ J. B. Pendry, D. Schurig, and D. R. Smith, Science 312, 1780 (2006); W. Cai, U. K. Chettiar, A. V. Kildishev, and V. M. 
Shalaev, Nat. Photonics 1, 224 (2007).

${ }^{27}$ G. Dehlinger, L. Diehl, U. Gennser, H. Sigg, J. Faist, K. Ensslin, D. Grützmacher, and E. Müller, Science 290, 2277 (2000).

${ }^{28}$ C. A. Balanis, Advanced Engineering Electromagnetics (Wiley, New York, 1989).

${ }^{29}$ S. A. Maier, Plasmonics: Fundamental and Applications (Springer, New York, 2007).

${ }^{30}$ P. Drude, Ann. Phys. 306, 566 (1900).

${ }^{31}$ A. D. Rakic, A. B. Djurisic, J. M. Elazar, and M. L. Majewski, Appl. Opt. 37, 5271 (1998).

${ }^{32}$ R. W. Wood, Philos. Mag. 4, 396 (1902); R. W. Wood, Phys. Rev. 48, 928 (1935).
${ }^{33}$ S. A. Darmanyan and A. V. Zayats, Phys. Rev. B 67, 035424 (2003).

${ }^{34}$ A. Krishnan, T. Thio, T. J. Kim, H. J. Lezec, T. W. Ebbesen, P. A. Wolff, J. B. Pendry, L. Martín-Moreno, and F. J. Garcia-Vidal, Opt. Commun. 200, 1 (2001); A. Benabbas, V. Halté, and J. Y. Bigot, Opt. Express 13, 8730 (2005).

${ }^{35}$ D. R. Smith, D. C. Vier, T. Koschny, and C. M. Soukoulis, Phys. Rev. E 71, 036617 (2005).

${ }^{36}$ Z. Huang, J. Xue, Y. Hou, J. Chu, and D. H. Zhang, Phys. Rev. B 74, 193105 (2006).

${ }^{37}$ C. García-Meca, R. Ortuño, F. J. Rodríguez-Fortuño, J. Martí, and A. Martínez, Opt. Express (to be published). 



\section{Chapter 4}

\section{Enlarging the negative-index bandwidth}

\section{by hybridized plasmon resonances}

Since Veselago predicted the existence of unconventional phenomena for NIMs, much effort has been devoted to the practical realization of such media. Because of the impossibility of natural media to show a simultaneously negative electric and magnetic response in the same spectral range, NIMs need to be artificially synthesized from subwavelength building blocks in order to show an effective material behavior, with a negative index of refraction. However, the negativeindex region is achieved over a limited frequency range as most of the designs rely on resonant effects, such as the case of the fishnet metamaterial. In order to circumvent this limitation, in this chapter a fishnet metamaterial is designed to show a negative effective index within a large frequency bandwidth based on the hybridization of the internal SPP modes.

\subsection{Introduction}

In the previous chapter it has been shown that for normal propagation, the fishnet behaves as a resonant magnetic-moment element, created by antiparallel resonant currents when a SPP is excited at the inner fishnet's metallic interfaces, referred to as an internal SPP (I-SPP). If this resonant magnetic-moment, that yields a negative effective permeability, occurs in a frequency region where the effective permittivity is also negative, a negative refractive index is achieved under normal propagation. However this behavior is limited to a reduced frequency region, thus hindering the practical implementation of such metamaterials in broadband applications. 
In this chapter, the concept of I-SPP resonances is exploited in a simple and practical approach to enlarge the negative-index bandwidth of the fishnet metamaterial. The I-SPP resonant frequency can be tuned by adjusting, among other parameters, the lattice and dielectric thickness slab comprising the fishnet. Hence, stacking several fishnet layers with different dielectric thicknesses will excite I-SPP resonances at slightly different frequencies in each fishnet layer. As long as the selected resonances are close enough, an electromagnetic coupling will appear between neighboring fishnet layers yielding a plasmon hybridization and new resonances to appear [PRO-03], [NOR-04], [WAN-06], [LI-06], [LIU-07], [LIU08b]. Precisely, this plasmon hybridization effect enables us to enlarge the bandwidth at which a negative effective refractive index is achieved. Additionally, because of the coupling effects, the evolution of the optical properties of the NIM is reported as a function of the stacked fishnet layers until the convergence of the optical parameters seems to be reached.

\subsection{Numerical results}

The stacked fishnet metamaterial considered in the calculations is depicted in Fig. 1 and is constructed from alternating metallic fishnet layers and dielectric spacers. Only the silver layer is drilled by the square lattice of rectangular holes filled with air. As the unit cell comprises dielectric spacers with two different thicknesses alternately stacked, a total of $4 N+1$ alternating metallic and dielectric layers are needed to achieve $N$ functional metamaterial layers.

For the metallic and dielectric layers silver and a permittivity of $\varepsilon=2.25$, such as the one of silica or SU8 polymer, were considered in the numerical calculations. The calculated spectral response, under normal plane-wave incidence, is shown in Fig. 2 with up to $N=4$ functional metamaterial layers in order to investigate the resonant behavior and optical properties with the number of stacked layers.

When the structure is comprised of only one functional metamaterial layer, four resonances appear in the spectral response. The origin of the labeled $1_{a}$ and $1_{b}$ resonances corresponds to the coupling of the impinging light to I-SPP modes when the parallel momentum provided by the periodicity equals that of the ISPP, which also depends on the dielectric thickness. Since two dielectric thicknesses are employed, each resonance is ascribed to an I-SPP excitation in each dielectric spacer. The electromagnetic field distributions at those resonances illustrated in Figs. 3 clearly show how depending on the resonant frequency, the fields are concentrated in each one of the dielectric spacers, as expected for the corresponding I-SPP. Moreover, the close proximity of the neighbor dielectric 
spacers results in a coupling effect between the two I-SPP resonances, yielding the electromagnetic fields to oscillate antiphase and in-phase with respect to one another, a typical signature of plasmon hybridization [PRO-03], [NOR-04], [WAN-06], [LI-06], [LIU-07], [LIU-08b]. Hence, the effect of stacking successive functional metamaterial layers is to introduce additional hybridized modes, as well as to spread the range of resonant frequencies, in accordance with Fig. 2.

The third resonance, around $250 \mathrm{THz}$ for the one functional layer case, corresponds to the localized hole resonance and redshifts when increasing the number of functional layers. Finally, the fourth resonance that appears around $290 \mathrm{THz}$ is ascribed to a higher-order I-SPP mode. Thus, all the resonances that appear, when stacking functional layers, above the localized hole resonance will correspond to hybridizations of higher-order I-SPP resonances, analogously to the lower-order I-SPP modes.

Despite the lattice exceeds half the wavelength, as long as the wavevector projection on the in-plane directions is small compared with the in-plane reciprocal lattice vector of the fishnet metamaterial, the propagation of light traveling along the vertical direction or within some angular range is dominated by the subwavelength vertical period and not by the in-plane period [VAL-08]. Thus, there is only a single propagating mode in the negative-index region, allowing the effective metamaterial parameters to be retrieved [CHE-04] from the numerical complex coefficients of transmission and reflection data. The real components of the permeability $\mu$, refractive index $n$ and the figure of merit (FOM) are depicted in Fig. 4.

Additionally to the published results, Fig. 14 presents the evolution of the effective parameters with the number of functional layers showing the imaginary part of the permittivity negative values. Obviously, the problem of retrieving the effective metamaterial parameters is generally not unique, and physical conditions have to be imposed. For instance, $\varepsilon$ and $\mu$ must not reveal discontinuous spectral jumps and, for a passive medium, $\operatorname{Im}(n)>0$ must hold. However, as electric and magnetic dipoles are not independent in metamaterials, this condition can be fulfilled while, e.g., $\operatorname{Im}(\varepsilon)<0$ close to the magnetic resonance [DOL-06]. However, the fact that imaginary part of the permittivity is negative (or in the general case, $\operatorname{Im}(\varepsilon)$ and $\operatorname{Im}(\mu)$ exhibit opposite sign) seems to contradict our physical criteria, and that it violates the causality principle. Nevertheless, the negative sign of $\operatorname{Im}(\varepsilon)$ is not in contradiction with any physical law. In particular, the transmission losses (or dissipated energy), which should be always positive, does not require that $\operatorname{Im}(\varepsilon)$ and $\operatorname{Im}(\mu)$ must be simultaneously positive [KOS-03], [MAR-03b]. Thus, the negativeness of $\operatorname{Im}(\varepsilon)$, produced by the resonance- 
antiresonance coupling, is only an artifact arising from the periodicity [KOS-05], [KOS-05b].
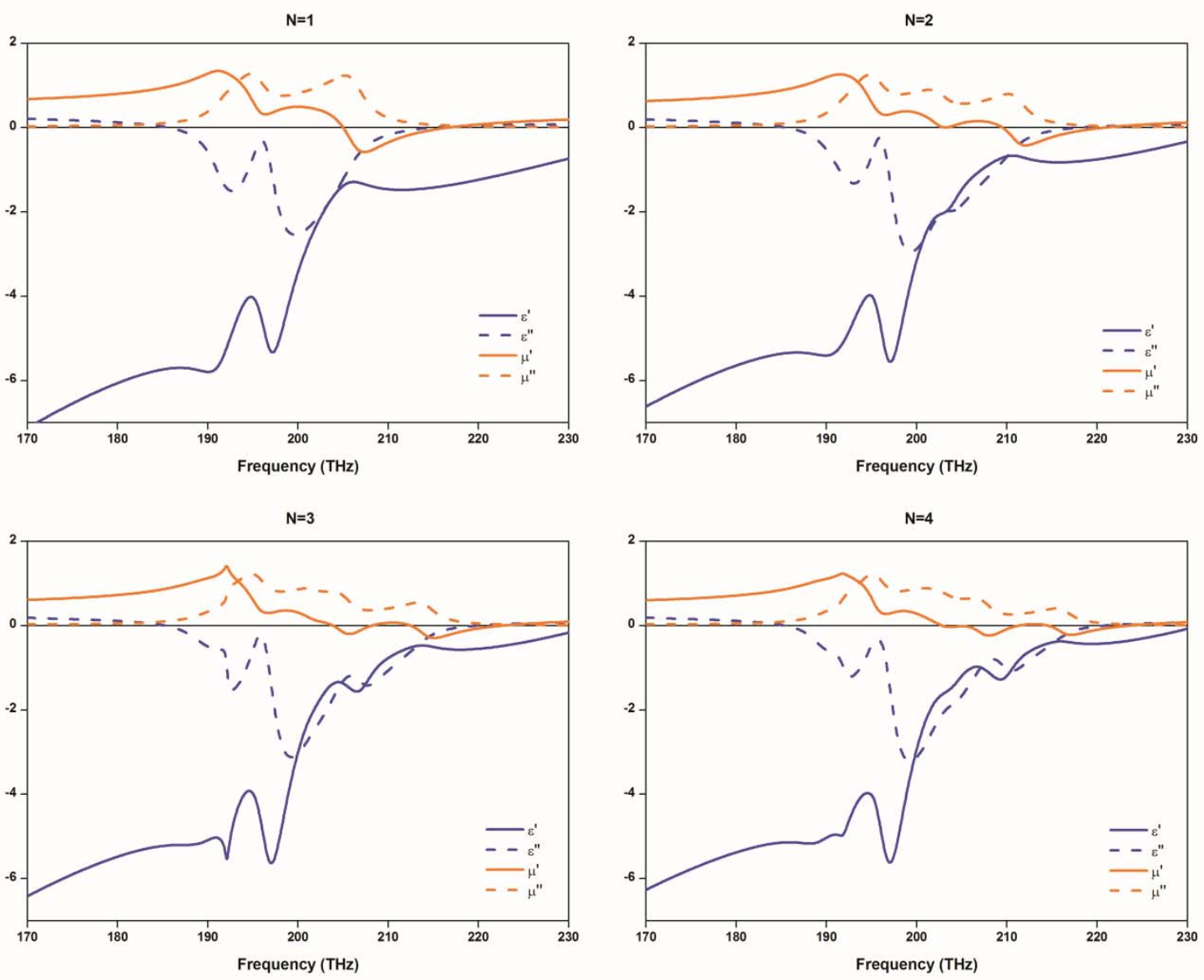

Figure 14. Evolution of the $\varepsilon$ and $\mu$ effective parameters with the number $N$ of functional layers.

All the magnetic resonances are obtained at those frequencies at which a hybridized I-SPP is excited, because of the formation of virtual current loops counteracting with the incoming magnetic field. However, $\mu$ does not become negative in all the resonances. The reason is that the resonances correlated with the excitation of fully symmetric plasmon modes can strongly counteract the external electromagnetic field and therefore lead to stronger negative magnetic responses, while the antiphase plasmon modes reduce the magnetic moment and so contribute little to the permeability [LI-06], [LIU-08b]. On the other hand negative values of $\varepsilon$ are achieved below the localized hole resonances [GAR-09]. Therefore, at the frequency regions where both the real components of the permittivity $\varepsilon$ and permeability $\mu$ reach negative values, the metamaterial can be referred to as double-negative material (DNG) achieving a negative refractive index $n$ with high FOM. However, because of the sign of $n$ is determined by the expression $\operatorname{Re}(\varepsilon)|\mu|+\operatorname{Re}(\mu)|\varepsilon|$ [DEP-04], $n$ can still reach negative values at the frequencies where $\mu$ does not become negative, albeit at the cost of dramatically low FOM. 
Additionally, the evolution of the optical parameters with the number of stacked functional layers seems to converge despite the coupling effects between functional layers. Because of these hybridization effects, which lead the negative permeability to spread as the functional layers increase, the DNG range widens. Consequently, the negative index bandwidth and the FOM broaden. Interestingly, a negative refractive index is achieved over a broad spectral range of about 70 $\mathrm{THz}$ (approximately from 150 up to $230 \mathrm{THz}$ ).

This broad DNG-NIM behavior will not be possible when the dielectric spacers have the same thickness. For this case although a negative index is achieved, but within a narrower bandwidth, the metamaterial cannot be considered DNG any longer as the permeability does not reach negative values. Thus, corroborating the importance of properly tune the dielectric thicknesses in a fishnet stack in order to achieve straightforwardly a broad negative-index bandwidth with negative permeability values, leading to a DNG-NIM behavior over a broad spectral range.

These results seem to be a contradiction because when different dielectric thicknesses are employed, the number of the resonances increases on expense of increasing the size of the elementary cell. Therefore, the volume per resonance increases and the spatial density of resonating magnetic moments at a particular frequency decreases. In such situation one may intuitively expect the effective permeability to be smaller in comparison to the single resonance case (i.e. only one dielectric thickness considered) as the volume per resonance decreases. However, one should carefully study each particular case separately. For example, in this case, the greater unit cell size will indeed reduce the density of resonances, but at the same time it reduces the coupling between equal-frequency resonances, which is favorable for the convergence of the parameters when stacking unit cells. In fact, if only one dielectric thickness is used the coupling is so strong that the convergence of the parameters is not achieved and no negative values of permeability are obtained. Hence, using a bigger unit cell reduces coupling, improves convergence and achieves negative values of permeability.

\subsection{Conclusions}

As a conclusion, an alternative and straightforward approach to achieve a NIM over a broad spectral bandwidth is presented. The proposed metamaterial design is based on the close relationship between I-SPPs and the artificial magnetic response in fishnet structures. The hybridization of the I-SPP resonances in the cascaded fishnet metamaterial leads to broaden the magnetic resonant response that, in combination with a negative permittivity, can cause a broad negative 
refractive index response. The evolution of the optical response of the NIM structure as a function of the number of functional layers is also studied in order to build up a thick NIM that could be employed to develop practical device applications. 


\title{
Enlarging the negative-index bandwidth of optical metamaterials by hybridized plasmon resonances
}

\author{
R. Ortuño,* C. García-Meca, F. J. Rodríguez-Fortuño, and A. Martínez \\ Nanophotonics Technology Center, Universidad Politécnica de Valencia, 46022 Valencia, Spain \\ ${ }^{*}$ Corresponding author: ruormo@ntc.upv.es
}

Received August 11, 2010; revised October 29, 2010; accepted November 4, 2010; posted November 8, 2010 (Doc. ID 133273); published December 14, 2010

\begin{abstract}
By exploiting the concept of internal surface plasmon polariton (I-SPP) resonances, which appear at nonsingle metallic film stacks, we have designed a metamaterial showing a negative effective index within a large frequency bandwidth. The designed structure consists of an arrangement of several fishnet layers. By properly adjusting the lattice and the thickness of the dielectric slab of the fishnet, an I-SPP mode can be excited at a certain frequency, giving rise to a negative permeability. Thus, when combining several fishnet layers, each one configured to excite an I-SPP at a slightly different frequency, the coupling among the fishnet layers will cause a plasmon hybridization effect that enables us to extend the negative-index bandwidth. (C) 2010 Optical Society of America

OCIS codes: $\quad 050.6624,160.3918,240.6680,350.3618$.
\end{abstract}

Since Veselago predicted the existence of unconventional phenomena for negative-index materials (NIMs) [1], much effort has been devoted to the practical realization of such media given their peculiar physics, which results in different capabilities for the manipulation of electromagnetic waves. Because of the impossibility of natural media to show a simultaneously negative electric and magnetic response, NIMs need to be artificially synthesized from subwavelength building blocks in order to show an effective material behavior, with overlapping negative permittivity and permeability frequency bands giving rise to a negative index of refraction.

While the first and most of the current NIMs working in the microwave regime are systems based on split-ring resonators in combination with a metallic wire medium [2], the fishnet metamaterial is the most common NIM design employed at optical frequencies [3] . For normal propagation, the fishnet behaves as a resonant magnetic-moment element, where the magnetic moment is created by antiparallel resonant currents, yielding a virtual current loop when a surface plasmon polariton (SPP) is excited at the inner fishnet's metallic interfaces, referred to as an internal SPP (I-SPP) [4]]. On the other hand, the electrical response arises from the interaction of the electric field with the holes and the external metallic surfaces [5], rather than just from the strip areas oriented along the electric field direction, reducing the equivalent surface plasma frequency [6]. Thus a negative-index region is achieved over a limited frequency range, as it relies on resonant effects.

In this Letter, we suggest a simple and practical approach to enlarge the bandwidth of the negative-index region by considering different thicknesses for the dielectric layers comprising the fishnet design, so that I-SPP resonances will be excited at slightly different frequencies in each fishnet layer [4]. The frequency of the different I-SPP resonances is critical to the design of the enlarged bandwidth. By varying the corresponding dielectric thickness, each I-SPP resonance can be easily tuned to take place at the desired frequencies [4]. Thus, stacking several fishnet layers enables us to enlarge the bandwidth at which a negative effective refractive index is achieved, as long as the selected resonances are close enough. In addition, stacking of metamaterials confronts the problem of vertical electromagnetic coupling of neighboring metamaterial layers. This coupling contributes to new resonances appearing owing to plasmon hybridization [7-12]. In this sense, we report the evolution of the optical properties of the NIM as a function of the number of stacked unit cells.

Figure 1 schematically shows a stacked fishnet structure that is constructed from alternating layers of silver fishnets and dielectric spacers. The sample geometrical parameters are given in the caption of Fig. 1. Two different thicknesses $\left(s_{1}\right.$ and $\left.s_{2}\right)$ are considered for the dielectric spacer alternately stacked. Thus, in order to achieve $N$ functional metamaterial layers, a total of $4 N+$ 1 alternating silver and dielectric slabs are needed, as the unit cell comprises two pairs of silver fishnet and

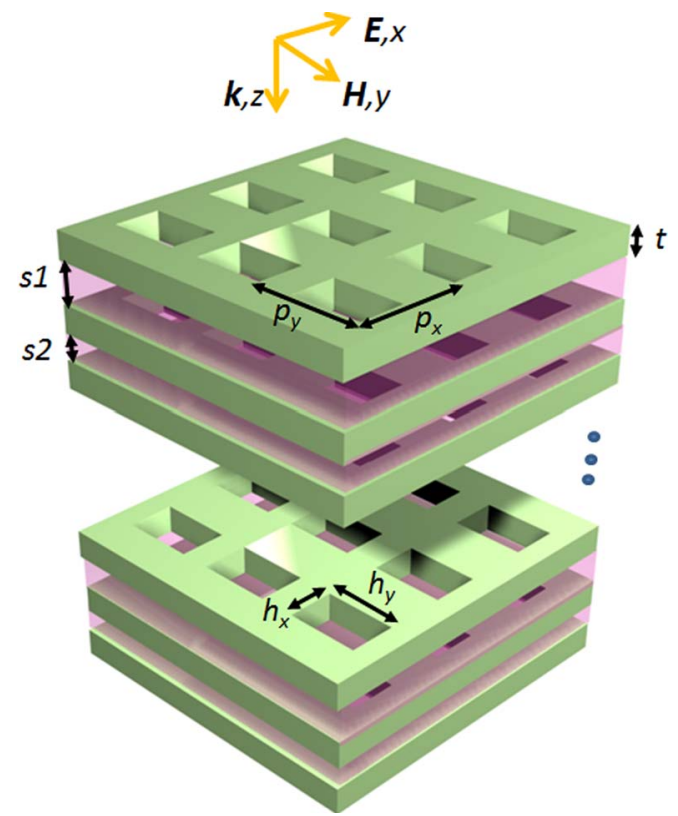

Fig. 1. (Color online) Scheme of the analyzed multilayer fishnet metamaterial and polarization configuration. The geometry parameters are: rectangular holes with dimensions $h_{x}=$ $280 \mathrm{~nm}$ and $h_{y}=453.5 \mathrm{~nm}$, lattice constant $p_{x}=p_{y}=650 \mathrm{~nm}$, silver thickness $t=100 \mathrm{~nm}$, and dielectric thicknesses of $s_{1}=$ $60 \mathrm{~nm}$ and $s_{2}=50 \mathrm{~nm}$. 
dielectric spacer layers alternately stacked. To investigate the resonant behavior and optical properties with the number of stacked layers, up to $N=4$ functional metamaterial layers have been studied. All numerical simulations were performed by employing CST software. The permittivity of silver is described by the Drude model with the plasma frequency $\omega_{p}=1.36 \times 10^{16} \mathrm{~s}^{-1}$ and the damping constant $\gamma=8.5 \times 10^{13} \mathrm{~s}^{-1}$. For the dielectric spacers, a dielectric permittivity of $\varepsilon=2.25$ (as in silica or in the SU8 polymer) was considered. Only the silver layer is drilled by the square lattice of rectangular holes filled with air, because better results are achieved with this configuration, rather than when the holes also go through the dielectric spacers. For all the calculations, we have restricted ourselves to normal plane-wave incidence, with the magnetic field pointing along the broad metallic strip areas of the fishnet.

The simulated reflection and transmission spectra for different functional metamaterials layers are presented in Figs. 2(a) and 2(b), respectively. As shown in Fig. 2(a), four resonances are observed for the one functional metamaterial layer. The origin of the two first resonances $\left(1_{a}\right.$ and $1_{b}$ ) corresponds to the coupling of the impinging light to I-SPP modes running along the inner metallicdielectric interfaces. This coupling occurs when the parallel momentum provided by the periodicity equals that of the I-SPP, which also depends on the dielectric thickness [4]. Since two dielectric thicknesses are employed, each resonance is ascribed to an I-SPP excitation in each dielectric spacer. The electromagnetic field distributions at resonances $1_{a}$ and $1_{b}$ are illustrated in Figs. 3(a) and $3(\mathrm{~b})$, respectively. It is clearly seen how, depending on the resonant frequency, the fields are concentrated in each one of the dielectric spacers, as expected for the corresponding I-SPP. Moreover, the close proximity of the neighbor dielectric spacers results in a coupling effect between the two I-SPP resonances, yielding the electromagnetic fields to oscillate antiphase and in-phase with respect to one another at resonances $1_{a}$ and $1_{b}$, respectively. This is a typical signature of plasmon hybridization [7-12]. Hence, the effect of stacking successive functional metamaterial layers is to introduce additional hybridized

(a)

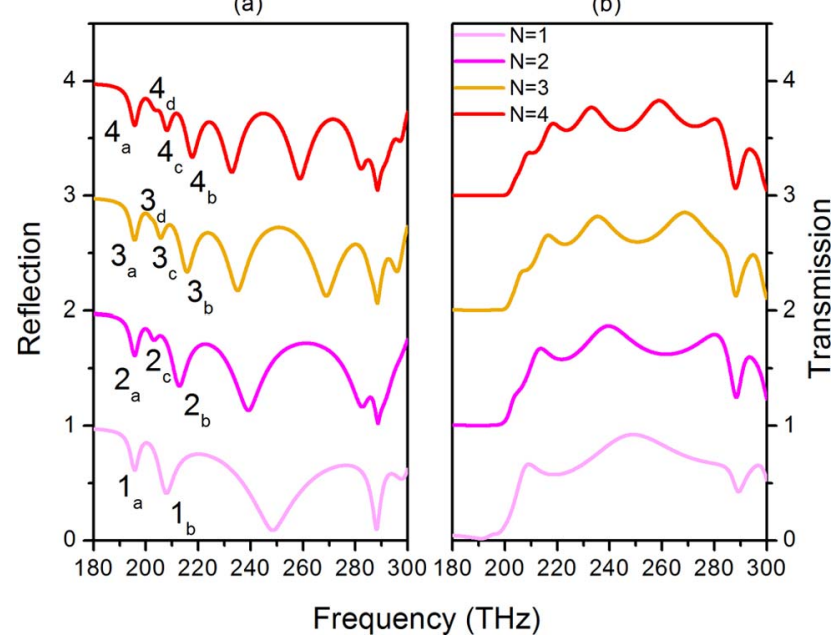

Fig. 2. (Color online) Simulated (a) reflection and (b) transmission spectra of one, two, three, and four functional metamaterial layers. Curves are shifted upward for clarity. (a)

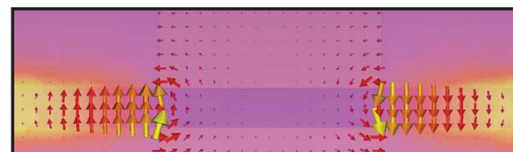

(b)

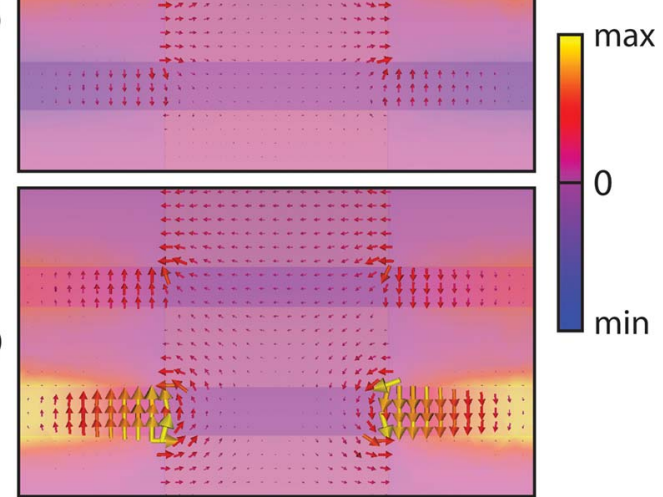

Fig. 3. (Color online) Simulated electromagnetic field distributions at resonance (a) $1_{a}$ and (b) $1_{b}$ in the plane $x=0$. The electric and magnetic fields are represented by arrows and color scale, respectively.

modes, as well as to spread the range of resonant frequencies, in accordance with Fig. 2 , as functional layers are stacked.

The third resonance, which appears around $250 \mathrm{THz}$ for the one functional layer case, corresponds to the localized hole resonance and shows a redshift when the number of functional layers increases. Finally, the fourth resonance that appears around $290 \mathrm{THz}$ is ascribed to a higher-order I-SPP mode. Thus, all the resonances that appear, when stacking functional layers, above the localized hole resonance will correspond to hybridizations of higher-order I-SPP resonances, analogously to the lowerorder I-SPP modes.

Although the subwavelength vertical periodicity justifies the description of the fishnet with effective metamaterial parameters [13], they can be strongly influenced by the number of stacked functional layers. Figure 4 shows the spectral behavior of the real components of the permeability $\mu^{\prime}$ and the refractive index $n^{\prime}$ and the figure of merit (FOM), which were retrieved from numerical complex coefficients of transmission and reflection data [14] for a different number of functional stacked layers. All the magnetic resonances of Fig. 4(a) are obtained at those frequencies at which a hybridized I-SPP is excited. These I-SPP excitations lead to the formation of virtual current loops, which counteract the incoming magnetic field and result in magnetic resonances. However, $\mu^{\prime}$ does not become negative in all the resonances. The reason is that the resonances correlated with the excitation of fully symmetric plasmon modes can strongly counteract the external electromagnetic field and therefore lead to stronger negative magnetic responses, while the antiphase plasmon modes reduce the magnetic moment and so contribute little to the permeability $[\underline{10}, 12]$. At the frequency regions where both the real components of the permittivity $\varepsilon^{\prime}$ and permeability $\mu^{\prime}$ reach negative values, the metamaterial can be referred to as double-negative material (DNG) and a negative refractive index $n^{\prime}$ with high FOM is achieved, as shown in Fig. 4(b). The DNG spectral ranges as a function of the number of functional layers are depicted with color bars in Fig. 4(a). It should 

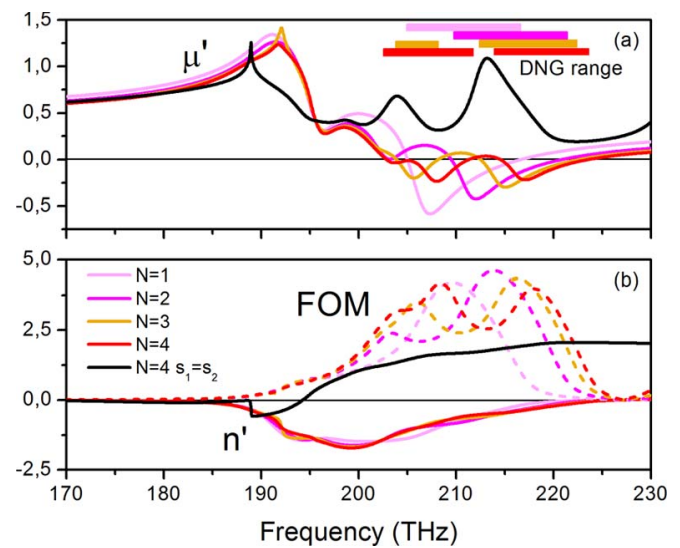

Fig. 4. (Color online) Retrieved real components effective material parameters of (a) permeability and (b) refractive index (solid curves) along with FOM (dashed curves) of one, two, three, and four functional metamaterial layers. The black curve represents the case when $s_{1}=s_{2}=50 \mathrm{~nm}$ and four functional layers are considered. The DNG spectral extensions as a function of the functional layers are shown in color bars in (a).

be stated that negative values of $\varepsilon^{\prime}$ are achieved below the localized hole resonances, as the electrical response arises from the interaction of the electric field with the holes and the external metallic surfaces [5]. Moreover, $n^{\prime}$ can still reach negative values at the frequencies where $\mu^{\prime}$ does not become negative. This is because the sign of $n^{\prime}$ is determined by the expression of $\varepsilon^{\prime}|\mu|+\mu^{\prime}|\varepsilon|$ [15]. Therefore, the occurrence of the negative refractive index at these frequencies is mainly attributed to the strong negative $\varepsilon^{\prime}$ and the positive $\mu^{\prime}$, albeit at the cost of dramatically low FOM. Additionally, the evolution of the optical parameters with the number of stacked functional layers seems to converge despite the coupling effects between functional layers. Because of these hybridization effects, which lead the negative permeability to spread as the functional layers increase, the DNG range widens. Consequently, the negative index bandwidth and the FOM broaden. Interestingly, a negative refractive index is achieved over a broad spectral range of about $70 \mathrm{THz}$ (approximately from 150 up to $230 \mathrm{THz}$ ). Finally, to check the advantages of the proposed structure, Fig. 4 shows the retrieved optical parameters when both dielectric spacers have the same thickness $\left(s_{1}=s_{2}=50 \mathrm{~nm}\right)$ and $N=4$ functional layers are considered. For this case a negative index is achieved, although the permeability does not reach negative values. However, the most interesting result is that a broader negative-index bandwidth with negative permeability values are achieved when different dielectric thicknesses are employed, leading to a DNG-NIM behavior over a broad spectral range. In doing so, the spatial density of resonating magnetic moments at a given frequency is reduced, which would intuitively re- duce the effective permeability compared to the case in which $s_{1}=s_{2}$. On the other hand, the greater distance between equal-frequency magnetic resonances reduces coupling and improves the convergence of the parameters when stacking unit cells.

In summary, we have presented an alternative approach to achieve a NIM that shows a negative refractive index over a broad spectral bandwidth. The proposed design is based on the close relationship between I-SPPs and the artificial magnetic response in fishnet structures. The hybridization of the I-SPP resonances in the cascaded fishnet metamaterial leads to broaden the magnetic resonant response that, in combination with a negative permittivity, can cause a broad negative refractive index response. The evolution of the optical response of the NIM structure with the number of functional layers is also studied in order to build a thick NIM that could be employed to develop device applications.

Financial support by the Spanish Ministry of Science and Innovation (MICINN) under contract nos. PET20070505, TEC2008-06871-C02-02, and CSD2008-00066 is gratefully acknowledged. The authors also acknowledge grant programs of Universidad Politécnica de Valencia, MICINN, and Generalitat Valenciana.

\section{References}

1. V. G. Veselago, Sov. Phys. Usp. 10, 509 (1968).

2. D. R. Smith, W. J. Padilla, D. C. Vier, S. C. Nemat-Nasser, and S. Schultz, Phys. Rev. Lett. 84, 4184 (2000).

3. S. Zhang, W. Fan, N. C. Panoiu, K. J. Malloy, R. M. Osgood, and S. R. J. Brueck, Phys. Rev. Lett. 95, 137404 (2005).

4. R. Ortuño, C. García-Meca, F. J. Rodríquez-Fortuño, J. Martí, and A. Martínez, Phys. Rev. B 79, 075425 (2009).

5. C. García-Meca, R. Ortuño, F. J. Rodriguez-Fortuño, J. Martí, and A. Martínez, Opt. Express 17, 6026 (2009).

6. J. B. Pendry, L. Martín-Moreno, and F. J. García-Vidal, Science 305, 847 (2004).

7. E. Prodan, C. Radloff, N. J. Halas, and P. Nordlander, Science 302, 419 (2003).

8. P. Nordlander, C. Oubre, E. Prodan, K. Li, and M. I. Stockman, Nano Lett. 4, 899 (2004).

9. H. Wang, D. W. Brandi, F. Le, P. Nordlander, and N. J. Halas, Nano Lett. 6, 827 (2006).

10. T. Li, H. Liu, F. M. Wang, Z. G. Dong, S. N. Zhu, and X. Zhang, Opt. Express 14, 11155 (2006).

11. N. Liu, H. Go, L. Fu, S. Kaiser, H. Schweizer, and H. Giessen, Adv. Mater. 19, 3628 (2007).

12. N. Liu, L. Fu, S. Kaiser, H. Schweizer, and H. Giessen, Adv. Mater. 20, 3589 (2008).

13. J. Valentine, S. Zhang, T. Zentgraf, E. Urin-Avila, D. A. Genov, G. Bartal, and X. Zhang, Nature 455, 376 (2008).

14. X. Chen, T. M. Grzegorczyk, B. I. Wu, J. Pacheco, and J. A. Kong, Phys. Rev. E 70, 016608 (2004).

15. R. A. Depine and A. Lakhtakia, Microw. Opt. Technol. Lett. 41, 315 (2004). 



\section{Chapter 5}

\section{EOT from dielectric-guided modes}

Besides the EOT peaks ascribed to SPPs excitation in subwavelength metallic aperture arrays, multiple transmission peaks also appear in the case of nanostructured metallic films embedded in dielectric claddings. A thoroughly numerical and theoretical study of such optical transmission resonances is carried out in this chapter, linking these transmission peaks with the excitation of FabryPerot modes sustained at the claddings, and coupled through the metal, as long as a periodic pattern is milled in the metal film. In addition, this structure is proposed to be used as an ultracompact all-optical switch by surrounding the metal film with Kerr nonlinear dielectric layers.

\subsection{Introduction}

Transmission of light through reflecting screens is typically associated with the excitation of some kind of resonance. For instance, the excitation of SPPs using prism couplers can provoke high transmission through opaque films [DRA-85] or even EOT from subwavelength apertures arrays [EBB-98]. Additionally, localized resonances observed in isolated holes and aperiodic arrays [KOE-04], or FabryPerot (FP) resonances due to the fact that propagating modes inside slits set up standing waves between the two ends of the slit [TAK-01] have been shown to play an important role in achieving EOT. Also, complete tunnelling of light through opaque flat slabs where only evanescent waves are allowed can be possible, as long as such a slab is surrounded by two identical slabs with high permittivity [ZHO-05].

This chapter is devoted to study metallic films milled with an aperture array that allows the coupling of the incident light to both the TE and TM modes supported by the dielectric claddings. The developed theoretical model 


\section{INTRODUCTION}

predicts the multiple peaks to come from FP resonances, evanescently coupled through the metal film as long as the metal periodicity is considered in the wavevector component tangential to the metal surface. In addition to these FP resonances, transmission peaks induced by SPP resonances due to the structure periodicity are also present in the spectrum.

Numerical simulations were performed to check the validity of the theoretical model, showing the effects of the dielectric claddings thickness and the periodic lattice on the light transmission through a subwavelength metallic hole array. The validity of the model is also supported by the fact that these transmission resonances are not related to either tunnelling transmission, since for flat metal films no enhanced transmission is observed or FP inside the holes as no propagating modes are allowed inside the metal film.

Fundamentally, the FP mode of the dielectric slab without metal film, also possesses multiple peaks. However, one advantage the structure considered here presents is that its transmission features can be used to implement optical filters more compact than those obtained with dielectric multilayers. But the possible applications are not only restricted to optical filters. For instance, the multiple EOT phenomenon can be employed for ultrafast all-optical switching [DAN-09]. Such functionality can be achieved, for instance, if the metal layer is surrounded by dielectric layers with high Kerr nonlinear coefficients, as proposed here.

\subsection{Numerical results}

The considered structure, shown in Fig. 1(a), consists of a gold metal film milled with a square array of subwavelength holes surrounded at both sides by SU8 dielectric claddings. The simulated transmission spectrum corresponding to $4 \mu \mathrm{m}$ thick SU8 under normal incident radiation is depicted in Fig. 1(b) (solid line). Surprisingly, multiple peaks emerge in the spectra showing EOT. However, these multiple peaks are not expected to appear only from SPP theory. The absence resonances in the transmission spectrum through an unpatterned gold film (dashed line in Fig. 1(b)) reveals that the unexpected multiple resonances are neither due to tunnelling of light by evanescent waves in the gold film nor to FP modes in the SU8 slabs coupled through opaque flat metal slabs. In addition, the transmission level is very low as expected for a flat optically thick metal film. Thus, only when the structure is periodically patterned the multiple transmission peaks are observed, showing the influence of the lattice in the transmission through subwavelength metallic hole arrays.

In order to understand the physical origin of the unexpected peaks the conditions required for light transmission from FP resonances are derived 
theoretically when a monochromatic TM polarized plane wave impinges in the structure depicted in Fig. 2. For simplicity, the transmission coefficient as a function of the frequency and the transversal wavevector $\left(k_{x}\right)$ is calculated in a flat metal slab with no holes. Only then, the periodicity is considered in the transversal plane by forcing $k_{x}$ to have the values given by the reciprocal lattice vectors associated to the periodicity in the metallic hole array. Although this procedure has to be considered as an approximation because the scattering effects caused by the holes are ignored, the predictions of the model are very accurate. In that sense, in analogy with the well accepted model to predict the SPP dispersion relation for single layer hole arrays [GEN-07], [RAE-86], [GHA-98], this model provides a simple means to predict analytically an approximate resonant frequency for the EOT peaks coming from the excitation of FP modes without requiring the use of more complex methods that consider the scattering of the holes.

In the expressions defining the electromagnetic fields sustained in the structure (Eqs. (1)-(4)) no SPP excitation is considered as long as the cladding wavevector normal component remains a real value. On the other hand, in the considered frequency range the fields are evanescent in the air implying that the supported electromagnetic fields correspond to guided modes only in the claddings giving rise to FP modes, as shown in the insets of Fig. 3 This is the reason why there is no possibility for incoming light to couple to the FP modes in flat metal slabs, but possible when the metal is drilled with a periodical pattern due to the additional momentum given by the periodicity. It has to be stressed that it is the periodicity of the hole array what enables the coupling from external light to guided modes and, as a result, the multiple EOT peaks. If the holes were randomly distributed, external light would not couple to these modes [MAR-05], and only resonances due to the features of isolated holes would be observed [RUA-06].

The great accuracy between the numerical and theoretical results to predict the unexpected EOT resonances can be inferred from Fig.3, where transmission coefficient $T\left(f, k_{x}\right)$ is plotted, just considering the values in $k_{x}$ associated to the periodicity in the metallic hole array, for which light coupling can be possible. However, the predicted resonant frequencies are slightly larger than those obtained in simulations because the scattering effects produced by the presence of the holes are not taken into account in our model [GEN-07].

The variations in the resonant frequencies of the transmission peaks as a function of the cladding thickness for both numerical and theoretical calculations are depicted in Fig. 4, increasing the number of resonances with the cladding thickness. 
Based on the results accuracy, it can be definitely stated that the multiple transmission peaks observed in the spectra are related to the cladding supported FP modes which are evanescently coupled through the periodically patterned metal slab. In addition, it should be emphasized that for flat metal films no peaks are observed either in the transmission coefficient $T$ of the proposed model or in the simulated spectra. In that way, the holes play a fundamental role in the transmission and in facilitating the FP coupling by means of evanescent coupling through the holes.

\subsection{Ultrafast all-optical switching application}

The multiple EOT phenomenon can be employed for ultrafast all-optical switching if the metal layer is surrounded by dielectric layers with high Kerr nonlinear coefficients. In such configuration (see Fig. 5 inset), the high optical intensities inside the dielectric layers, as a consequence of the strong confinement of the field at the metal-dielectric interfaces, will provoke an instantaneous variation of the refractive index of the dielectric medium due to the Kerr effect.

If we assume that there are two different transmission windows (centred at $\lambda_{1}$ and $\lambda_{2}$ respectively) arising from the multiple EOT phenomenon, we can use a high-power pulsed pump signal (tuned at $\lambda_{1}$ ) to switch a probe signal (tuned at $\lambda_{2}$ ), as depicted in Fig. 5. When the pump signal is on, the index of the dielectric layers is instantaneously increased owing to the Kerr effect, which results in a red-shift of the EOT peaks. This produces an immediate effective switching of the probe controlled by the pump signal. Compared to other all-optical switching approaches, this structure has the important advantage of being extremely compact in the longitudinal direction, being its size the total dielectric-metaldielectric thickness.

\subsection{Conclusions}

Multiple EOT through nanostructured embedded metallic films, a phenomenon independent of conventional tunnelling transmission, is demonstrated by theoretical analysis and numerical simulations. These resonances, which appear only in the case of periodically patterned metal films because light coupling is allowed, are ascribed to FP modes in the claddings evanescently coupled through the drilled metallic film. Consequently, these results are of great significance as they relate the important role of the periodicity not only to surface modes, like SPPs, but also to propagating modes in the dielectric claddings in the onset of EOT resonances, and also in the design and operation of highly compact optical devices that could become important building blocks in future nano-optical systems, such as ultrafast optical switching as proposed here. 


\title{
Multiple extraordinary optical transmission peaks from evanescent coupling in perforated metal plates surrounded by dielectrics
}

\author{
R. Ortuño,* C. García-Meca, F. J. Rodríguez-Fortuño, J. Martí, and A. Martínez \\ Nanophotonics Technology Center, Universidad Politécnica de Valencia, Camino de Vera s/n, 46022 Valencia, Spain \\ *ruormo@ntc.upv.es
}

\begin{abstract}
We study numerically and theoretically the optical transmission of nanostructured gold films embedded in dielectric claddings. We show how multiple transmission peaks appear as the claddings thickness increases. These transmission peaks come not only from surface plasmon polariton excitations but also from the excitation of Fabry-Perot modes sustained at the claddings, coupled through the metal, as long as a periodic pattern is milled in the metal film. We propose that this structure could be used as an ultracompact all-optical switch by surrounding the metal film with Kerr nonlinear dielectric layers.
\end{abstract}

(C2010 Optical Society of America

OCIS codes: (050.2230) Fabry-Perot; (050.6624) Subwavelength structures; (240.5420) Polaritons; (240.6680) Surface plasmons.

\section{References and links}

1. R. Dragila, B. Luther-Davies, and S. Vukovic, "High transparency of classically opaque metallic films," Phys. Rev. Lett. 55(10), 1117-1120 (1985).

2. T. W. Ebbesen, H. J. Lezec, H. F. Ghaemi, T. Thio, and P. A. Wolf, "Extraordinary optical transmission through sub-wavelength hole arrays," Nature 391(6668), 667-669 (1998).

3. K. J. Koerkamp, S. Enoch, F. B. Segerink, N. F. van Hulst, and L. Kuipers, "Strong influence of hole shape on extraordinary transmission through periodic arrays of subwavelength holes," Phys. Rev. Lett. 92(18), 183901 (2004).

4. Y. Takakura, "Optical resonance in a narrow slit in a thick metallic screen," Phys. Rev. Lett. 86(24), 5601-5603 (2001).

5. L. Zhou, W. Wen, C. T. Chan, and P. Sheng, "Electromagnetic-wave tunnelling through negative-permittivity media with high magnetic fields," Phys. Rev. Lett. 94(24), 243905 (2005).

6. V. Lomakin, and E. Michielssen, "Enhanced transmission through metallic plates perforated by arrays of subwavelength holes and sandwiched between dielectric slabs," Phys. Rev. B 71(23), 235117 (2005).

7. A. D. Rakic, A. B. Djurisic, J. M. Elazar, and M. L. Majewski, "Optical properties of metallic films for verticalcavity optoelectronic devices," Appl. Opt. 37(22), 5271-5283 (1998).

8. C. Genet, and T. W. Ebbesen, "Light in tiny holes," Nature 445(7123), 39-46 (2007).

9. H. Raether, Surface Plasmons on Smooth and Rough Surfaces and on Gratings (Springer, 1988).

10. H. F. Ghaemi, T. Thio, D. E. Grupp, T. W. Ebbesen, and H. J. Lezec, "Surface plasmons enhance optical transmission through subwavlength holes," Phys. Rev. B 58(11), 6779-6782 (1998).

11. A. Martínez, and J. Martí, "Negative refraction in two-dimensional photonic crystals: role of the lattice orientation and the interface termination," Phys. Rev. B 71(23), 235115 (2005).

12. Z. Ruan, and M. Qiu, "Enhanced transmission through periodic arrays of subwavelength holes: the role of localized waveguide resonances," Phys. Rev. Lett. 96(23), 233901 (2006).

13. E. N. Economou, "Surface plasmons in thin films," Phys. Rev. 182(2), 539-554 (1969).

14. B. Esembeson, M. L. Scimeca, T. Michinobu, F. Diederich, and I. Biaggio, "A high optical quality supramolecular assembly for third- order integrated nonlinear optics," Adv. Mater. 20(23), 4584-4587 (2008).

15. R. Spano, N. Daldosso, M. Cazzanelli, L. Ferraioli, L. Tartara, J. Yu, V. Degiorgio, E. Giordana, J. M. Fedeli, and L. Pavesi, "Bound electronic and free carrier nonlinearities in Silicon nanocrystals at 1550nm," Opt. Express 17(5), 3941-3950 (2009).

16. K. M. Dani, Z. Ku, P. C. Upadhya, R. P. Prasankumar, S. R. J. Brueck, and A. J. Taylor, "Subpicosecond optical switching with a negative index metamaterial," Nano Lett. 9(10), 3565-3569 (2009).

\section{Introduction}

Transmission of light through reflecting screens has always been fascinating. Such a phenomenon is typically associated with the excitation of some kind of resonances. In that

\#122996 - \$15.00 USD Received 19 Jan 2010; revised 26 Feb 2010; accepted 23 Mar 2010; published 31 Mar 2010

(C) 2010 OSA

12 April 2010 / Vol. 18, No. 8 / OPTICS EXPRESS 7893 
way, the excitation of surface plasmon polaritons (SPPs) using prism couplers can induce high transmission through opaque films [1]. High transmission from subwavelength apertures arrays has also been reported, where the extraordinary optical transmission (EOT) is widely related to SPPs excitation [2]. However, other types of excitations have been shown to play an important role in achieving EOT, such as localized resonances observed in isolated holes and aperiodic arrays [3], or Fabry-Perot (FP) resonances due to the fact that propagating modes inside slits set up standing waves between the two ends of the slit [4]. Also, complete tunnelling of light through opaque flat slabs where only evanescent waves are allowed can be possible, as long as such a slab is surrounded by two identical slabs with high permittivity [5].

Unlike other studies through perfect electrically conducting (PEC) plates perforated by doubly periodic array of subwavelength holes [6], in this letter we study the case of gold films milled with a square array of holes that allows the coupling of the incident light to both the TE and TM modes supported by the dielectric claddings. In that way, we present how the dielectric claddings thickness and the periodic lattice affect the light transmission through a subwavelength metallic hole array. Multiple transmission peaks appear when increasing the cladding thickness only if the metal film presents a periodic pattern. We present a model that predicts these multiple peaks to come from FP resonances, due to propagating (TE and TM) waves inside the claddings, evanescently coupled through the metal film as long as the metal periodicity is considered in the wavevector component tangential to the metal surface. These transmission resonances are not related to either tunnelling transmission, since for flat metal films no enhanced transmission is observed or FP inside the holes as no propagating modes are allowed inside the metal film. In addition to these FP resonances, transmission peaks induced by SPPs resonances due to the metal periodicity are also present in the spectrum. These transmission features can be used to implement optical filters more compact than those obtained with dielectric multilayers.

\section{Numerical and theoretical results}

The considered structure consists of a gold metal film milled with a square array of subwavelength holes with radius $a$ of $1 \mu \mathrm{m}$ surrounded at both sides by dielectric claddings with a variable thickness $d$. The lattice period, $P$, is $5.5 \mu \mathrm{m}$ and the gold film thickness, $d_{m}$, is $100 \mathrm{~nm}$. A schematic picture of the structure is shown in Fig. 1(a). In Fig. 1(b) we show the simulated transmission spectrum corresponding to a dielectric cladding thickness $d=4 \mu \mathrm{m}$ for normal incident radiation with its electric field $E$ pointing along the $x$ direction obtained with the licensed software CST Microwave Studio, a general purpose electromagnetic simulator based on the finite integration technique. The relative dielectric constant of gold is fitted by the Drude model with the bulk plasma frequency $\omega_{p}=1.36 \times 10^{16} \mathrm{rad} / \mathrm{s}$ and damping rate $\gamma=$ $10^{14} \mathrm{~Hz}$ [7]. Both cladding slabs are SU8 layers with $\varepsilon_{r}=2.25$. From the transmission spectrum of the periodic array (solid line) it can be seen how multiple peaks emerge showing extraordinary transmission. However, these multiple peaks are not expected to appear only from SPP theory. The transmission spectrum considering an unpatterned gold film (dashed line) reveals that these resonances are neither due to tunnelling of light by evanescent waves in the gold film nor to FP modes in the SU8 slabs coupled through opaque flat metal slabs. In addition, the transmission level is very low as expected for a flat optically thick metal film. Thus, only when the structure is periodically patterned the multiple transmission peaks are observed, showing the influence of the lattice in the transmission through subwavelength metallic hole arrays.

\#122996 - \$15.00 USD Received 19 Jan 2010; revised 26 Feb 2010; accepted 23 Mar 2010; published 31 Mar 2010 (C) 2010 OSA 12 April 2010 / Vol. 18, No. 8 / OPTICS EXPRESS 7894 
(a)

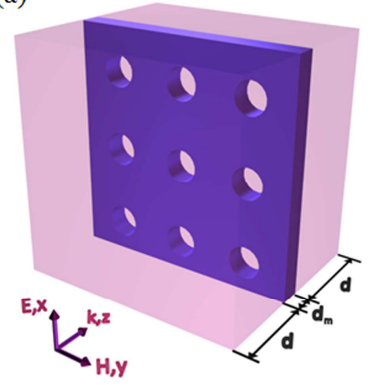

(b)

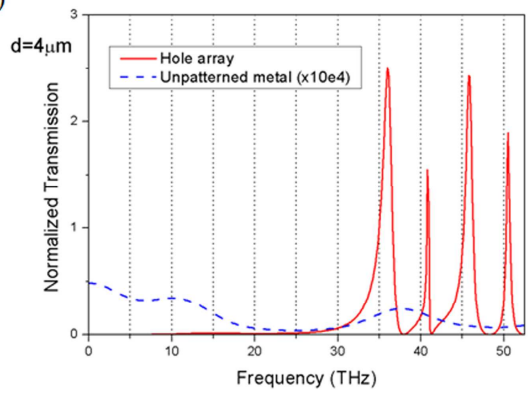

Fig. 1. (Color online) (a) Schematic view of the structure under study and the corresponding reference system used. (b) Transmission through a hole array (solid red line) and an unpatterned gold film (dashed blue line) with dielectric thickness $d=4 \mu \mathrm{m}$ obtained from numerical simulation.

On the theoretical side, in order to understand the physical origin of the unexpected peaks we derive the conditions required for light transmission from FP resonances through drilled metallic films sandwiched between two surrounding dielectric claddings. Figure 2 depicts a diagram of the FP scheme. A monochromatic transverse magnetic (TM) polarized plane wave is considered as the impinging light in the $x-z$ plane. For simplicity, we consider a flat metal slab with no holes to obtain the transmission coefficient $T$ as a function of the frequency and the transversal wavevector $\left(k_{x}\right)$. Only then, we consider the periodicity in the transversal plane by forcing $k_{x}$ to have the values given by the reciprocal lattice vectors associated to the periodicity in the metallic hole array. Although this procedure has to be considered as an approximation because the scattering effects caused by the holes are ignored, the predictions of the model are very accurate. In that sense, in analogy with the well accepted model to predict the SPP dispersion relation for single layer hole arrays [8-10], this model provides a simple means to predict analytically an approximate resonant frequency for the EOT peaks coming from the excitation of FP modes without requiring the use of more complex methods that consider the scattering of the holes.

Specifically, the fields in each layer considering propagating waves in the claddings slabs and evanescent in the metal film can be expressed as follows, where the common factor $\exp \left(j\left(k_{x} x-\varpi t\right)\right)$ is omitted in all the expressions:

$$
H_{y}=A_{l}^{+} e^{j k_{l} z}+A_{l}^{-} e^{-j k_{l} z}, \quad k_{l}=\left(k_{0}^{2}-k_{x}^{2}\right)^{1 / 2} .
$$

for $z \leq 0$,

$$
H_{y}=A_{i}^{+} e^{j k_{d} z}+A_{i}^{-} e^{-j k_{d} z}, \quad k_{d}=\left(k_{0}^{2} \varepsilon_{d}-k_{x}^{2}\right)^{1 / 2} .
$$

for the two $(i=1,2)$ claddings,

$$
H_{y}=B_{m}^{+} e^{k_{m} z}+B_{m}^{-} e^{-k_{m} z}, \quad k_{m}=\left(k_{x}^{2}-k_{0}^{2} \varepsilon_{m}\right)^{1 / 2} .
$$

for the metal film, and

$$
H_{y}=A_{t}^{+} e^{j k_{t} z}, \quad k_{t}=\left(k_{0}^{2}-k_{x}^{2}\right)^{1 / 2} .
$$

for $z \geq 2 d+d_{m}$. 
In the expressions (1)-(4) $k_{0}$ is the free-space wave number and $\varepsilon_{d}, \varepsilon_{m}$ are the relative permittivity of the claddings and the metal slab, respectively; $A_{n}^{+}\left(A_{n}^{-}\right)$represents the complex amplitude of the forward-propagating (backward-propagating) waves in the corresponding layer; $B_{m}^{+}\left(B_{m}^{-}\right)$denotes the forward-decaying (backward-decaying) evanescent wave in the metal slab. In particular, $A_{t}^{+}$corresponds to a transmission amplitude $t$. The square of its magnitude corresponds to the transmission $T$ coefficient, respectively.

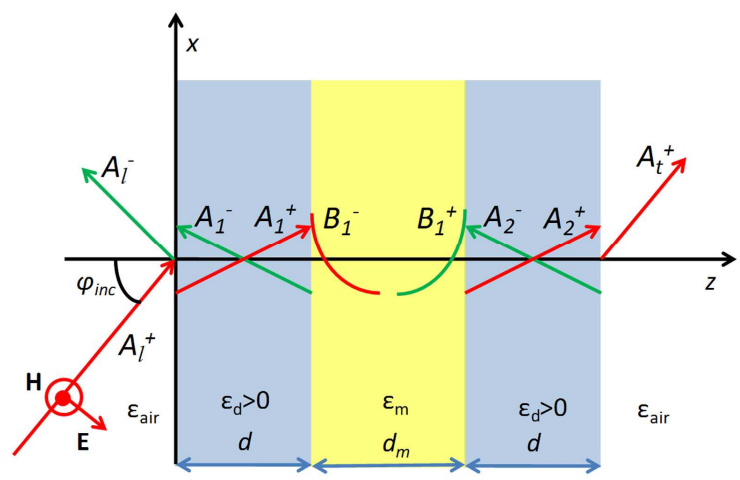

Fig. 2. (Color online) Schematic diagram of the FP configuration for a flat metal slab $\left(\varepsilon_{m}, d_{m}\right)$ surrounded with dielectric claddings $\left(\varepsilon_{d}, d\right)$.

It should be remarked that with this electromagnetic scheme no SPP excitation is considered as long as $k_{d}$ remains a real value. However, in the considered frequency range $k_{l}$ takes imaginary values. Consequently, the fields are evanescent in the air implying that the supported electromagnetic fields correspond to guided modes only in the dielectric cladding giving rise to FP modes. Thus, there is no possibility for incoming light to couple to the FP modes in flat metal slabs. However, the coupling is possible when the metal is drilled with a periodical pattern due to the additional momentum given by the periodicity. It has to be stressed that it is the periodicity of the hole array what enables the coupling from external light to guided modes and, as a result, the multiple EOT peaks. If the holes were randomly distributed, external light would not couple to these modes [11], and only resonances due to the features of isolated holes would be observed [12]. Figure 3 shows the calculated $T\left(f, k_{x}\right)$ coefficient for the same dielectric thickness as in Fig. 1(b), where $T$ was rescaled for a better representation. The insets show how the sustained modes are propagating in the dielectric and evanescent in the air (they lay between the vacuum and dielectric light lines). Now, the unexpected EOT peaks appeared in the transmission spectrum of Fig. 1(b) can be explained if we just consider the values in $k_{x}$ associated to the periodicity in the metallic hole array, for which light coupling can be possible. With this consideration, it is inferred from Fig. 3 that transmission is achieved at $37.77 \mathrm{THz}, 41.21 \mathrm{THz}, 48.16 \mathrm{THz}$ and $53.2 \mathrm{THz}$, in close similarity to the ones obtained in numerical simulation at $36.07 \mathrm{THz}, 40.88 \mathrm{THz}, 45.58 \mathrm{THz}$ and $50.33 \mathrm{THz}$. The predicted resonant frequencies are slightly larger than those obtained in simulations because the scattering effects produced by the presence of the holes are not taken into account in our model [8]. It should be remarked that although the $\mathrm{TM}_{0}$ mode is above the dielectric light line, it becomes a SPP mode when the dielectric thickness increases.

Figure 4(a) shows the simulated light transmission spectra, as a function of the cladding thickness $d$, through the subwavelength metallic hole array for a normal incoming plane wave. The variations of the peaks resonant frequencies show the spectra dependence on the cladding thickness and how more transmission peaks appear when increasing $d$. These multiple transmission peaks are not all expected to appear only from SPPs theory. On the other hand, Fig. 4(b) shows the variation of the theorized transmission coefficient $T$ with the cladding thickness $d$, after considering the periodicity of the drilled metallic hole array in the transversal wavevector component $k_{x}$. The flat line around $36 \mathrm{THz}$ is a numerical artifact 
which corresponds to the trivial solution of the dielectric light line corresponding to the case when all fields are zero [13]. The same scale factor is also used again to represent $T$.

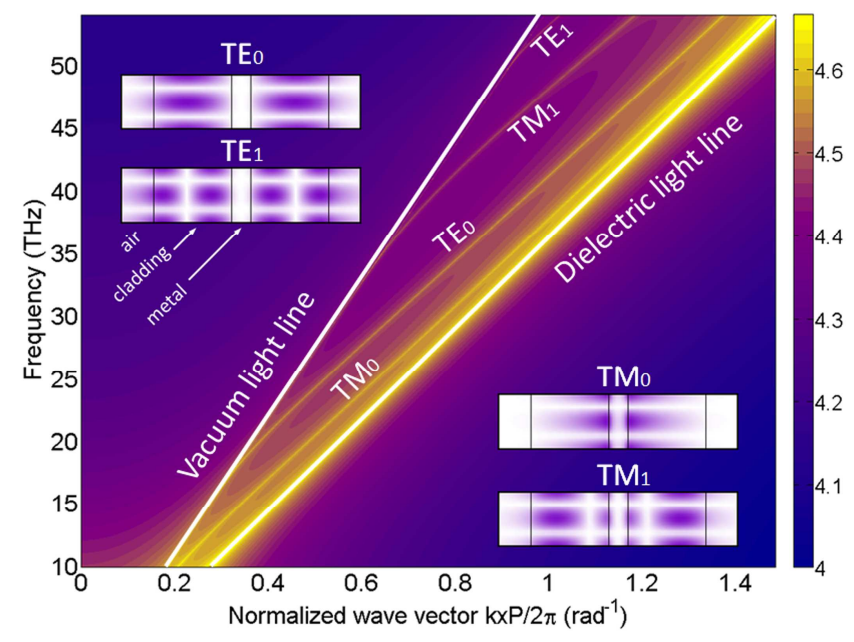

Fig. 3. (Color online) Representation of the theoretical transmission coefficient $T\left(f, k_{x}\right)$ for a dielectric thickness of $d=4 \mu \mathrm{m}$. Insets: electric and magnetic fields for TE and TM modes respectively in a structure scaled for better representation.

Comparing Figs. 4(a) and 4(b), leaving the scale factor aside, it can be definitely stated that the multiple transmission peaks observed in the spectra are related to the cladding supported FP modes which are evanescently coupled through the periodically patterned metal slab. In addition, it should be emphasized that for flat metal films no peaks are observed either in the $T$ coefficient of the proposed model or in the simulated spectra. In that way, the holes play a fundamental role in the transmission and in facilitating the FP coupling by means of evanescent coupling through the holes.

(a)

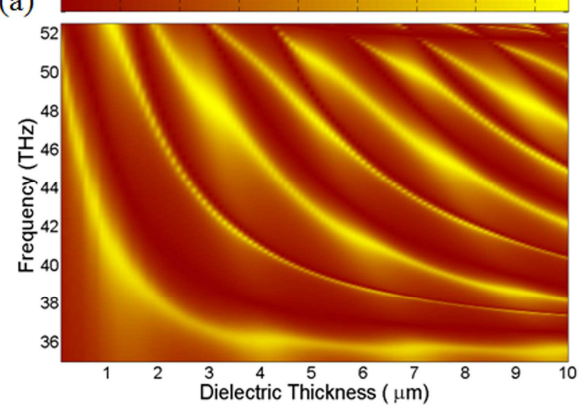

(b)

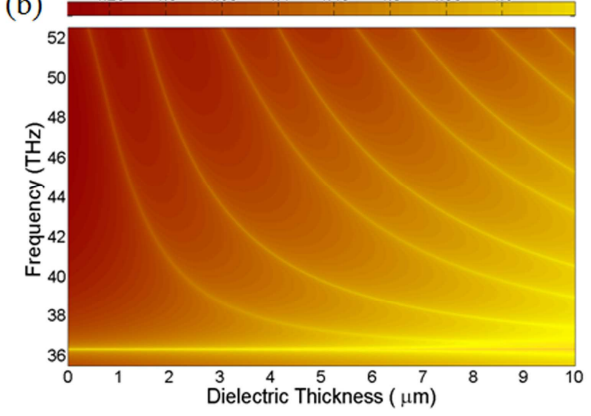

Fig. 4. (Color online) (a) Simulated transmission spectra vs. dielectric thickness $d$. (b) The same for the calculated transmission spectra $T$ after considering the $k_{x}$ values associated with the periodicity.

\section{Application to ultrafast all-optical switching}

The existence of multiple EOT peaks can be used in several applications. Here, we propose that multiple EOT phenomenon can be employed for ultrafast all-optical switching if the metal layer is surrounded by dielectric layers with high Kerr nonlinear coefficients (such as nonlinear polymers [14] or silicon nanocrystals in a silica matrix [15]). The Kerr effect is an instantaneous variation of the refractive index of a dielectric medium proportional to the 
optical intensity (square of the electrical field). In the structure under consideration (see Fig. 5 inset), high optical intensities inside the dielectric layers are expected even for moderate power levels as a consequence of the strong confinement of the field at the metal-dielectric interfaces. If we assume that there are two different transmission windows (centred at $\lambda_{1}$ and $\lambda_{2}$ respectively) arising from the multiple EOT phenomenon, we can use a high-power pulsed pump signal (tuned at $\lambda_{1}$ ) to switch a probe signal (tuned at $\lambda_{2}$ ), as depicted in Fig. 5. When the pump signal is on, the index of the dielectric layers is instantaneously increased owing to the Kerr effect, which results in a red-shift of the EOT peaks. This produces an immediate effective switching of the probe controlled by the pump signal. Compared to other all-optical switching approaches, this structure has the important advantage of being extremely compact in the longitudinal direction, being its size the total dielectric-metal-dielectric thickness. It has to be mentioned that a similar approach (a drilled metal-dielectric-metal structure) has been recently demonstrated to produce subpicosecond all-optical switching by making use of free carriers generated optically [16].

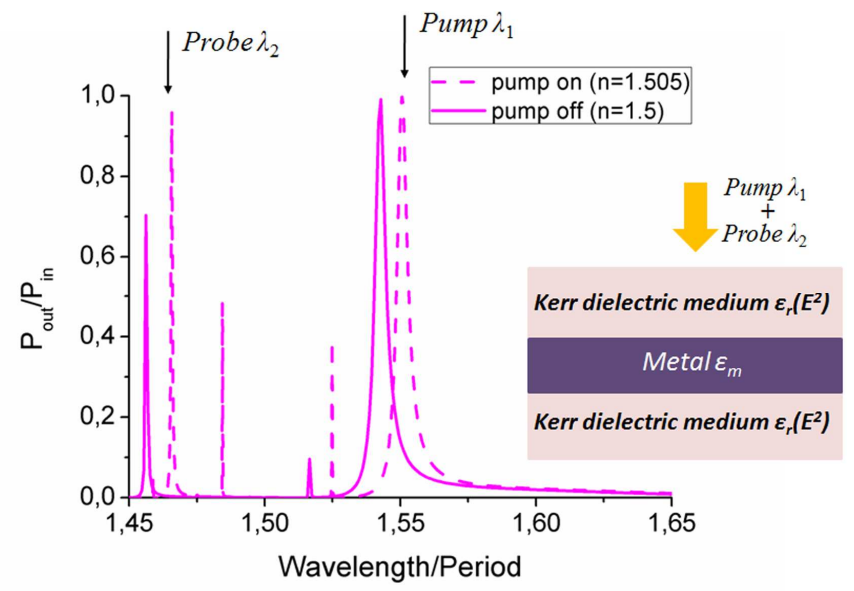

Fig. 5. (Color online) All-optical switch based on multiple EOT through a perforated metal plates surrounded by Kerr nonlinear dielectric media. Pump and probe optical signals are tuned at two different transmission windows $\left(\lambda_{1}\right.$ and $\left.\lambda_{2}\right)$. The Kerr effect is modelled by slightly increasing the dielectric refractive index $n$ : Pump on $n=1.505$, Pump off $n=1.5$. Inset: Scheme of the switching structure, which is extremely compact in the longitudinal direction.

\section{Conclusion}

In conclusion, through theoretical analysis and numerical simulations, we have demonstrated a mechanism for multiple extraordinary light transmission through a classically opaque material independent of conventional tunnelling transmission. The present transmission is characterized by FP modes in the claddings evanescently coupled through the drilled metallic film. Moreover, SPP excitations are also present in the transmission spectrum since the $\mathrm{TM}_{0}$ mode lies at the right of the dielectric light line for thicker dielectric claddings. Consequently, these results are of great significance as they relate the important role of the periodicity not only to surface modes, like SPPs, but also to propagating modes in the dielectric claddings in the onset of EOT resonances, and also in the design and operation of highly compact optical devices that could become important building blocks in future nano-optical systems.

\section{Acknowledgments}

Financial support by the Spanish MICINN was provided under contracts PET2007-0505, TEC2008-06871-C02-02, and CSD2008-00066. Consolider EMET is gratefully acknowledged. R. Ortuño, C. García-Meca, and F. J. Rodríguez-Fortuño also acknowledge grant programs FPI of UPV, FPU of MICINN, and FPI of GVA, respectively. 


\section{Chapter 6}

\section{Implementation of EOT based filters at}

\section{midinfrared range}

This chapter presents experimental measurement results of the EOT through nanostructured metallic films in the midinfrared region. Several samples are fabricated varying their defining physical parameters in order to investigate the influence of the surrounding claddings, hole shape, and periodicity on the resonance wavelength and the quality factor. The aim is to use the subwavelength structures as ultracompact optical filters whose spectral features can be easily tuned and scaled. The results show that the main parameters affecting the resonance wavelength are the lattice constant and dielectric cladding. On the other hand, the hole shape and size are found to cause transmission enhancement, a small resonance redshift and a lowering in the quality factor when the hole area is increased.

\subsection{Introduction}

The optical excitation of SPPs using attenuated total reflection [KRE-68], [OTT68] allowed SPP to be studied and their main properties assessed [RAE-86], [BOA-82]. However, the interest on SPP boosted when EOT excitation was reported [EBB-98]. Since then, subwavelength hole array structures periodically patterned on a metal film have attracted great attention owing to both its intriguing physical properties, in apparent contradiction with standard aperture theory [BET-44], and its technological potential in areas such as light manipulation at nanoscale, optical resolution below the diffraction limit, highly compact optical filters, gas detection and biosensing. The SPP unique properties, 


\section{INTRODUCTION}

which enable a wide range of practical applications, have lead to a surge of research and development activity related to SPP based structures and devices over the recent decades.

Although an open debate about the physical origin of EOT still exists. It is now widely accepted that EOT is related to the existence of surface waves supported by perforated metallic films and diffraction modes generated by periodic arrays of holes. In the optical regime, surface waves are SPPs; whereas in the microwave and terahertz frequencies plasmon-like surface waves are supported by interactions between holes even when metals behave as perfect conductors [PEN-04].

This chapter presents experimental measurements of EOT in the midinfrared range (MIR) of several fabricated subwavelength structures. The measured transmission spectrum shows how the grating momentum appears to define the main resonances of the spectrum. This confirms that EOT features are mainly due to the resonant excitation of SPPs by incident light. The simplicity with which the spectral features can be tuned and scaled has generated considerable interest and promoted subwavelength structures as a core element of modern optical devices. In this sense, the aim is to design ultracompact optical filters in the MIR region. Several samples with various hole sizes and shapes, surrounding claddings and lattice periodicity were fabricated in order to investigate their influence on the main characteristics defining a filter: i.e., the resonance wavelength $\lambda_{0}$; and the quality factor, defined as $Q=\lambda_{0} / \Delta \lambda$, where $\Delta \lambda$ is the full width at half maximum of the transmission resonance.

The reduce dimensions of the EOT filters and its selective spectral response makes them suitable candidates for ultra-compact filter applications. Specifically, they can be used for gas detection in the MIR range because of the fact that many hazardous gases present their spectral fingerprint in such spectral range, as Fig. 15(up) shows. In addition, their CMOS compatible fabrication and scalability results in mass production of large pixel-filter-arrays using the exact same process as a single filter at low cost feasibility, depicted in Fig. 15(bottom). 

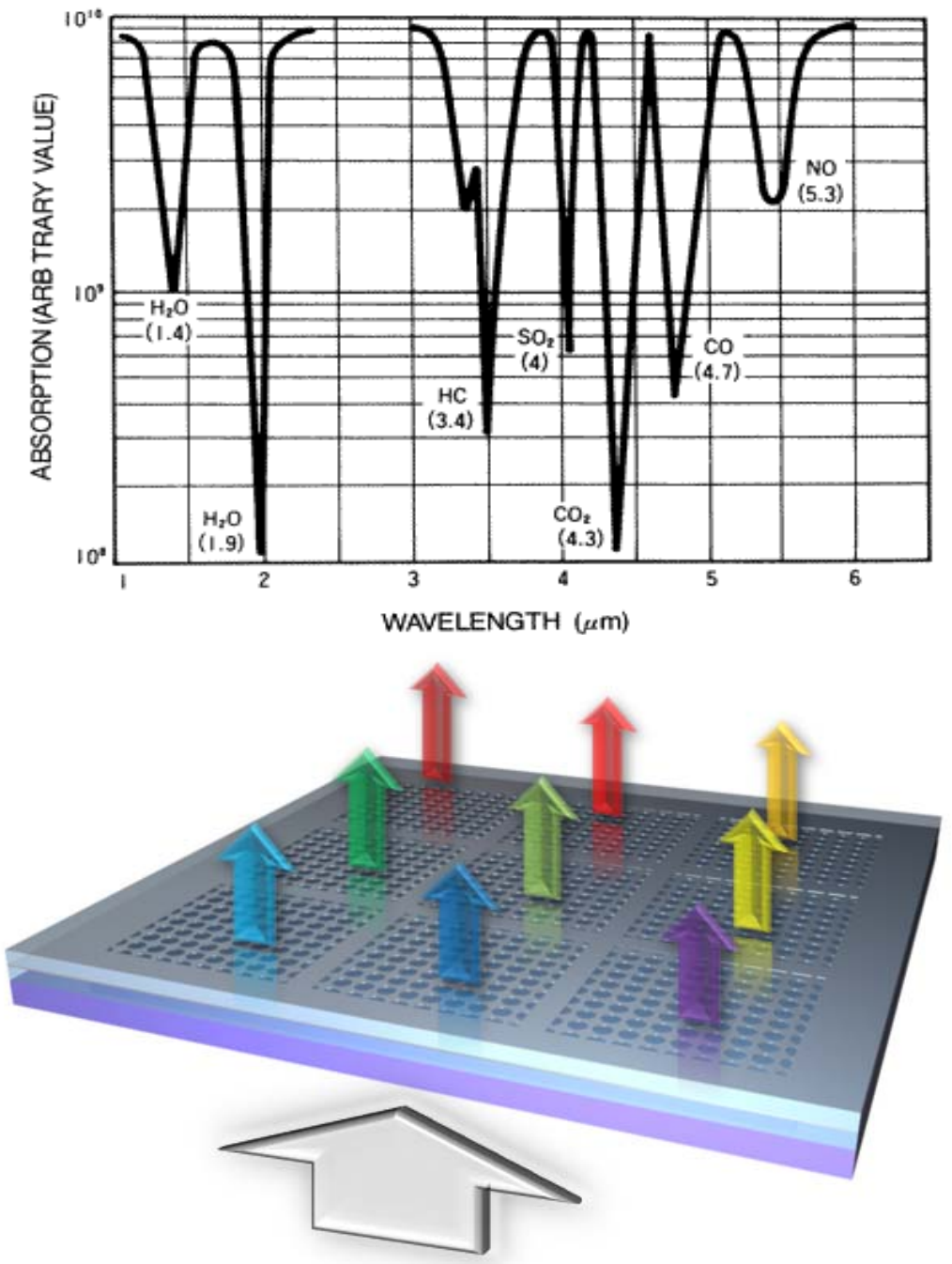

Figure 15. (Up) Spectral fingerprint of hazardous gases in the MIR range. (bottom) Artist vision of a filter array implemented with EOT structures.

\subsection{Fabrication and characterization processes}

The subwavelength hole arrays were made by vacuum evaporation of a thin gold film on a transparent silicon substrate wafer for characterization in the MIR region. Moreover, in order to characterize the cladding influence on the transmission spectrum, the nanostructured gold films were fabricated both directly over the Si wafer, surrounded by SU8 claddings on both sides and with only one SU8 cladding. The general prototype in which the metal film is sandwiched between claddings is illustrated in Fig. 16. 


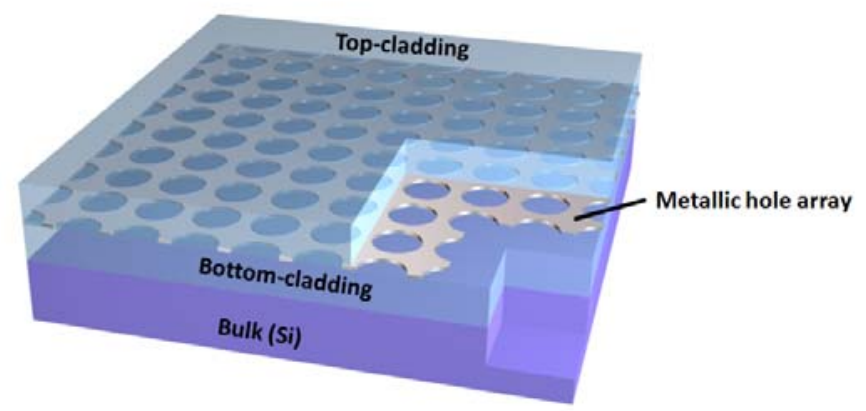

Figure 16. Schematic representation of a metallic hole array surrounded by top and bottom dielectric claddings over a Si wafer.

Among the experimental methods for structuring these thin films laterally electron-beam lithography was used as it has proven to be the most versatile. To illustrate the fabrication process, the steps followed to the fabrication of subwavelength metallic array structures embedded in SU8 claddings are resumed in the schematics of Fig. 17 (to follow a more specific representation see Fig. 2 of the publication). First, a clean silicon wafer is spin-coated with a $4.4 \mu \mathrm{m}$-thick SU8 polymer layer, a 5nm chromium layer, to provide adhesion for the gold film, and an electron-sensitive resist polymethyl methacrylate (PMMA) layer as shown in Fig. 2(a). Afterwards, the sample pattern is transferred to the electron resist via exposure to a focused electron beam and various periodic patterns can then be transferred simply by changing the dose rate. Subsequent chemical development removes the resist from all the regions exposed to the electron beam as schematically illustrated in Fig. 2(b). Gold metal is then deposited on the sample by a sputtering process as shown in Fig. 2(c). Following the deposition of the thin gold film, a chemical lift-off process removes the PMMA resist layer from the chromium surface. This left the periodic metallic structures deposited directly on the chromium-SU8 substrate through the openings in the resist mask. Finally, the spin-coating of a top cladding of another $4.4 \mu \mathrm{m}$-thick SU8 layer is performed. Since the symmetry of the structure is very important for the transmission properties, we carefully ensured that the cladding layers had the same refractive index. This was guaranteed by applying the same SU8 polymer for the top and bottom claddings; and using identical spinning and curing conditions. After the final SU8 polymer curing, the wafer shown in Fig. 2(d) was ready for optical characterization. The fabrication process for structures with only one SU8 cladding is identical to that previously described but without the last spin-coating deposition of the upper SU8 polymer cladding. Obviously, the bottom and upper SU8 polymer claddings deposition processes are not carried out for structures made directly on the silicon wafer. 


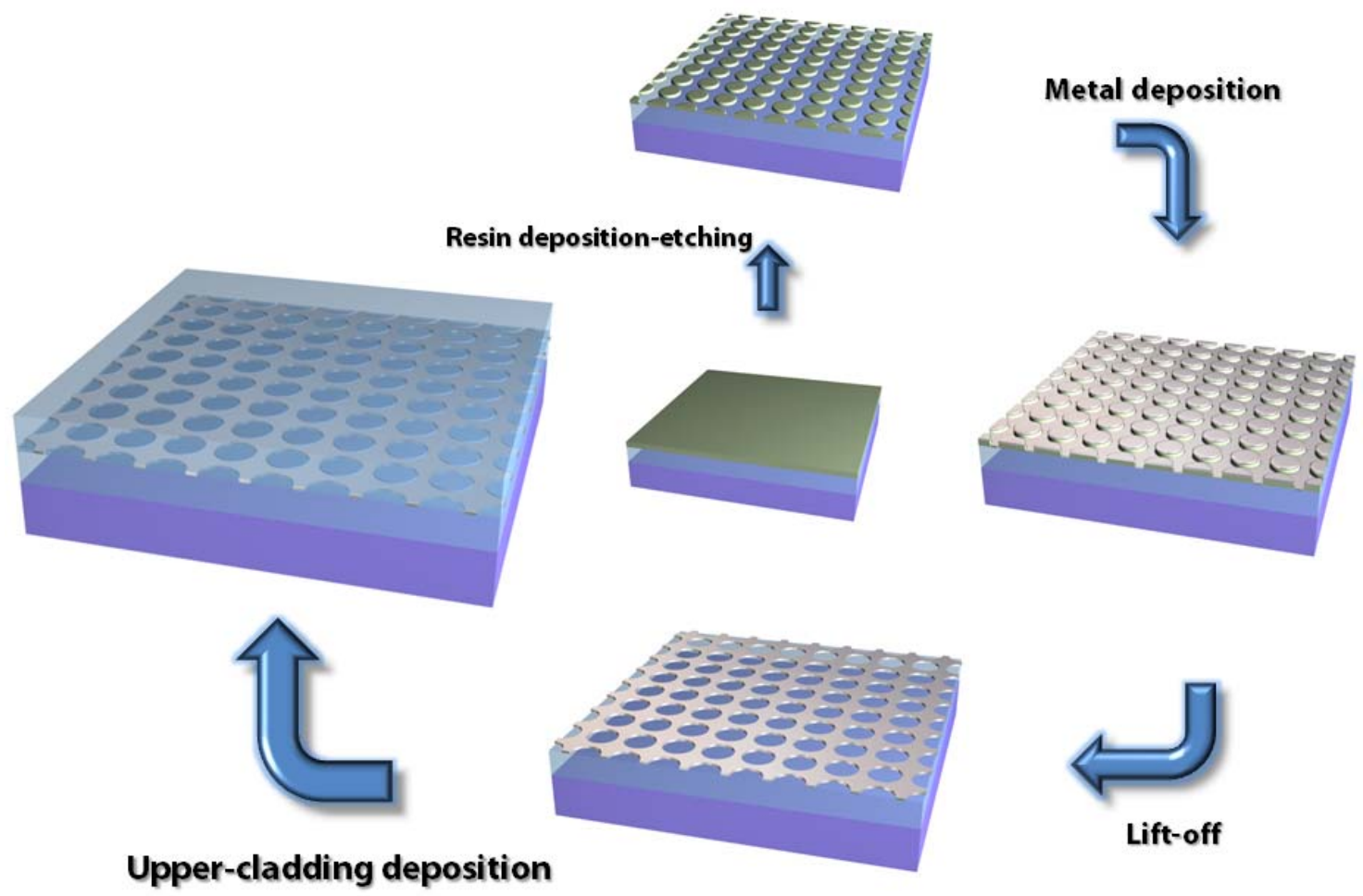

Figure 17. Schematic of the EOT filter fabrication process. A total of four manufacturing steps are needed in order to obtain a sandwiched hole array structure.

All the samples were optically characterized by means of transmission measurements made with a Fourier transform infrared (FTIR) spectroscopy system. The physical mechanism of the FTIR basically consists of a Michelson interferometer, as depicted in the sketch of the FTIR setup of Fig. 3. A Globar MIR source beam is divided in a $\mathrm{KBr}$ beamsplitter. Each beam is then reflected in a fixed or movable mirror, and the beams then recombine as they go through the beamsplitter, to form the interferogram signal. This signal then illuminates the fabricated subwavelength structures and transmitted light is collected with an optical microscope with a 0.4 numerical aperture coupled to the FTIR spectrometer to measure the transmission spectra of small samples areas. The signal in the microscope is detected with a liquid nitrogencooled mercurycadmium-telluride (MCT) detector. Finally, a Fourier transform is used to produce the transmission spectrum of the sample from the interferogram detected signal. A photograph of the FTIR installed at NTC facilities is provided at Fig. 18. 


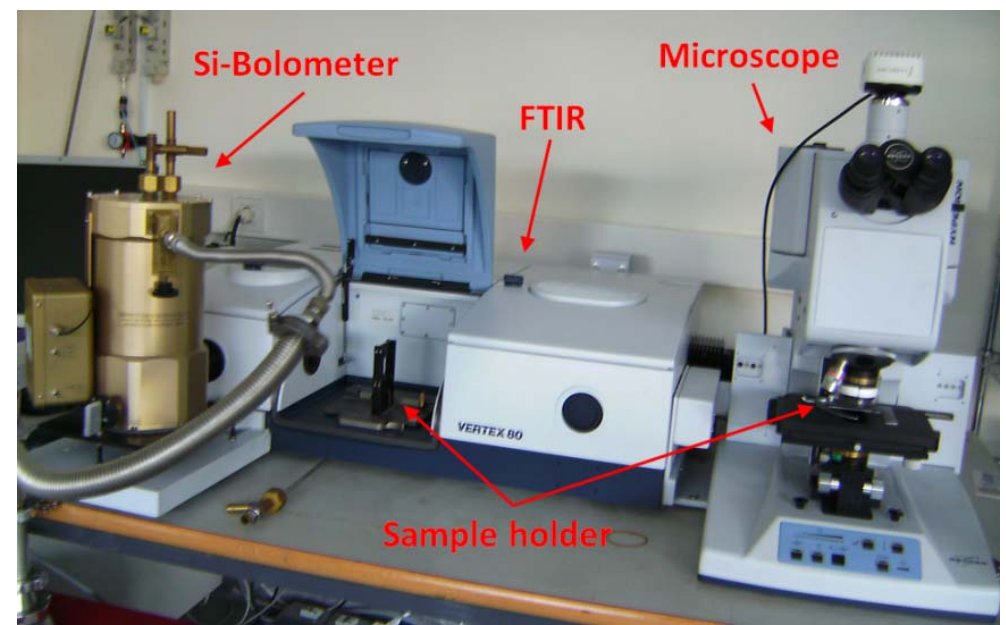

Figure 18. FTIR with microscope and Si-Bolometer equipment at NTC laboratory facilities.

\subsection{Experimental and numerical results}

Figure 4 shows SEM images of a group of $200 \times 200 \mu m^{2}$ area samples consisting of subwavelength hole arrays, with a square lattice array spacing of $a=2 \mu \mathrm{m}$, evaporated directly on a silicon wafer. In each sample the hole area differs in order to evaluate its influence on the EOT features and quality factor.

Their measured transmission spectra (dashed lines) are compared to simulations results (solid curves) in Fig. 5. An EOT peak is observed at around $8 \mu \mathrm{m}$ with a transmission level of $45 \%$, which is larger than the fractional area occupied by the holes and estimated to be $f=22 \%$. The minor discrepancies between simulations and measurements can be attributed to the fabrication process, that lead the actual hole dimensions vary slightly from the nominal values considered in the simulations. Leaving these discrepancies aside, it can be inferred that the transmission peaks occur at the particular wavelengths that satisfy the momentum conservation needed to excite SPP, and thus, are mainly controlled by the lattice spacing, as can be inferred from the closely spaced resonance peaks. However, the size of the holes causes slight deviations from the expected resonance wavelength. Likewise, the strength of the EOT is seen to depend on the hole size. It can be inferred that as the hole area increases, the resonance wavelength redshifts and the transmission enhances. On the other hand, the dip present at $7 \mu \mathrm{m}$ is due to Wood's anomaly for the first-order diffraction in the substrate [GHA-98].

To investigate the effect of the surrounding claddings on the EOT features, two set of samples were fabricated with SU8 cladding layers. One set comprises only one $4.4 \mu \mathrm{m}$-thick SU8 cladding layer below the gold film, whereas the gold film is surrounded by SU8 on both sides in the other set of samples. The area 
dimensions of $200 \times 200 \mu m^{2}$ and the square lattice array spacing of $a=2 \mu \mathrm{m}$ are maintained whilst varying the hole sizes in each sample.

The EOT spectra for both set of samples and its SEM images are shown in Fig. 6. By comparing the transmission spectra of Figs. 5 and 6, the presence of the SU8 cladding layers blueshifts the main characteristics of the EOT because of the lower SU8 refractive index than that of silicon. These results support the interpretation of EOT mediated by SPP excitation. Also Fabry-Perot peaks are clearly observed at shorter wavelengths due to the multilayer structure in Fig. 6 for both set of samples along with a shift in the EOT peaks of the sample with the upper SU8 cladding with respect to the samples with only the lower SU8 cladding. Another interesting observation is that when the upper SU8 cladding is present, the filter with smaller hole openings shows equal relative transmission as the filter with larger holes, but with a higher $Q$ value. This is of great relevance for filter design.

Finally, to prove the influence of lattice spacing on EOT features, a further sample was made with a lattice constant of $a=5 \mu \mathrm{m}$ and two SU8 claddings surrounding the gold film. Figure 7 depicts the EOT spectra and SEM images of two samples which differ in their hole openings. Once again, multiple FabryPerot peaks appear at shorter wavelengths due to the multilayer structure. As expected, the resonance wavelength has shifted to around $11 \mu \mathrm{m}$ due to the increase in lattice spacing. This confirms the theory that the EOT peak position is controlled by both the lattice spacing and dielectric constant of the cladding surrounding the metal layer in accordance with SPP theory. In addition, as the difference in hole size is small no drastic difference in EOT spectra between filters is measured.

All the characterized samples were made in order to investigate their principal transmission features with the intention of using the subwavelength hole array for filter design in the MIR region. Two of the most important parameters defining a filtering device are the resonance wavelength, and the quality factor. With the aim of finding how these two parameters vary with the geometrical parameters, the trend of the resonance wavelength and the quality factor versus the fractional open area of the films, i.e., the ratio between the unit cell area and the hole area are shown in Fig. 8.

The flat lines on Fig. 8(b) indicate that changes in the hole area cause almost no variations in the resonance wavelength, confirming that the resonance wavelength is practically independent of the hole size and varies mainly with the lattice and dielectric cladding parameters according SPP theory. Regarding the quality factor depicted in Fig. 8(a), a smaller hole area gives rise to a noticeable increase in the quality factor in all the samples at the expense of reduced 
transmission. Therefore, for practical purposes, the filter design will generally be a trade-off between the transmission level and the quality factor goal specifications. Also, by comparing samples with single SU8 substrate and the ones with double SU8 substrate in the same fractional area, it can be inferred that a higher quality factor value can be achieved when the subwavelength metal film has the same cladding on both sides.

All the transmission spectra measurements confirm the influence of hole size, lattice constant, and dielectric cladding on the role played by SPP excitation in EOT features. For filter design purposes, the data show that to achieve a specific resonance wavelength, it is mainly the lattice constant and dielectric claddings that must be taken into account. Hole shape and size are found to be more important when adjusting the resulting quality factor. Although the achieved quality factor values are lower than those provided by other technologies, these subwavelength structures have proven their function as extremely compact (in the direction of propagation) filter devices that allow their spectral features to be easily tuned and scale.

Although not showed in the publication, double metal layer structures were also fabricated. A SEM image of the structure along with its spectral response compared to a single metal layer structure are shown in Fig. 19. By looking carefully at the apertures of the SEM image, the lower metal layer can be noticed. Due to the fabrication process in which each metal layer is patterned separately, the alignment between layers is not perfect, diminishing the spectral response of the filter.
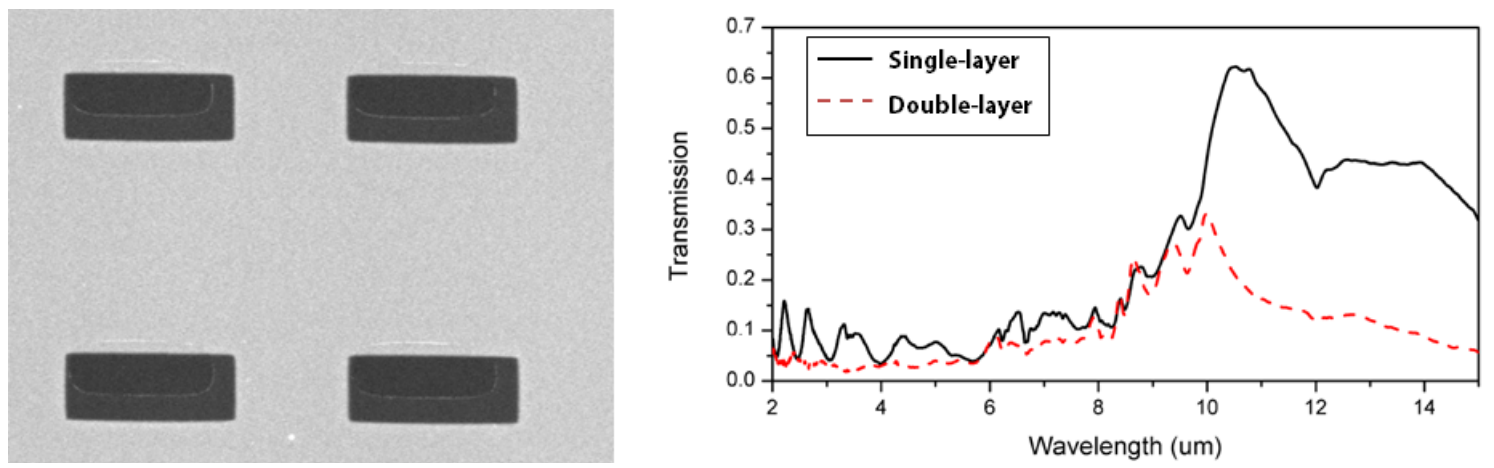

Figure 19. (left) SEM image of a doble metal layer EOT structure. (right) Spectral responses of single and double metal layer EOT structures.

\subsection{Conclusions}

In this chapter, several subwavelength hole array filters based on SPP excitation have been characterized, showing EOT resonant peaks in the MIR region. This behavior can be used to implement ultracompact optical filters in the MIR region for applications such as gas detection. The structure parameters effects on the 
resonance wavelength and quality factor is also studied. Showing that the resonance wavelength is mainly controlled by the lattice spacing and the dielectric constant of the surrounding claddings. Also, an increase of the hole area causes a transmission enhancement, a slight redshift in the resonances, and a lower quality factor.

In conclusion, hole openings on a subwavelength structured metallic film have a dramatic effect on the EOT phenomenon caused by SPP excitation. The simple SPP model relying on hole periodicity provides an excellent insight into EOT phenomenon and enables the approximate calculation of transmission peaks. However, EOT is a complex phenomenon - where not only the periodicity but also the hole characteristics must be considered when tailoring the structures for a given purpose. Hence, taking into account the hole size and shape as additional design parameters will enable a proper understanding and control of the EOT phenomenon. This, in turn, will extend the potential of these structures for use in developing modern applications, such as sensing. 


\title{
Midinfrared filters based on extraordinary optical transmission through subwavelength structured gold films
}

\author{
R. Ortuño, ${ }^{1, a)}$ C. García-Meca, ${ }^{1}$ F. J. Rodríguez-Fortuño, ${ }_{1}^{1}$ A. Håkansson, ${ }^{2}$ A. Griol, ${ }^{1}$ \\ J. Hurtado, ${ }^{1}$ J. A. Ayucar, ${ }^{1}$ L. Bellieres, ${ }^{1}$ P. J. Rodríguez, ${ }^{1}$ F. López-Royo, ${ }^{1}$ J. Martí, ${ }^{1}$ and \\ A. Martínez \\ ${ }^{1}$ Valencia Nanophotonics Technology Center, Universidad Politécnica de Valencia, Valencia 46022, Spain \\ ${ }^{2}$ DAS Photonics, Universidad Politécnica de Valencia, Valencia 46022, Spain
}

(Received 14 July 2009; accepted 11 November 2009; published online 23 December 2009)

\begin{abstract}
An experimental study is made of the enhanced optical transmission of nanostructured gold films in the midinfrared region. Results indicate that the excitation of surface plasmon polaritons due to periodicity plays a fundamental role in producing extraordinary optical transmission. The influence of the surrounding claddings, hole shape, and periodicity on the resonance wavelength and the quality factor is investigated. The aim is to use the subwavelength structures as ultracompact optical filters whose spectral features can be easily tuned and scaled. For filter design purposes, the results show that the main parameters affecting the resonance wavelength are the lattice constant and dielectric cladding. The hole shape and size are found to cause transmission enhancement and there is only a small resonance redshift when the hole area is increased. However, a lower quality factor is achieved when the hole area is increased. (C) 2009 American Institute of Physics.

[doi:10.1063/1.3272716]
\end{abstract}

\section{INTRODUCTION}

Over recent decades we have witnessed a surge of research and development activity related to surface plasmon polariton (SPP) based structures and devices. One of the initial scientific studies of SPPs dates back to the beginning of the twentieth century when Wood ${ }^{1}$ observed anomalous diffraction features in optical reflection measurements on metallic gratings. However, it was nearly seventy years after Wood's observations when Ritchie et $a l^{2}{ }^{2}$ described the anomalous behavior of metal gratings in terms of SPP resonances excited on the gratings after predicting theoretically that plasmon modes can exist near the surface of metals. ${ }^{3}$ In the late sixties, the optical excitation of SPPs using attenuated total reflection was demonstrated by Krestschmann and Raether ${ }^{4}$ and Otto; ${ }^{5}$ and their work made experiments on SPPs easily accessible to many researchers. SPPs have since been intensively studied and their main properties assessed. ${ }^{6,7}$ Their unique properties enable a wide range of practical applications, including gas detection and biosensing. ${ }^{8-10}$ In addition, the recent observation of extraordinary optical transmission (EOT) phenomenon through subwavelength apertures ${ }^{11}$ has further increased scientific interest in SPPs.

Since EOT was reported, subwavelength hole array structures periodically patterned on a metal film have attracted great attention owing to both its intriguing physical properties, in apparent contradiction with standard aperture theory, ${ }^{12}$ and its technological potential in areas such as light manipulation at nanoscale, optical resolution below the diffraction limit and highly compact optical filters. EOT physical interpretation was originally attributed to the excitation of SPPs on diffractive metallic screens based on the plasma-

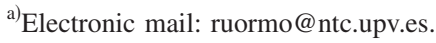

like behavior of metals at optical frequencies. ${ }^{11,13-15}$ However, EOT has been observed at frequencies where metals can no longer be considered as solid plasmas but rather as quasiperfect conductors that cannot sustain SPP excitation. ${ }^{16}$ These observations have opened the way for different theories in which SPP excitation is not the prime mechanism responsible for EOT. Suggestions to explain EOT have included diffraction mechanisms that emphasize the role of screen periodicity. ${ }^{17-20}$

An open debate about the physical origin of EOT still exists. This controversy enables EOT phenomenon to be addressed from many different points of view. Nevertheless, it is now widely accepted that EOT is related to the existence of surface waves supported by perforated metallic films and diffraction modes generated by periodic arrays of holes. In the optical regime, surface waves are SPPs; whereas in the microwave and terahertz frequencies the surface waves are supported by interactions between holes-even when metals behave as perfect conductors. Furthermore, at the perfect conductor limit the SPP concept can still be applied thanks to plasmonlike surface wave. This is the so-called 'spoof' plasmon, which is supported by structured metallic surfaces. ${ }^{21}$ The important role played by the SPP-like surface wave for EOT in the terahertz region was confirmed by several other experimental studies. ${ }^{22-25}$

Excitation of localized SPPs, ${ }^{26,27}$ as well as other mechanisms, ${ }^{28-30}$ can also play a role in EOT phenomena, although the main factor remains SPP excitation due to grating momentum. The prime role of grating momentum in EOT has also been demonstrated in stacked subwavelength arrays. ${ }^{25,31-38}$ In such configurations, the excitation of internal SPPs among metallic layers also contributes to the onset of EOT. ${ }^{31,38}$ One interesting feature of stacked arrays is that 

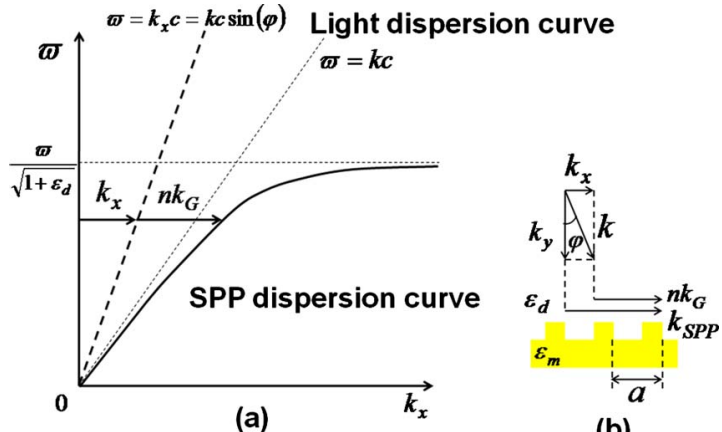

(b)

FIG. 1. (Color online) Coupling of light to a SPP wave using a grating: (a) dispersion of light and SPP waves. To allow light to couple to SPPs the extra momentum $n k_{G}$ is needed to increase the incident light momentum $k_{x}$; (b) wave vector diagram of light to SPP coupling. The extra momentum $n k_{G}$ is achieved thanks to the periodic patterned surface.

a transmission with an effective negative index of refraction can be achieved, ${ }^{31,35-39}$ thereby connecting the worlds of plasmonics and metamaterials.

In this work we present experimental measurements of EOT in the midinfrared range (MIR) of several fabricated subwavelength structures. The measured transmission spectrum shows how the grating momentum appears to define the main resonances of the spectrum. This confirms that EOT features are mainly due to the resonant excitation of SPPs by incident light. The simplicity with which the spectral features can be tuned and scaled has generated considerable interest and promoted subwavelength structures as a core element of modern optical devices. In this sense, one of our major aims is to design ultracompact optical filters in the MIR region. Several samples with various hole sizes and shapes with surrounding claddings and lattice periodicity were made in order to investigate their influence on the main characteristics defining a filter: i.e., the resonance wavelength $\lambda_{0}$; and the quality factor, defined as $Q=\lambda_{0} / \Delta \lambda$, where $\Delta \lambda$ is the full width at half maximum of the transmission resonance.

\section{SPP RESONANCE}

SPP resonance is a charge-density oscillation that can exist at the interface between two media with dielectric constants of opposite signs, for instance, a metal and a dielectric. The corresponding propagation constant $k_{\mathrm{SPP}}$ of the SPP, propagating at the interface between a semi-infinite dielectric and a smooth metal, can be found by requiring the continuity of the tangential electric and magnetic field components across the interface, and can be written as

$$
k_{\mathrm{SPP}}=k_{0} \sqrt{\frac{\varepsilon_{m} \varepsilon_{d}}{\varepsilon_{m}+\varepsilon_{d}}},
$$

where $k_{0}=2 \pi / \lambda$ denotes the free space wave number, and $\varepsilon_{m}$ and $\varepsilon_{d}$ are the complex relative dielectric constants of the metal and dielectric layer, respectively.

As follows from Eq. (1), the SPP propagation constant $k_{\mathrm{SPP}}$ at a planar metal-dielectric interface is always larger than that of light $k_{0}$ for any given frequency, thus preventing its excitation by any incident radiation. This wave vector mismatch is represented in Fig. 1(a). An additional momen-

tum needs to be added to the momentum of the incident optical wave to match that of the SPPs. Consequently, the coupling of light to SPPs can rely on light scattering at periodic patterned surfaces, which provides the incident wave with an additional momentum multiple of $k_{G}=2 \pi / a$, where $a$ is the grating period.

Figure 1(b) shows a vector diagram of the coupling process for a grating interface formed between a metal and a dielectric with dielectric parameters $\varepsilon_{m}$ and $\varepsilon_{d}$, respectively. If light with a wave vector $k=2 \pi / \lambda_{d}$, with $\lambda_{d}=\lambda / \sqrt{\varepsilon_{d}}$ being the wavelength of light in the dielectric adjacent to the metal, illuminates a grating at an angle of incidence $\varphi$, then the wave vector matching condition in the $x$ direction is

$$
\frac{2 \pi}{\lambda_{d}} \sin (\varphi)+n \frac{2 \pi}{a}=k_{\mathrm{SPP}}
$$

and $n$ is an integer. Equation (2) for wave vector matchingwhich can be easily extended for two-dimensional gratingsestablishes strict relationships between the wavelength of light in the dielectric $\lambda_{d}$, the angle of incidence $\varphi$, and the grating period $a$. If the grating is formed by milling subwavelength holes through the metal film, the transmission spectra of such arrays are characterized by resonance peaks in which Eq. (2) is fulfilled (or rather its two-dimensional equivalent) and characterized by a $(n, m)$ scattering order of the array giving rise to the EOT ( $m$ being an integer for the case of two-dimensional gratings). This is true even in the case of optically thick metals. ${ }^{11}$ For the case of normal incidence, the wavelengths for these resonances according to Eqs. (1) and (2), are given by

$$
\lambda=\frac{a}{n^{2}+m^{2}} \sqrt{\frac{\varepsilon_{m} \varepsilon_{d}}{\varepsilon_{m}+\varepsilon_{d}}} .
$$

This expression is approximate because Eqs. (1) and (2) do not take into account the presence of hole and scattering losses. Nevertheless, Eq. (3) provides a simple means to label and associate the resonances with the corresponding $(n, m)$ scattering order without requiring the use of more complex methods that consider the scattering of the holes. ${ }^{13,40,41}$

\section{FABRICATION}

Several subwavelength hole arrays with different hole shapes and periods were made by vacuum evaporation of a thin gold film with a thickness of $100 \mathrm{~nm}$ on a transparent silicon substrate wafer for characterization in the MIR region. The nanostructured gold films were surrounded by SU8 claddings on both sides to optimize their transmission efficiency. ${ }^{40}$ In addition, structures in which the gold film was fabricated directly over the Si wafer, or with only one SU8 cladding, were also characterized to measure the cladding influence on the transmission spectrum. Among the experimental methods for structuring these thin films laterally at microscale and nanoscale, electron-beam lithography was used as it has proven to be the most versatile. This is due to its flexibility and the availability of a variety of platforms, usually based on scanning electron microscopes (SEMs). 


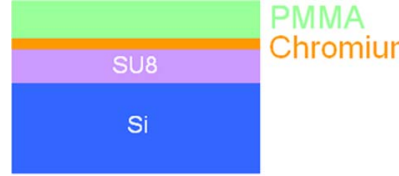

(a) Spin-coating

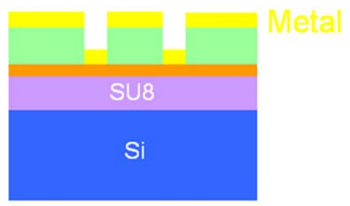

(c) Metal deposition
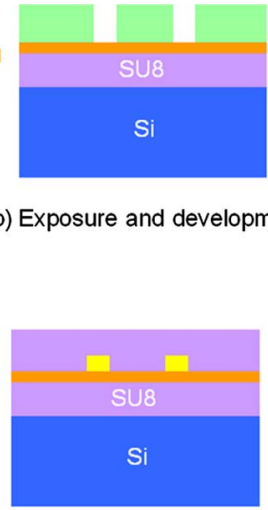

(d) Lift-off and spin-coating (b) Exposure and development

FIG. 2. (Color online) Fabrication flowchart. The main fabrication steps are: (a) spin-coating, (b) exposure and development to transfer the sample pattern, (c) metal deposition by a sputtering mechanism, and finally (d) lift-off and spin-coating to achieve the desired periodic metallic structure.

Figure 2 shows the schematics of the fabrication process for the subwavelength metallic array structures embedded in SU8 claddings. A clean silicon wafer was spin-coated with a 4.4- $\mu \mathrm{m}$-thick SU8 polymer layer and then with a $5 \mathrm{~nm}$ chromium layer, which was used to provide adhesion for the gold film. A layer of electron-sensitive resist polymethyl methacrylate (PMMA) was deposited on the substrate by spincoating, as shown in Fig. 2(a). The sample pattern was then transferred to the electron resist via exposure to a focused electron beam and various periodic patterns can then be transferred simply by changing the dose rate. Subsequent chemical development removed the resist from all the regions exposed to the electron beam as schematically illustrated in Fig. 2(b). Gold metal was then deposited on the sample by a sputtering process as shown in Fig. 2(c). Following deposition of the thin gold film, a chemical lift-off process removed the PMMA resist layer from the chromium surface. This left the periodic metallic structures deposited directly on the chromium-SU8 substrate through the openings in the resist mask. Finally, the spin-coating of a top cladding of another $4.4-\mu \mathrm{m}$-thick SU8 layer was performed. Since the symmetry of the structure is very important for the transmission properties, we carefully ensured that the cladding layers had the same refractive index. This was guaranteed by applying the same SU8 polymer for the top and bottom claddings; and using identical spinning and curing conditions. After the final SU8 polymer curing, the wafer shown in Fig. 2(d) was ready for optical characterization.

The fabrication process for structures with only one SU8 cladding is identical to that previously described but without the last spin-coating deposition of the upper SU8 polymer cladding. Obviously, the bottom and upper SU8 polymer claddings deposition processes are not carried out for structures made directly on the silicon wafer.

\section{CHARACTERIZATION}

For optical characterization of the samples various transmission measurements were made using a Bruker Vertex 80 Fourier transform infrared (FTIR) spectroscopy system.

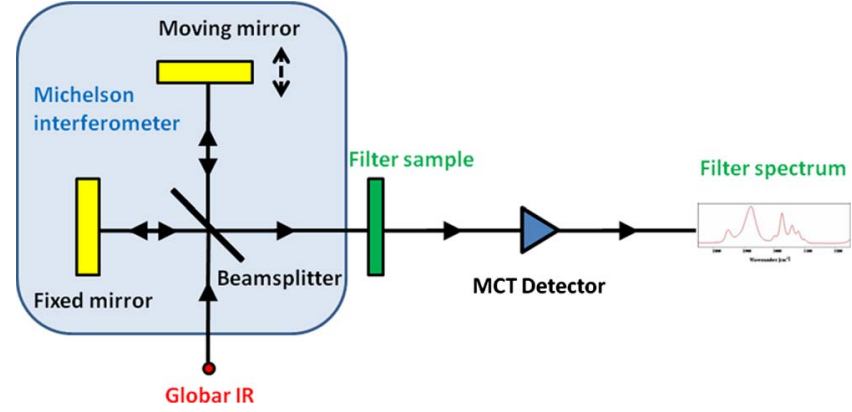

FIG. 3. (Color online) Sketch of the measurement setup. The Michelson interferometer and the Globar source represent, in a simplified way, the internal mechanism of the FTIR. The optical microscope is represented by the MCT detector block.

The physical mechanism of the FTIR basically consists of a Michelson interferometer, as schematically depicted in the sketch of the setup in Fig. 3. A Globar MIR source beam is divided in a $\mathrm{KBr}$ beamsplitter. Each beam is then reflected in a fixed or movable mirror, and the beams then recombine as they go through the beamsplitter, to form the interferogram signal. This signal then illuminates the fabricated subwavelength structures and transmitted light is collected with an optical microscope (Bruker Hyperion) with a $15 \times$ objective ( 0.4 numerical aperture) coupled to the FTIR spectrometer to measure the transmission spectra of the samples. The signal in the microscope is detected with a liquid nitrogencooled mercury-cadmium-telluride (MCT) detector. Finally, a Fourier transform is used to produce the transmission spectrum of the sample from the interferogram transmitted signal.

\section{EXPERIMENTAL RESULTS}

In this section we present the experimental results obtained in the characterization of the subwavelength hole arrays in the MIR region.

An initial group A of three samples A1, A2, and A3 was made. Figure 4 shows SEM images of samples consisting of subwavelength hole arrays made with 100-nm-thick gold films evaporated on a silicon wafer with an adhesive $5 \mathrm{~nm}$ chromium layer. Several samples with different hole sizes and shapes were made to evaluate the influence of the hole on the EOT features and quality factor $Q$. The corresponding microaperture dimensions of the samples shown in the SEM images are $1.63 \times 0.78,1.36 \times 0.76$, and $1.08 \times 0.70 \mu \mathrm{m}^{2}$ for samples A1, A2, and A3, respectively. All samples were made on the same chip with equal area dimensions of 200 $\times 200 \mu \mathrm{m}^{2}$ and a square lattice array spacing of $a=2 \mu \mathrm{m}$.

Transmission spectra were recorded using a Bruker FTIR spectrometer with the light source incident from the silicon substrate side. Figure 5 shows both measured (dashed curves) and simulated (solid curves) EOT spectra of the periodic subwavelength arrays of sample A. A peak is observed at around $8 \mu \mathrm{m}$ with a transmission level of $45 \%$, which is larger than $f$, the fractional area occupied by the holes and estimated to be $f \approx 22 \%$. Simulations were performed with CST MICROWAVE STUDIO software and the frequency dependent dielectric constant of the gold layer was described using the Drude model 

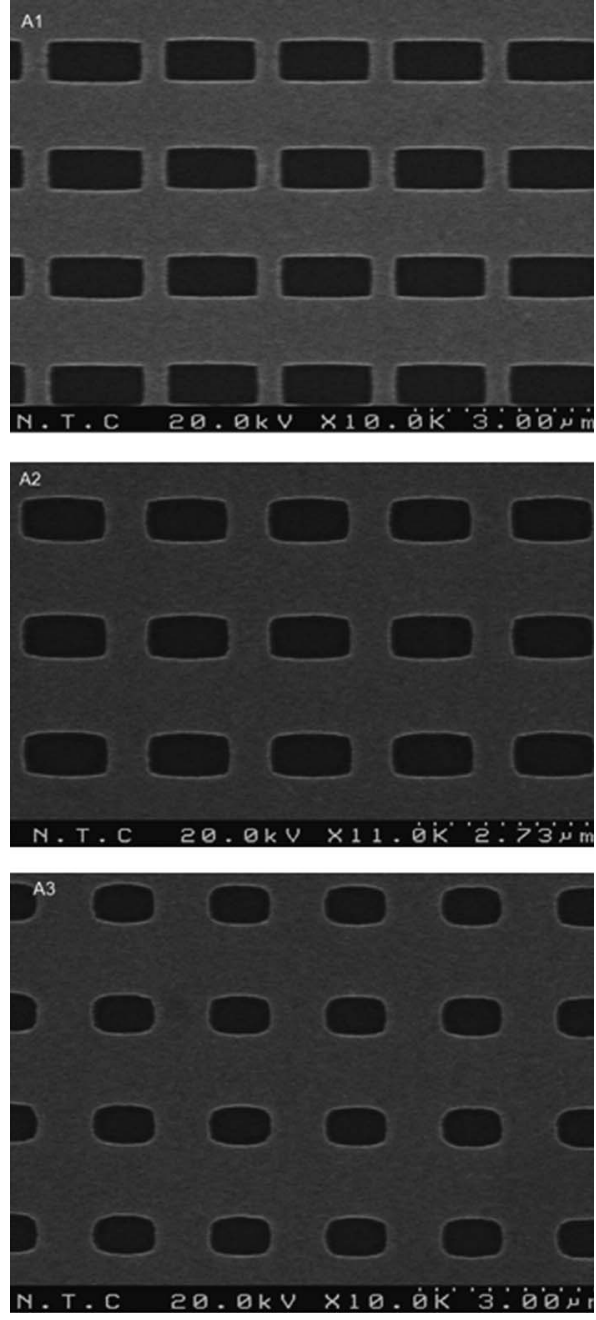

FIG. 4. SEM images of subwavelength periodic arrays of sample A made with $100 \mathrm{~nm}$ of gold film evaporated on a silicon wafer. Different hole openings are made: (top) A1 with hole dimensions $1.63 \times 0.78 \mu \mathrm{m}^{2}$, (middle) A2 with dimensions $1.36 \times 0.76 \mu \mathrm{m}^{2}$, and (down) A3 with 1.08 $\times 0.70 \mu \mathrm{m}^{2}$. All samples have a square lattice array spacing of $a=2 \mu \mathrm{m}$.

$$
\varepsilon=\varepsilon_{\infty}-\frac{\varpi_{p}^{2}}{\varpi^{2}+i \gamma \varpi}
$$

using the parameters $\varepsilon_{\infty}=9$, a plasma frequency $\varpi_{p}$ $=1.3676 \cdot 10^{16} \mathrm{rad} / \mathrm{s}$, and a collision frequency $\gamma=1.0027$ $\times 10^{14} \mathrm{~Hz}$ extracted from Ref. 41. For silicon, a dielectric constant of $\varepsilon_{\mathrm{Si}}=12.1104$ was considered, whereas the effect of the thin chromium layer was neglected in the simulations.

The resonance wavelengths predicted by Eq. (3) do not exactly match the simulations and measurements because Eq. (3) does not take into account the presence of holes and their associated scattering losses and so the actual resonant peaks always appear redshifted from the expected resonance wavelength. In addition, the discrepancies between simulations and measurements can be attributed to the fabrication process, e.g., the actual hole dimensions vary slightly from the nominal values considered in the simulations-which are $1.5 \times 0.7,1.3 \times 0.7$, and $1.1 \times 0.70 \mu \mathrm{m}^{2}$ for samples A1, A2, and $\mathrm{A} 3$, respectively.

Leaving these discrepancies aside, from Fig. 5 it can be inferred that the transmission peaks approximately occur at

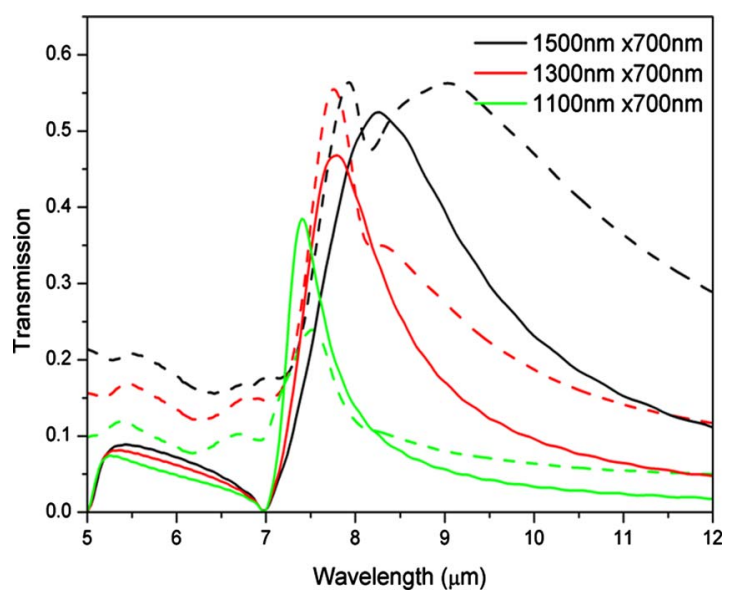

FIG. 5. (Color online) EOT measured (dashed) and simulated (solid) transmission spectra for samples (black) A1, (red) A2, and (green) A3. The transmission peak at around $8 \mu \mathrm{m}$ is due to SPP excitation and can be mainly controlled by the lattice spacing. A redshift in the resonant wavelength and a transmission enhancement can be seen as the hole area increases. The dip at $7 \mu \mathrm{m}$ is due to Wood's anomaly.

the particular wavelengths that satisfy the momentum conservation given by Eq. (3), and thus, are mainly controlled by the lattice spacing $a$, as can be inferred from the closely spaced resonance peaks. Moreover, the size of the holes also causes slight deviations from the expected resonance wavelength. Likewise, the strength of the EOT is seen to depend on the hole size. It can be inferred that as the hole area increases, the resonance wavelength redshifts and the transmission is enhanced.

The dip present at $7 \mu \mathrm{m}$ is due to Wood's anomaly for the first-order diffraction in the substrate, which is approximately at $n_{\mathrm{Si}} a$, where $n_{\mathrm{Si}}=\sqrt{\varepsilon_{\mathrm{Si}}}$ is the refractive index of the silicon substrate. ${ }^{13}$

To investigate the effect of the surrounding claddings on the EOT features, groups of samples B and C were fabricated with SU8 cladding layers. Sample B differs from sample A in that $90 \mathrm{~nm}$ of gold was deposited on top of a $4.4-\mu \mathrm{m}$-thick SU8 cladding layer. For sample C, a further $4.4-\mu \mathrm{m}$-thick SU8 cladding layer was deposited on top of the gold film. An adhesive chromium layer was also deposited to increase adhesion between the lower SU8 cladding and gold film in both samples. Thus, sample B comprises only one SU8 cladding layer below the gold layer, whereas the gold layer is surrounded by SU8 on both sides in sample C. A silicon wafer was used as a substrate in both samples in the same way as sample A-using area dimensions of $200 \times 200 \mu \mathrm{m}^{2}$ and a square lattice array spacing of $a=2 \mu \mathrm{m}$.

As previously, several designs with different subwavelength hole sizes were made for each sample. For comparison, Fig. 6 shows the EOT spectra for samples B and C with microapertures of sizes $1.6 \times 0.7$ and $1.38 \times 0.7 \mu \mathrm{m}^{2}$ and whose SEM images (I) and (II) are inset, respectively.

By comparing the transmission spectra of Figs. 5 and 6 it can be seen that due to the presence of the SU8 cladding layer the main characteristics of the EOT have shifted to shorter wavelengths because the SU8 refractive index $n_{\mathrm{SU} 8}$, which has been characterized as having a value of $n_{\mathrm{SU} 8}=2$, is lower than that of silicon. These results support the interpre- 


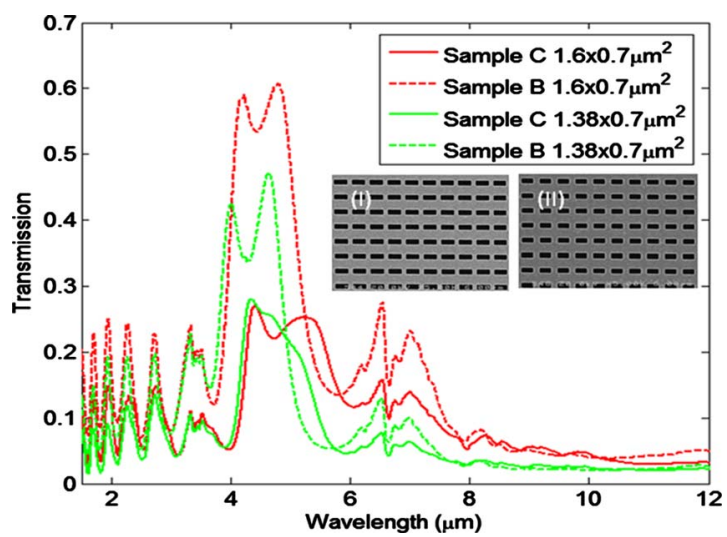

FIG. 6. (Color online) Measured EOT transmission spectra for sample B (single SU8 cladding) and C (double SU8 cladding). Because of the presence of the SU8 claddings, the resonance has shifted to shorter wavelengths. (Inset) SEM images of the characterized filters with microaperture sizes of (I) $1.6 \times 0.7 \mu \mathrm{m}^{2}$, and (II) $1.38 \times 0.7 \mu \mathrm{m}^{2}$. The lattice spacing is kept to $a=2 \mu \mathrm{m}$

tation of EOT mediated by SPP excitation. Another feature in samples B and C are the Fabry-Perot peaks clearly observed at shorter wavelengths due to the multilayer structure.

From the EOT spectra of samples $\mathrm{B}$ and $\mathrm{C}$ a shift can definitely be observed in the EOT peaks after the deposition of the upper SU8 cladding. Another interesting observation is that when the upper SU8 cladding is present (sample C), the filter with smaller hole openings shows equal relative transmission when compared with the filter that has larger holes; however, it also has a higher $Q$ value-as will be shown. This is of great relevance for filter design.

Finally, to prove the influence of lattice spacing $a$ on EOT features, a further sample D was made. Sample D also has two SU8 claddings surrounding the gold film, and differs from sample $\mathrm{C}$ only in the lattice spacing of the gold film subwavelength apertures. A lattice constant of $a=5 \mu \mathrm{m}$ was chosen for sample D instead of the previous $2 \mu \mathrm{m}$.

Figure 7 depicts the EOT spectra for two filters of sample D with hole openings of $2.88 \times 1.24$ and 2.88

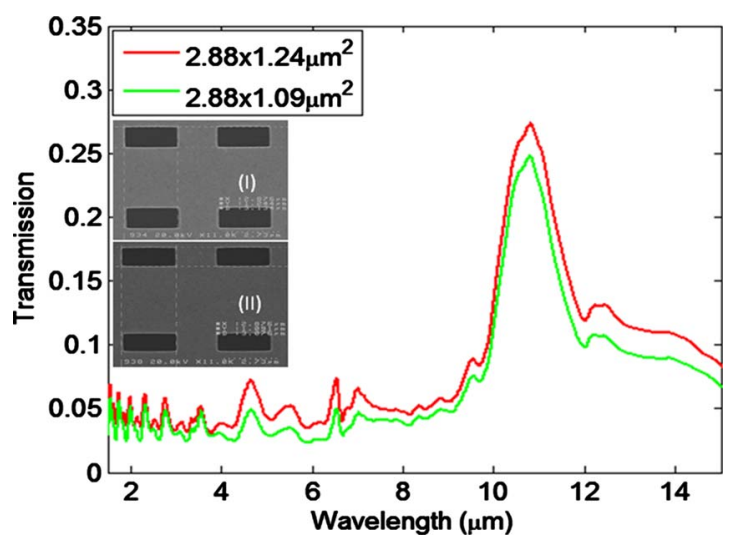

FIG. 7. (Color online) Measured EOT transmission spectra for sample D in which the lattice spacing is now $a=5 \mu \mathrm{m}$. The resonance appearing around $11 \mu \mathrm{m}$ confirms that its origin can be attributed to SPP excitation and its position can be mainly controlled by the lattice spacing and the dielectric constant of the surrounding claddings. (Inset) SEM images of the characterized filters with hole openings of (I) $2.88 \times 1.24 \mu \mathrm{m}^{2}$, and (II) 2.88 $\times 1.09 \mu \mathrm{m}^{2}$.

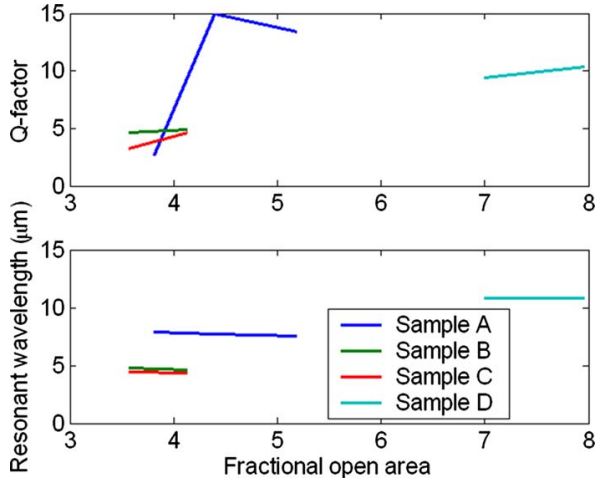

FIG. 8. (Color online) (up) Measured quality factor $Q$ and (down) resonant wavelength vs fractional open area for samples A, B, C, and D. It can be inferred that a smaller hole area gives rise to a higher quality factor $Q$. Comparing samples B (single SU8 cladding) and C (double SU8 cladding) the quality factor enhances when the sample has the same cladding on both sides. Also, it is seen how the resonance wavelength varies mainly with the lattice and dielectric cladding parameters, while showing slight variations with the hole area.

$\times 1.09 \mu \mathrm{m}^{2}$. Their SEM images are inset and labeled as (I) and (II), respectively. Once again, multiple Fabry-Perot peaks appear at shorter wavelengths due to the multilayer structure. As expected from Eq. (3), the resonance wavelength has shifted to around $11 \mu \mathrm{m}$ due to the increase in lattice spacing. This confirms the theory that the EOT peak position is controlled by both the lattice spacing and dielectric constant of the cladding surrounding the metal layer in accordance with Eq. (3). In addition, as the difference in hole size is small no drastic difference in EOT spectra between filters is measured.

All the characterized samples were made in order to investigate their principal transmission features with the intention of using the subwavelength hole array for filter design in the MIR region. Two of the most important parameters defining a filtering device are the resonance wavelength $\lambda_{0}$, and the quality factor $Q=\lambda_{0} / \Delta \lambda$. With the aim of finding how these two parameters vary with the geometrical parameters, we plot in Fig. 8 the trend of the $(1,0)$ resonance wavelength $\lambda_{0}$ and the quality factor $Q$ versus the fractional open area of the films, i.e., the ratio between the unit cell area and the hole area.

The flat lines on Fig. 8(b) indicate that changes in the hole area cause almost no variations in the resonance wavelength, confirming that the resonance wavelength is practically independent of the hole size and varies mainly with the lattice and dielectric cladding parameters according to Eq. (3). Regarding the quality factor, we can see in Fig. 8(a) that a smaller hole area gives rise to a noticeable increase in the quality factor $Q$ in all the samples-at the expense of reduced transmission. Therefore, for practical purposes, the filter design will generally be a trade-off between the transmission level and the quality factor goal specifications. Also, by comparing samples B (single SU8 substrate) and C (double SU8 substrate) in the same fractional area, it can be inferred that a higher quality factor value can be achieved when the subwavelength metal film has the same cladding on both sides.

The transmission spectra measurements for all the 
samples confirm the influence of hole size, lattice constant, and dielectric cladding on the role played by SPP excitation in EOT features. For filter design purposes, the data show that to achieve a specific resonance wavelength, it is mainly the lattice constant and dielectric claddings that must be taken into account. Hole shape and size are found to be more important when adjusting the resulting quality factor. Although the achieved quality factor values are lower than those provided by other technologies, these subwavelength structures have proven their function as extremely compact (in the direction of propagation) filter devices that allow their spectral features to be easily tuned and scaled.

\section{CONCLUSION}

Several subwavelength hole array filters based on SPP excitation have been made and characterized, showing EOT resonant peaks in the MIR region. This behavior can be used to implement ultracompact optical filters in the MIR region for applications such as gas detection. We have experimentally studied how the transmission properties (resonance wavelength and quality factor) are affected by structure parameters. It has been shown that the resonance wavelength is mainly controlled by the lattice spacing and the dielectric constant of the surrounding cladding. We have also found that increasing the hole area causes a transmission enhancement and a slight redshift in the resonances. However, a lower quality factor is achieved when increasing the hole area.

In conclusion, hole openings on a subwavelength structured metallic film have a dramatic effect on the EOT phenomenon caused by SPP excitation. The simple SPP model relying on hole periodicity provides an excellent insight into EOT phenomenon and enables the approximate calculation of transmission peaks. However, EOT is a complex phenomenon-where not only the periodicity but also the hole characteristics must be considered when tailoring the structures for a given purpose. Hence, taking into account the hole size and shape as additional design parameters will enable a proper understanding and control of the EOT phenomenon. This, in turn, will extend the potential of these structures for use in developing modern applications-such as sensing.

\section{ACKNOWLEDGMENTS}

Financial support from the Spanish Ministry of Science and Innovation (MICINN) under contracts CSD2008-00066, TEC2008-06871-C02-02, and PET2007-0505, and a FPI grant from the UPV are gratefully acknowledged.

${ }^{1}$ R. W. Wood, Philos. Mag. 4, 396 (1902).

${ }^{2}$ R. H. Ritchie, E. T. Arakawa, J. J. Cowan, and R. N. Hamm, Phys. Rev. Lett. 21, 1530 (1968).

${ }^{3}$ R. H. Ritchie, Phys. Rev. 106, 874 (1957).
${ }^{4}$ E. Kretschmann and H. Raether, Z. Naturforsch. B 23A, 2135 (1968).

${ }^{5}$ A. Otto, Z. Phys. 216, 398 (1968).

${ }^{6} \mathrm{H}$. Raether, Springer Tracts in Modern Physics, Surface Plasmons on Smooth and Rough Surfaces and on Gratings (Springer, Berlin, 1988).

A. D. Boardman, Electromagnetic Surface Modes (Wiley, New York, 1982).

${ }^{8}$ C. Nylander, B. Liedberg, and T. Lind, Sens. Actuators 3, 79 (1982).

${ }^{9}$ B. Liedberg, C. Nylander, and I. Lundström, Sens. Actuators 4, 299 (1983).

${ }^{10}$ B. Liedberg, C. Nylander, and I. Lundström, Biosens. Bioelectron. 10, i (1995).

${ }^{11}$ T. W. Ebbesen, H. J. Lezec, H. F. Ghaemi, T. Thio, and P. A. Wolf, Nature (London) 391, 667 (1998).

${ }^{12}$ H. A. Bethe, Phys. Rev. 66, 163 (1944).

${ }^{13}$ H. F. Ghaemi, T. Thio, D. E. Grupp, T. W. Ebbesen, and H. J. Lezec, Phys. Rev. B 58, 6779 (1998).

${ }^{14}$ D. E. Grupp, H. J. Lezec, T. W. Ebbesen, K. M. Pellerin, and T. Thio, Appl. Phys. Lett. 77, 1569 (2000).

${ }^{15}$ L. Martín-Moreno, F. J. García-Vidal, H. J. Lezec, K. M. Pellerin, T. Thio, D. E. Grupp, J. B. Pendry, and T. W. Ebbesen, Phys. Rev. Lett. 86, 1114 (2001).

${ }^{16}$ M. Beruete, M. Sorolla, I. Campillo, J. S. Dolado, L. Martín-Moreno, J. Bravo-Abad, and J. F. García-Vidal, Opt. Lett. 29, 2500 (2004).

${ }^{17}$ M. M. J. Treacy, Appl. Phys. Lett. 75, 606 (1999).

${ }^{18}$ Q. Cao and P. Lalanne, Phys. Rev. Lett. 88, 057403 (2002).

${ }^{19}$ H. J. Lezec and T. Thio, Opt. Express 12, 3629 (2004).

${ }^{20}$ F. J. García de Abajo, R. Gómez-Medina, and J. J. Sáenz, Phys. Rev. E 72, 016608 (2005).

${ }^{21}$ J. B. Pendry, L. Martín-Moreno, and F. J. García-Vidal, Science 305, 847 (2004).

${ }^{22}$ M. Tanaka, F. Miyamaru, M. Hangyo, T. Tanaka, M. Akazawa, and E. Sano, Opt. Lett. 30, 1210 (2005).

${ }^{23}$ A. P. Hibbins, M. J. Lockyear, I. R. Hooper, and J. R. Sambles, Phys. Rev. Lett. 96, 073904 (2006).

${ }^{24}$ H. Cao and A. Nahata, Opt. Express 12, 1004 (2004).

${ }^{25}$ F. Miyamaru and M. Hangyo, Phys. Rev. B 71, 165408 (2005).

${ }^{26}$ A. Degiron, H. J. Lezec, N. Yamamoto, and T. W. Ebbesen, Opt. Commun. 239, 61 (2004)

${ }^{27}$ K. L. van der Molen, K. J. Klein Koerkamp, S. Enoch, F. B. Segerink, N. F. van Hulst, and L. Kuipers, Phys. Rev. B 72, 045421 (2005).

${ }^{28}$ F. J. García de Abajo, Opt. Express 10, 1475 (2002).

${ }^{29}$ F. J. García-Vidal, E. Moreno, J. A. Porto, and L. Martín-Moreno, Phys. Rev. Lett. 95, 103901 (2005).

${ }^{30}$ F. J. García-Vidal, L. Martín-Moreno, E. Moreno, L. K. S. Kumar, and R. Gordon, Phys. Rev. B 74, 153411 (2006).

${ }^{31}$ R. Ortuño, C. García-Meca, F. J. Rodríguez-Fortuño, J. Martí, and A. Martínez, Phys. Rev. B 79, 075425 (2009).

${ }^{32}$ Y.-H. Ye and J.-Y. Zhang, Opt. Lett. 30, 1521 (2005).

${ }^{33}$ H. Li, S. Xie, R. Zhou, Q. Liu, X. Zhou, and M. Yuan, J. Phys.: Condens. Matter 20, 415223 (2008).

${ }^{34}$ Z. H. Tang, R. W. Peng, Z. Wang, X. Wu, Y. J. Bao, Q. J. Wang, Z. J. Zhang, W. H. Sun, and M. Wang, Phys. Rev. B 76, 195405 (2007).

${ }^{35}$ M. Beruete, M. Sorolla, and I. Campillo, Opt. Express 14, 5445 (2006).

${ }^{36}$ M. Beruete, M. Sorolla, M. Navarro-Cía, F. Falcone, I. Campillo, and V. Lomakin, Opt. Express 15, 1107 (2007).

${ }^{37}$ M. Navarro-Cía, M. Beruete, M. Sorolla, and I. Campillo, Opt. Express 16, 560 (2008).

${ }^{38}$ A. Mary, S. G. Rodrigo, F. J. García-Vidal, and L. Martín-Moreno, Phys. Rev. Lett. 101, 103902 (2008).

${ }^{39}$ C. García-Meca, R. Ortuño, F. J. Rodríguez-Fortuño, J. Martí, and A. Martínez, Opt. Express 17, 6026 (2009).

${ }^{40}$ A. Krishnan, T. Thio, T. J. Kim, H. J. Lezec, T. W. Ebbesen, P. A. Wolff, J. B. Pendry, L. Martín-Moreno, and F. J. García-Vidal, Opt. Commun. 200, 1 (2001).

${ }^{41}$ A. D. Rakic, A. B. Djurisic, J. M. Elazar, and M. L. Majewski, Appl. Opt. 37, 5271 (1998). 



\section{Chapter 7}

\section{General conclusions and future work}

This chapter contains the main conclusions elicited throughout the Thesis along with those susceptible facets to be explored in the future.

\subsection{General conclusions}

In this thesis experimental and numerical results have been presented related to EOT achieved through SPP excitation in nanostructured metallic sheets patterned with subwavelength apertures arrays. Additionally to EOT phenomenon, an artificial effective magnetic activity, which paves the way to achieve a negative refractive index, is also encountered in stacked subwavelength aperture arrays. The underlying physics of the magnetic dipole appearing in double-layer metallic structures is investigated showing its relationship with the excitation of SPP, connecting the world of plasmonics with that of metamaterials. From the analytically calculated SPP dispersion relation, the light coupling to external and internal SPP modes leads to the onset of EOT, as numerically proved with a great accuracy. In addition, at the internal-SPP resonant frequencies the negative effective permeability is achieved as a VCL is formed between the metallic layers. The model presented is intended to facilitate the interpretation and design of stacked subwavelength structures, such as the fishnet metamaterial, which can be used to obtain negative-index metamaterials showing EOT.

Exploiting the close relationship between internal SPP and the artificial magnetic response in fishnet structures, an alternative and straightforward approach to achieve a NIM over a broad spectral bandwidth is presented. The proposed metamaterial unit cell consists of stacking fishnet structures with different dielectric thickness, carefully selected so as to excite internal SPP at 
close enough frequencies. This frequency proximity provokes hybridization effects on the SPP resonances in the cascaded fishnet metamaterial that, consequently, leads to broaden the magnetic resonant response that, in combination with a negative permittivity, can cause a broad negative refractive index response. The suitability of the design to build a "bulk" NIM over a broad spectral bandwidth that could be employed to develop device applications is checked with the convergence of the optical metamaterial effective parameters.

In addition to the EOT phenomenon related to SPP excitation, multiple EOT peaks appear in the transmission spectrum of light through nanostructured embedded metallic films, a phenomenon independent of conventional tunnelling transmission. It can be inferred from the performed theoretical and numerical analysis that these resonances appear only in the case of periodically patterned metal films due to the coupling of light to Fabry-Perot modes in the claddings evanescently coupled through the drilled metallic film. Consequently, these results are of great significance as they relate the important role of the periodicity not only to surface modes, like SPPs, but also to propagating modes in the dielectric claddings in the onset of EOT resonances.

Regarding experimental results, several single-layer metallic subwavelength hole arrays have been characterized showing EOT resonant peaks in the MIR region. The samples were fabricated with different periods, surrounding claddings and hole dimensions in order to check their influence on the resonance wavelength and quality factor. The measurements show that the resonance wavelength is mainly controlled by the lattice spacing and the dielectric constant of the surrounding claddings. On the other hand, an increase of the hole area causes a transmission enhancement, a slight redshift in the resonances, and a lower quality factor. So, although the simple SPP model relying on grating coupling provides an excellent insight into EOT phenomenon and enables the approximate calculation of transmission peaks, EOT is, in essence, a complex phenomenon where not only the periodicity but also the hole characteristics must be considered when tailoring the structures for a given purpose.

These works were made with the aim of gain insight in the intriguing physics of EOT and negative refraction phenomena in nanostructured metallic films. With the hope that, in turn, the presented breakthroughs will extend the potential of these structures for use in developing modern applications as highly compact optical devices that could become important building blocks in future nano-optical systems, such as ultrafast optical switching or filtering devices. 


\subsection{Future work}

A plenty of room still exists to be further investigated. In the quest of isotropic effective parameters, important aspects are the investigation of the subwavelength hole arrays metamaterial performance under oblique incidence and experimentally retrieve their dispersion relation. Also it is interesting to experimentally explore the functionality of the negative index and EOT achieved under internal SPP excitation in the far-, mid-, nir-infrared and optical ranges because of the metal response variation with frequency. This study can be extended to multilayer structures, especially to the case when different dielectric thicknesses are employed to achieve broad negative index. Additionally, the investigation of the multiple EOT phenomenon, that appears when light couples to dielectric-guided modes, in the case of stacked fishnet metamaterial could expand their functionality in modern applications.

The electromagnetic control of metamaterials leads to new class of practical devices, like polarizers, polarization rotators, and so on. The capacity of multilayer hole arrays structures to tune the dispersion relation's slope (and thus, the right or left-handed propagation behavior) opens up the possibility to achieve a zero slope regime. The investigation of such condition is of great significance for the achievement of metallic absorbers or slow light propagating devices. Additionally, the great electromagnetic confinement achieved with metamaterials makes them suitable candidates in sensing applications. In this way, new metamaterials topologies will be investigated and optimized to be used as accurate sensors with high sensitivities.

By chirping the subwavelength holes, beam steering can be achieved, an exciting application that should be investigated with the ultimate goal of implement a flat lens. Nowadays, plasmonic metamaterials offer an enormous variety of applications that can be implemented even at Terahertz range. This is of great significance, and represents a great opportunity for metamaterials to bridge the THz-gap. In this way, metamaterials operating at $\mathrm{THz}$ range have been characterized (see Fig.20), although new metamaterials need to be explored and prove experimentally their performance at this range. 

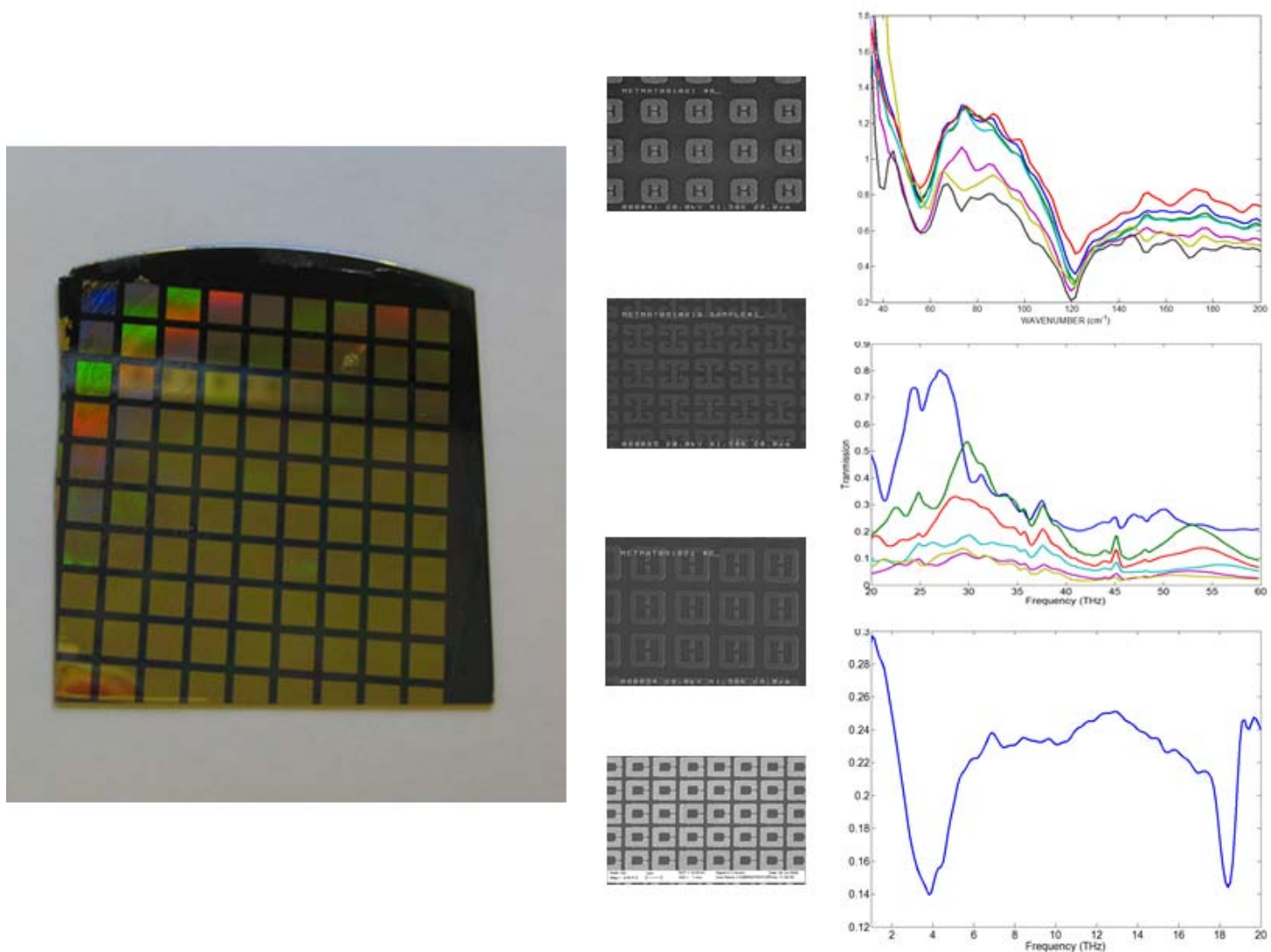

Figure 20. (left) First THz-metamaterial samples over Si wafer fabricated at NTC, (middle) SEM images of some fabricated designs and (right) transmission spectrum measured with FTIR. 


\section{References}

A

[AKA-04] S.S. Akarca-Biyikli, I. Bulu, E. Ozbay, "Enhanced transmission of microwave radiation in one-dimensional metallic gratings with subwavelenght aperture", App. Phys. Lett., vol. 85, pp. 1098-1100, 2004.

[AVR-00] I. Avrutsky, Y. Zhao, V. Kochergin, "Surface-plasmon-assisted resonant tunneling of light through a periodically corrugated thin metal film", Opt. Lett., vol. 25, pp. 595-597, 2000.

B

[BAR-03] W.L. Barnes, A. Dereux, T.W. Ebbesen, "Surface plasmons subwavelength optics", Nature, vol. 424, pp. 824-830, 2003.

[BAR-04] W.L. Barnes, W.A. Murray, J. Dintinger, E. Devaux, T.W. Ebbesen, "Surface plasmons polaritons and their role in the enhanced transmission of light through periodic arrays of subwavelength holes in a metal film", Phys. Rev. Lett., vol. 92, pp. 107401, 2004.

[BER-04] M. Beruete, M. Sorolla, I. Campillo, J.S. Dolado, I. Martín-Moreno, J. Bravo-Abad, F.J. García-Vidal, "Enhanced millimeter-wave transmission through subwavelength hole arrays", Opt. Lett., vol. 29, pp. 2500-2502, 2004.

[BER-05] M. Beruete, M. Sorolla, I. Campillo, J.S. Dolado, L. Martín-Moreno, J. Bravo-Abad, F.J. García-Vidal, "Enhanced millimeter wave transmission through quasioptical subwavelength perforated plates", IEEE Trans. Antenna Propag., vol. 53, pp. 1897-1903, 2005.

[BER-05b] M. Beruete, M. Sorolla, I. Campillo, J.S. Dolado, "Increase of the transmission in cut-off metallic hole arrays", IEEE Microwave and Wirel. Compon. Lett., vol. 15, pp. 116-118, 2005. 
56 REFERENCES

[BER-06] M. Beruete, M. Sorolla, I. Campillo, "Left-handed extraordinary optical transmission through a photonic crystal of subwavelength hole arrays", Opt. Express, vol. 14, pp. 5445, 2006.

[BER-07] M. Beruete, M. Sorolla, M. Navarro-Cía, F. Falcone, I. Campillo, V. Lomakin, "Extraordinary transmission and left-handed propagation in miniaturized stacks of doubly periodic subwavelength hole arrays", Opt. Express, vol. 15, pp. 1107, 2007.

[BET-44] H.A. Bethe, "Theory of diffraction by small holes," Phys. Rev., vol. 66, pp. 163-182, 1944.

[BOA-82] A.D. Boardman, "Electromagnetic Surface Modes", Wiley, New York,1982.

[BOZ-06] S.I. Bozhevolnyi, V.S. Volkov, E. Devaux, J.Y. Laluet, T.W. Ebbesen, "Channel plasmon subwavelength waveguide components including interferometers and ring resonators", Nature, vol. 440, pp. 508-511, 2006.

[BRA-04] J. Bravo-Abad, F.J. García-Vidal, L. Martín-Moreno, "Resonant transmission of light through finite chains of subwavelength holes in a metal film", Phys. Rev. Lett., vol. 93, 227401, 2004.

[BRA-06] J. Bravo-Abad, A. Degiron, F. Przybilla, C. Genet, F.J. García-Vidal, L. Martín-Moreno, T.W. Ebbesen, "How light emerges from an illuminated array of subwavelength holes", Nature Phys., vol. 2, pp. 120-123, 2006.

[BRA-07] J. Bravo-Abad, A.I. Fernández-Domínguez, F.J. García-Vidal, L. MartínMoreno, "Theory of extraordinary transmission of light through quasiperiodic arrays of subwavelength holes", Phys. Rev. Lett., vol. 99, 203905, 2007.

[BRO-99] M.L. Brongersma, J.W. Hartman, H.H. Atwater, "Plasmonics: electromagnetic energy transfer and switching in nanoparticle chain-arrays below the diffraction limit", Molecular Electronics. Symposyum, 1999, Boston, MA, USA. 1999: Warrendale, PA, USA: Mater. Res. Soc., 2001.

C

[CAG-06] H. Caglayan, I. Bulu, E. Ozbay, "Extraordinary grating-coupled microwave transmission through a subwavelength annular aperture", Opt. Express, vol. 13, pp. 1666-1671, 2006.

[CAL-05] C. Caloz, T. Itoh, "Electromagnetic metamaterials: transmission line theory and microwave applications", New Jersey: John Wilet \& Sons, 2005

[CAO-02] Q. Cao, P. Lalanne, "Negative role of surface plasmons in the transmission of metallic gratings with very narrow slits", Phys. Rev. Lett., vol. 88, pp. 7403, 2002. 
[CHE-04] X. Chen, T.M. Grzegorczyk, B.I. Wu, J. Pacheco, J.A.Kong, "Robust method to retrieve the constitutive effective parameters of metamaterial", Phys. Rev. E, vol. 70, 016608, 2004.

[CHA-05] C.W. Chang, A.K. Sarychev, V.M. Shalaev, "Light diffraction by a subwavelength circular aperture", Laser Phys. Lett., vol. 2, pp. 351-355, 2005.

[CHA-06] H.B. Chan, Z. Marcet, K. Woo, D.B. Tanner, D.W. Carr, J.E.Bower, R.A. Cirelli, E. Ferry, F. Klemens, J. Miner, C.S. Pai, J.A. Taylor, "Optical transmission through double-layer metallic subwavelength slit arrays", Opt. Lett., vol. 31, pp. 516-518, 2006.

[CHE-06] H.T. Chen, W.J. Padilla, J.M.O. Zide, A.C. Gossard, A.J. Taylor, R.D. Averitt, "Active terahertz metamaterial devices", Nature, vol. 444, pp. 597600, 2006.

[CHE-07] U.K. Chettiar, A.V. Kildishev, H.K. Yuan, W. Cai, S. Xiao, V.P. Drachev, V.M. Shalaev, "Dual-band negative index metamaterial: double negative at $813 \mathrm{~nm}$ and single negative at $772 \mathrm{~nm}$ ", Opt. Lett., vol. 32, pp. 1671-1673, 2007.

[CUN-74] S.L. Cunningham, A.A. Maradudin, R.F. Wallis, "Effect of a charge layer on the surface-plasmon polariton dispersion curve", Phys. Rev. B, vol. 10, pp. 3342-3355, 1974.

\section{$\mathrm{D}$}

[DAN-09] K. M. Dani, Z. Ku, P. C. Upadhya, R. P. Prasankumar, S. R. J. Brueck, and A. J. Taylor, "Subpicosecond optical switching with a negative index metamaterial," Nano Lett., vol. 9, pp. 3565-3569, 2009.

[DEG-02] A. Degiron, H.J. Lezec, W.L. Barnes, T.W. Ebbesen, "Effects of hole depth on enhanced light transmission through subwavelength hole arrays", App. Phys. Lett., vol. 81, pp. 4327-4329, 2002.

[DEG-04] A. Degiron, H.J. Lezec, N. Yamamoto, T.W. Ebbesen, "Optical transmission properties of a single subwavelength aperture in a real metal", Opt. Commun., vol. 239, pp. 61, 2004.

[DEP-04] R.A. Depine, A. Lakhtakia, "A new condition to identify isotropic dielectric-magnetic materials displaying negative phase velocity", Microw. Opt. Technol. Lett., vol. 41, pp. 315-317, 2004.

[DOL-06] G. Dolling, C. Enkrich, M. Wegener, C.M. Soukoulis, S. Linden, "Low-loss negative-index metamaterial at telecommunication wavelengths", Opt. Lett., vol. 31, pp. 1800-1802, 2006. 
58 REFERENCES

[DOL-06b] G. Dolling, C. Enkrich, M. Wegener, C.M. Soukoulis, S. Linden, "Simultaneous negative phase and group velocity of light in a metamaterial", Science, vol. 312, pp. 892-894, 2006.

[DOL-07] G. Dolling, M. Wegener, C.M. Soukoulis, S. Linden, "Negative-index metamaterial at 780nm wavelength", Opt. Lett., vol. 32, pp. 53-55, 2007.

[DOL-07b] G. Dolling, M. Wegener, S. Linden, "Realization of a three-functional-layer photonic metamaterial", Opt. Lett., vol. 32, pp. 551-553, 2007.

[DRA-85] R. Dragila, B. Luther-Davies, S. Vukovic, "High transparency of classically opaque metallic films," Phys. Rev. Lett., vol. 55, pp. 1117-1120, 1985.

$\mathbf{E}$

[EBB-98] T.W. Ebbesen, H.J. Lezec, H.F. Ghaemi, T. Thio, P.A. Wolff, "Extraordinary optical transmission through sub-wavelength hole arrays", Nature, vol. 391, pp. 667-669, 1998.

[ECO-69] E. N. Economou, "Surface plasmon in thin films", Phys. Rev., vol. 182, pp. 539-554, 1969.

[ELE-06] G.V. Eleftheriades, K.G. Balmain, "Negative-refraction metamaterials: fundamental principles and applications", New Jersey: John Wiley \& Sons, 2005.

$\mathbf{F}$

[FAN-56] U. Fano, "Atomic Theory of electromagnetic interactions in dense materials", Phys. Rev., vol. 103, pp. 1202-1218, 1956.

G

[GAR-02] F.J. García de Abajo, "Light transmission through a single cylindrical hole in a metallic film," Opt. Express, vol. 10, pp. 1475-1484, 2002.

[GAR-03] F.J. García-Vidal, H.J. Lezec, T.W. Ebbesen, L. Martín-Moreno, "Multiple paths to enhance optical transmission through a single subwavelength slit", Phys. Rev. Lett., vol. 90, 213901, 2003.

[GAR-03b] F.J. García-Vidal, L. Martín-Moreno, H.J. Lezec, T.W. Ebbesen, "Focusing light with a single subwavelength aperture flanked by surface corrugations", Appl. Phys. Lett., vol. 83, pp. 4500-4502, 2003.

[GAR-05] F.J. García de Abajo, R. Gómez-Medina, J.J. Sáenz, "Full transmission through perfect-conductor subwavelength hole arrays", Phys. Rev. E, vol. $72,016608,2005$. 
[GAR-05b] F.J. García-Vidal, E. Moreno, J.A. Porto, L. Matín-Moreno, "Transmission of light through a single rectangular hole," Phys. Rev. Lett., vol. 95, 103901, 2005.

[GAR-06] F.J. García de Abajo, J.J. Sáenz, I. Campillo, J.S. Dolado, "Site and lattice resonances in metallic hole arrays", Opt. Express, vol. 7, pp. 7-18, 2006.

[GAR-06b] F.J. García-Vidal, L. Martín-Moreno, Esteban Moreno, L.K. Kumar, R. Gordon, "Transmission of light through a single rectangular hole in a real metal," Phys. Rev. B, vol. 74, pp.153411, 2006.

[GAR-09] C. García-Meca, R. Ortuño, F.J. Rodríguez-Fortuño, J. Martí, A. Martínez, "Negative refraction index metamaterials aided by extraordinary optical transmission", Opt. Express, vol. 17, pp. 6026-6031, 2009.

[GAR-09b] C. García-Meca, R. Ortuño, F.J. Rodríguez-Fortuño, J. Martí, A. Martínez, "Double-negative polarization-independent fishnet metamaterial in the visible spectrum", Opt. Lett., vol. 34, pp. 1603-1605, 2009.

[GAR-11] C. García-Meca, J. Hurtado, J. Martí, A. Martínez, W. Dickson, A.V. Zayats "Low-loss multilayered metamaterial exhibiting a negative index of refraction at visible wavelenghts", Phys. Rev. Lett., vol. 106, 067402, 2011.

[GEN-07] C. Genet, T.W. Ebbesen, "Light in tiny holes", Nature, vol. 445, pp. 39-46, 2007.

[GHA-98] H.F. Ghaemi, T. Thio, D.E. Grupp, T.W. Ebbesen, H.J. Lezec, "Surface plasmons enhance optical transmission through subwavelength holes," Phys. Rev. B, vol. 58, pp. 6779, 1998.

[GRU-00] D.E. Grupp, H.J. Lezec, T.W. Ebbesen, K.M. Pellerin, T. Thio, "Crucial role of metal surface in enhanced transmission through subwavelength apertures," Appl. Phys. Lett., vol. 77, pp. 1569-1571, 2000.

$\mathbf{H}$

[HIB-04] A.P. Hibbins, J.R. Sambles, C.R. Lawrence, J.R. Brown, "Squeezing millimeter waves into microns", Phys. Rev. Lett., vol. 92, 143904, 2004.

[HIB-05] A.P. Hibbins, B.R. Evans, J.R. Sambles, "Experimental verification of designer surface plasmons", Science, vol. 308, pp. 670-672, 2005.

[HOL-03] C.L. Holloway, E.F. Kuester, J. Baker-Jarvis, P. Kabos, "A double negative (DNG) composite medium composed of magnetodielectric spherical particles embedded in a matrix", IEEE Trans. Antennas Propagation, vol. 51, pp. 2596-2603, 2003.

[HUA-06] Z. Huang, J. Xue, Y. Hou, J. Chu, D.H. Zhang, "Optical magnetic response from parallel plate metamaterials", Phys. Rev. B, vol. 74, pp. 193105, 2006. 
$\mathbf{K}$

[KAT-05] N. Katsarakis, G. Konstantinidis, A. Kostopoulos, R.S. Penciu, T.F. Gundogdu, M. Kafesaki, E.N. Economou, T. Koschny, C.M. Soukoulis, "Magnetic response of split-ring resonators in the far-infrared frequency regime", Opt. Lett., vol. 30, pp. 1348-1350, 2005.

[KIM-99] T.J. Kim, T. Thio, T.W. Ebbesen, D.E. Grupp, H.J. Lezec, "Control of optical transmission through metals perforated with subwavelength hole arrays", Opt. Lett., vol. 24, pp. 256, 1999.

[KOE-04] K.J. Koerkamp, S. Enoch, F.B. Segerink, N.F. van Hulst, L. Kuipers, "Strong influence of hole shape on extraordinary transmission through periodic arrays of subwavelength holes," Phys. Rev. Lett., vol. 92, pp. 183901, 2004.

[KOS-03] T. Koschny, P. Markoš, D.R. Smith, C.M. Soukoulis, "Resonant and antiresonant frequency dependence of the effective parameters of metamaterials", Phys. Rev. E, vol. 68, 065602, 2003.

[KOS-05] T. Koschny, L. Zhang, C.M. Soukoulis, "Isotropic three-dimensional lefthanded metamaterials", Phys. Rev. B, vol. 71, 121103, 2005.

[KOS-05b] T. Koschny, P. Markoš, E.N. Economou, D.R. Smith, D.C. Vier, C.M. Soukoulis, "Impact of inherent periodic structure on effective medium description of left-handed and related metamaterials", Phys. Rev. B, vol. 71, 245105, 2005

[KOV-77] G. J. Kovacs, G. D. Scott, "Optical excitation of surface plasma waves in layered media", Phys. Rev. B, vol. 16, pp. 1297-1311, 1977.

[KRA-04] A.V. Krasavin, N.I. Zheludev, "Active plasmonics: Controlling signals in $\mathrm{Au} / \mathrm{Ga}$ waveguide using nanoscale structural transformations", Appl. Phys. Lett., vol. 84, pp. 1416-1418, 2004.

[KRA-05] A.V. Krasavin, A.V. Zayats, N.I. Zheludev, "Active control of surface plamon-polariton waves", J. Opt. A: Pure Appl. Opt., vol. 7, pp. S85-S89, 2005.

[KRE-04] J.R. Krenn, J.C. Weeber, "Surface plasmons polaritons in metal stripes and wires", Phil. Trans. R. Soc. Lond. A, vol. 362, pp.739-756, 2004.

[KRE-68] E. Kretschmann, H. Raether, "Radiative decay of non-radiative surface plasmons excited by light", Z. Naturf., vol. 23A, pp. 2135-2316, 1968.

[KRI-01] A. Krishnan, T. Thio, T.J. Kim, H.J. Lezec, T.W. Ebbesen, P.A. Wolff, J.B. Pendry, L. Matín-Moreno, F.J. García-Vidal, "Evanescently coupled resonance in surface plasmon enhanced transmission", Opt. Comm., vol. 200, 1, 2001.

[KRI-10] C.E. Kriegler, M.S. Rill, S. Linden, M. Wegener, "Bianisotropic photonic metamaterials", IEEE J. Sel. Top. Quant., vol. 16, pp. 367-375, 2010.

[KU-09] Z.Y. Ku, J.Y. Zhang, S.R.J. Brueck, "Bi-anisotropy of multiple-layer fishnet negative-index metamaterials due to angled sidewalls", Opt. Express, vol. 17, pp. 6782-6789, 2009. 
[LAM-04] H. Lamb, "On group-velocity", Pro. Lond. Math. Soc., vol. 1, pp. 473-479, 1904.

[LEZ-02] H.J. Lezec, A. Degiron, E. Deveaux, R.A. Linke, L. Martín-Moreno, F.J. García-Vidal, T.W. Ebbesen, "Beaming light from a sub-wavelength aperture", Science, vol. 297, pp. 820-822, 2002.

[LEZ-04] H.J. Lezec, T. Thio, "Diffracted evanescent wave model for enhanced and suppressed optical transmission through subwavelength hole arrays", Opt. Express, vol. 12, pp. 3629-3651, 2004.

[LI-06] T. Li, H. Liu, F.M. Wang, Z.G. Dong, S.N. Zhu, X. Zhang, "Coupling effect of magnetic polariton in perforated metal/dielectric layered metamaterials and its influence on negative refraction transmission", Opt. Express, vol. 14, pp. 11155-11163, 2006.

[LI-07] T. Li, J.Q. Li, F.M. Wang, Q.J. Wang, H. Liu, S.N. Zhu, Y.Y. Zhu, "Exploring magnetic plasmon polaritons in optical transmission through hole arrays perforated in trilayer structures", Appl. Phys. Lett., vol. 90, 251112, 2007.

[LI-07b] T. Li, H. Liu, F.M. Wang, J.Q. Li, Y.Y. Zhu, S.N. Zhu, "Surface-plasmoninduced optical magnetic response in perforated trilayer metamaterial", Phys. Rev. E, vol. 76, 016606, 2007.

[LI-08] H. Li, S. Xie, R. Zhou, Q. Liu, X. Zhou, M. Yuan, "Two different transmission tunnels of light through double-layer gold nanohole arrays", J. Phys.: Condens. Matter, vol. 20, 415223, 2008.

[LIU-07] N. Liu, H. Go, L. Fu, S. Kaiser, H. Schweizer, H. Giessen, "Plasmon Hybridization in Stacked Cut-Wire Metamaterials", Adv. Mater., vol. 19, pp. 3628-3632, 2007.

[LIU-08] N. Liu, H. Guo, L. Fu, S. Kaiser, H. Schweizer, H. Giessen, "Threedimensional photonic metamaterials at optical frequencies", Nature Mater., vol. 7, pp. 31-37, 2008.

[LIU-08b] N. Liu, L. Fu, S. Kaiser, H. Schweizer, H. Giessen, "Plasmonic building blocks for magnetic molecules in three-dimensional optical metamaterials", Adv. Mater., vol. 20, pp. 3859-3865, 2008.

[LOM-04] V. Lomakin, N.W. Chen, S.Q. Li, E. Michielssen, "Enhanced transmission through two-period arrays of sub-wavelength holes", IEEE Microw. Wireless Compon. Lett., vol. 14, pp. 355-357, 2004.

[LOM-05] V. Lomakin, E. Michielssen, "Enhanced transmission through metallic plates perforated by arrays of subwavelength holes sandwiched between dielectric slabs", Phys. Rev. B, vol. 71, 235117, 2005. 
[MAI-03] S.A. Maier, P.G. Kik, H.A. Atwater, S. Meltzer, E. Harel, B.E. Koel, A.A.G. Requicha, "Local detection of electromagnetic energy transport below the diffraction limit in metal nanoparticle plasmon waveguides", Nature Mat., vol. 2, pp. 229-232, 2003.

[MAI-07] S.A. Maier, "Plasmonics: Fundamental and Applications", Springer, New York, 2007.

[MAN-45] L.I. Mandel'shtam, "Group velocity in crystalline arrays", Zh. Eksp. Teor. Fiz., vol. 15, pp. 475-478, 1945.

[MAR-01] L. Martín-Moreno, F.J. García-Vidal, H.J. Lezec, K.M. Pellerin, T.Thio, D.E. Grupp, J.B. Pendry, T.W. Ebbesen, "Theory of extraordinary optical transmission trough subwavelength hole arrays," Phys. Rev. Lett., vol. 86, pp. 1114, 2001.

[MAR-03] L. Martín-Moreno, F.J. García-Vidal, H.J. Lezec, A. Degiron, T.W. Ebbesen, "Theory of highly directional emission from a single subwavelength aperture surrounded by surface corrugations", Phys. Rev. Lett., vol. 90, 167401, 2003.

[MAR-03b] P. Markoš, C.M. Soukoulis, "Transmission properties and effective electromagnetic parameters of double negative metamaterials", Opt. Express, vol. 11, pp. 649-661, 2003.

[MAR-05] A. Martínez, J. Martí, "Negative refraction in two-dimensional photonic crystals: role of the lattice orientation and the interface termination," Phys. Rev. B, vol. 71, pp. 235115, 2005.

[MAR-08] A. Mary, S.G. Rodrigo, F.J. García-Vidal, L. Martín-Moreno, "Theory of negative-refractive-index response of double-fishnet structures", Phys. Rev. Lett., vol. 101, pp. 103902, 2008.

[MAT-07] T. Matsui, A. Agrawal, A. Nahata, Z.V. Vardeny, "Transmission resonances through aperiodic arrays of subwavelength apertures", Nature, vol. 446, pp. 517-521, 2007.

[MIY-05] F. Miyamaru, M. Hangyo, "Anomalous terahertz transmission through double-layer metal hole arrays by coupling of surface plasmon polaritons", Phys. Rev. B, vol. 71, 165408, 2005.

[MIY-10] F. Miyamaru, S. Kuboda, K. Taima, K. Takano, M. Hangyo, M.W. Takeda, "Three-dimensional bulk metamaterials operating in the terahertz range", App. Phys. Lett., vol. 96, pp. 081105, 2010.

[MUN-00] B.A. Munk, "Frequency selective surfaces: theory and design", Wiley Interscience, New York, 2000. 
[NAV-08] M. Navarro-Cía, M. Beruete, M. Sorolla, I. Campillo, "Negative refraction in a prism made of stacked subwavelength hole arrays", Opt. Express, vol. 16, pp. 560, 2008.

[NOR-04] P. Nordlander, C. Oubre, E. Prodan, K. Li, M.I. Stockman, "Plasmon Hybridization in Nanoparticle Dimers" Nano Lett., vol. 4, pp. 899-903, 2004.

[NOV-02] I.V. Novikov, A.A. Maradudin, "Channel polaritons", Phys. Rev. B, vol. 66, 035403, 2002.

[OTT-68] A. Otto, "Excitation of nonradiative surface plasma waves in silver by the method of frustrated total reflection", Z. Phys., vol. 216, pp. 398-410, 1968.

[OZB-06] E. Ozbay, "Plasmonics: merging photonics and electronics at nanoscale dimensions", Science, vol. 311, pp. 189-193, 2006.

$\mathbf{P}$

[PAU-09] O. Paul, C. Imhof, B. Lägel, S. Wolff, J. Heinrich, S. Höfling, A. Forchel, R. Zengerle, R. Beigang, M. Rahm, "Polarization-independent active metamaterial for high-frequency terahertz modulation", Opt. Express, vol. 17, pp. 819-827, 2009.

[PEN-96] J.B. Pendry, A.J. Holden, W.J. Stewart, I. Youngs, "Extremely low frequency plasmons in metallic mesostructures", Phys. Rev. Lett., vol. 76, pp. 4773-4776, 1996.

[PEN-98] J.B. Pendry, A.J. Holden, D.J. Robbins, W.J. Stewart, "Low frequency plasmons in thin-wire structures", J. Physics: Condensed Matt., vol. 10, pp. 4785-4809, 1998.

[PEN-99] J.B. Pendry, "Playing tricks with light", Science, vol. 285, pp. 1687-1688, 1999.

[PEN-99b] J.B. Pendry, A.J. Holden, D.J. Robbins, W.J. Stewart, "Magnetism from conductors and enhanced nonlinear phenomena", IEEE Trans. Microw. Theory Tech., vol. 47, pp. 2075-2084, 1999.

[PEN-00] J.B. Pendry, "Negative refractions makes a perfect lens", Phys. Rev. Lett., vol. 85, pp. 3966-3969, 2000.

[PEN-04] J.B. Pendry, L. Martín-Moreno, F.J. García-Vidal, "Mimicking surface plasmons with structured surfaces", Science, vol. 305, pp. 847-848, 2004.

[PIN-56] D. Pines, "Collective energy losses in solids", Rev. Mod. Phys., vol. 28, pp. 184-198, 1956. 
64 REFERENCES

[POD-05] V.A. Podolsky, E.E. Narimanov, "Near-sighted superlens", Opt. Lett., vol. 30, pp. 75-77, 2005.

[POP-00] E. Popov, M. Neviere, S. Enoch, R. Reinisch, "Theory of light transmission through subwavelength periodic hole arrays", Phys. Rev. B, vol. 62, pp. 16100-16108, 2000.

[POP-05] E. Popov, N. Bonod, M. Nevière, H. Rigneault, P.F. Lenne, P. Chaumet, "Surface plasmon excitation on a single subwavelength hole in a metallic sheet", Appl. Opt., vol. 44, pp. 2332-2337, 2005.

[POR-99] J.A. Porto, F.J. García-Vidal, J.B. Pendry, "Transmission resonances on metallic gratings with very narrow slits", Phys. Rev. Lett., vol. 83, pp. 2845-2848, 1999.

[PRO-03] E. Prodan, C. Radloff, N.J. Halas, P. Nordlander, "A hybridization model for the plasmon response of complex nanostructures", Science, vol. 302, pp. 419-422, 2003.

[PRZ-06] F. Przybilla, C. Genet, T.W. Ebbesen, "Enhanced transmission through Penrose subwavelength hole arrays", Appl. Phys. Lett., vol. 89, 121115, 2006.

$\mathbf{R}$

[RAE-86] H. Raether, "Surface plasmons on smooth and rough surfaces and on gratings", Ed. Springer-Verlag, New York, 1986.

[RIT-57] R.H. Ritchie, "Plasma losses by fast electrons in thin films", Phys. Rev., vol. 106, pp. 874-881, 1957.

[RIT-68] R.H. Ritchie, E.T. Arakawa, J.J. Cowan, R.N. Hamm, "Surface-plasmon resonance effect in grating diffraction", Phys. Rev. Lett., vol. 21, pp. 1530$1532,1968$.

[ROB-87] A. Roberts, "Electromagnetic theory of diffraction by a circular aperture in a thick, perfectly conducting screen", J. Opt. Soc. Am. A, vol. 4, pp. 19701983, 1987.

[RUA-06] Z. Ruan, M. Qiu, "Enhanced transmission through periodic arrays of subwavelength holes: the role of localized waveguide resonances," Phys. Rev. Lett., vol. 96, pp. 233901, 2006.

$\mathrm{S}$

[SAR-03] M. Sarrazin, J.P. Vigneron, J.M. Vigoureux, "Role of Wood anomalies in optical properties of thin metallic films with a bidimensional array of subwavelength holes", Phys. Rev. B, vol. 67, 085415, 2003.

[SAR-05] M. Sarrazin, J.P. Vigneron, "Light transmission assisted by BrewsterZenneck modes in chromium films carrying a subwavelength hole array", Phys. Rev. B, vol. 71, 075404, 2005. 
[SCH-04] A. Schuster, "An introduction to the theory of optics", Ed. Edward Arnold, London, U.K., 1904.

[SHA-05] V.M. Shalaev, W. Cai, U.K. Chettiar, H.K. Yuan, A.K. Sarychev, V.P. Drachev, A.V. Kildishev, "Negative index of refraction in optical metamaterials", Opt. Lett., vol. 30, pp. 3356-3358, 2005.

[SHE-01] R.A. Shelby, D.R. Smith, S.C. Nemat-Nasser, S. Schultz, "Microwave transmission through a two-dimensional, isotropic, left-handed material", Appl. Phys. Lett., vol. 78, pp. 489-491, 2001.

[SHE-01b] R.A. Shelby, D.R. Smith, S. Schultz, "Experimental verification of a negative index of refraction" Science, vol. 292, pp. 77-79, 2001.

[SHR-11] D. Shrekenhamer, S. Rout, A.C. Strikwerda, C. Bingham, R.D. Averitt, S. Sonkusale, W.J. Padilla, "High speed terahertz modulation from metamaterials with embedded high electron mobility transistors", Opt. Express, vol. 19, pp. 9968-9975, 2011.

[SMI-00] D.R. Smith, W.J. Padilla, D.C. Vier, S.C. Nemat-Nasser, S. Schultz, "Composite medium with simultaneously negative permeability and permittivity", Phys. Rev. Lett., vol. 84, pp. 4184-4187, 2000.

[SMI-02] D.R. Smith, S. Schultz, P. Markos, C.M. Soukoulis, "Determination of effective permittivity and permeability of metamaterials from reflection and transmission coefficients", Phys. Rev. B, vol. 65, pp. 195104, 2002.

[SMI-03] D.R. Smith, D. Schurig, M. Rosenbluth, S. Schultz, S.A. Ramakrishna, J.B. Pendry, "Limitations on subdiffraction imaging with a negative refractive index slab", App. Phys. Lett., vol. 82, pp. 1506-1508, 2003.

[SMI-05] D.R. Smith, D.C. Vier, T. Koschny, C.M. Soukoulis, "Electromagnetic parameter retrieval from inhomogeneous metamaterials", Phys. Rev. E, vol. 71, pp. 036617, 2005.

[SOM-99] A. Sommerfeld, "Über die Fortpflanzung electrodynamischer Wellen längs eines Drahtes", Ann. Phys. und Chemie, vol. 67, pp. 233-290, 1899.

[SOU-10] C.M. Soukoulis, M. Wegener, "Optical metamaterials: more bulky and less lossy", Science, vol. 330, pp. 1633-1634, 2010.

[SOu-11] C.M. soukoulis, M. Wegener, "Past achievements and future challenges in the development of three-dimensional photonic metamaterials", Nature Phot., vol. 5, pp. 523-530, 2011.

$\mathrm{T}$

[TAK-01] Y. Takakura, "Optical resonance in a narrow slit in a thick metallic screen," Phys. Rev. Lett., vol. 86, pp. 5601-5603, 2001.

[TAN-07] Z.H. Tang, R.W. Peng, Z. Wang, X. Wu, Y.J. Bao, Q.J. Wang, Z.J. Zhang, W.H. Sun, M. Wang, "Coupling of surface plasmons in nanostructured metal/dielectric multilayers with subwavelength hole arrays", Phys. Rev. B, vol. 76, 195405, 2007. 
66 REFERENCES

[THI-99] T. Thio, H.F. Ghaemi, H.J. Lezec, P.A. Wolff, T.W. Ebbesen, "Surfaceplasmon-enhanced transmission through hole arrays in Cr films", J. Opt. Soc. Am. B, vol. 16, pp.1743-1748, 1999.

[THI-01] T. Thio, K.M. Pellerin, R.A. Linke, H.J. Lezec, T.W. Ebbesen, "Enhanced light transmission through a single subwavelength aperture", Opt. Lett., vol. 26, pp. 1972-1974, 2001.

[TRE-99] M.M.J. Treacy, "Dynamical diffraction in metallic optical gratings", Appl. Phys. Lett., vol. 75, pp. 606-608, 1999.

[TRE-02] M.M.J. Treacy, "Dynamical diffraction explanation of the anomalous transmission of light through metallic gratings", Phys. Rev. B, vol. 66, 195105, 2002.

V

[VAL-08] J. Valentine, S. Zhang, T. Zentgraf, E. Ulin-Avila, D.A. Genov, G. Bartal, $\mathrm{X}$. Zhang, "Three-dimensional optical metamaterial with a negative refractive index", Nature, vol. 455, pp. 376-379, 2008.

[VES-68] V.G. Veselago, "The electrodynamics of substances with simultaneously negative values of $\varepsilon$ and $\mu$ ", Soviet Physics Uspekhi, vol. 10, pp. 509-514, 1968.

W

[WAN-03] Y. Wang, "Wavelength selection with coupled surface plasmon waves", Appl. Phys. Lett., vol. 82, pp. 4385-4387, 2003.

[WAN-06] H. Wang, D.W. Brandi, F. Le, P. Nordlander, N J. Halas, "Nanorice: A Hybrid Plasmonic Nanostructure", Nano Lett., vol. 6, pp. 827-832, 2006.

[WEB-04] K.J. Webb, M. Yang, D.W. Ward, K.A. Nelson, "Metrics for negativerefractive-index materials", Phys. Rev. E, vol. 70, 035602, 2004.

[WEB-06] K.J. Webb, J. Li, " Analysis of transmission through small apertures in conducting films", Phys. Rev. B, vol. 73, 033401, 2006.

[WOO-02] R.W. Wood, "On a remarkable case of uneven distribution of light in a diffraction grating spectrum", Phil. Mag., vol. 4, pp. 396-402, 1902.

[WOO-35] R.W. Wood, "Anomalous diffraction gratings", Phys. Rev., vol. 48, pp. 928-936, 1935.

$\mathrm{X}$

[XIA-09] S. Xiao, U.K. Chettiar, A.V. Kildishev, V.P. Drachev, V.M. Shalaev, "Yellow-light negative-index metamaterials", Opt. Lett., vol. 34, pp. 34783480, 2009. 
[XIA-10] S. Xiao, V.P. Drachev, A.V. Kildishev, X. Ni, U.K. Chettiar, H.K. Yuan, V.M. Shalaev, "Loss-free and active optical negative-index metamaterials", Nature, vol. 466, pp. 735-738, 2010.

Y

[YE-05] Y.H. Ye, Y. Zhang, "Enhanced light transmission through cascaded metal films perforated with periodic hole arrays", Opt. Lett., vol. 30, pp. 15211523, 2005.

[YIN-04] L. Yin, V.K. Vlasko-Vlasov, A. Rydh, J. Pearson, U. Welp, S.H. Chang, S.K. Gray, G.C. Schatz, D.B. Brown, C.W. Kimball, "Surface plasmons at single nanoholes in Au films", Appl. Phys. Lett., vol. 85, pp. 467-469, 2004.

\section{$\mathrm{Z}$}

[ZEN-07] J. Zenneck, "Über die Fortpflanzung ebener elektromagnetischer Wellen längs einer ebenen Leiterfläche und ihre Beziehung zur drahtlosen Telegraphie", Ann. d. Phys., vol. 23, pp. 846-866, 1907.

[ZHA-05] S. Zhang, W. Fan, N.C. Panoiu, K.J. Malloy, R.M. Osgood, S.R.J. Brueck, "Experimental demonstration of near-infrared negative-index metamaterials", Phys. Rev. Lett., vol. 95, 137404, 2005.

[ZHA-05b] S. Zhang, W. Fan, K.J. Malloy, S.R.J. Brueck, N.C. Panoiu, R.M. Osgood, "Near-infrared double negative metamaterials", Opt. Express, vol. 13, pp. 4922-4930, 2005.

[ZHO-05] L. Zhou, W. Wen, C.T. Chan, P. Sheng, "Electromagnetic-wave tunnelling through negative-permittivity media with high magnetic fields", Phys. Rev. Lett., vol. 94, pp. 243905, 2005. 



\section{Author's merits}

\section{Journal Papers}

R. Ortuño, C. García-Meca, A. Martínez, "Role of the lens thickness and the surface termination in the formation of subwavelength images by a negative-index photonic-crystal slab", The Open Opt. Journal, vol. 2, pp. 79-85, 2008.

[2] R. Ortuño, C. García-Meca, F.J. Rodríguez-Fortuño, J. Martí, A. Martínez, "Role of surface plasmon polaritons on optical transmission through double layer metallic hole arrays", Phys. Rev. B, vol. 79, pp. $75425,2009$.

[3] R. Ortuño, C. García-Meca, F.J. Rodríguez-Fortuño, A. Martínez, "Enlarging the negative-index bandwidth of optical metamaterials by hybridized plasmon resonances", Opt. Lett., vol. 35, pp. 4205-4207, 2010.

[4] R. Ortuño, C. García-Meca, F.J. Rodríguez-Fortuño, J. Martí, A. Martínez, "Multiple extraordinary optical transmission peaks from evanescent coupling in perforated metal plates surrounded by dielectrics", Opt. Express, vol. 18, pp. 7893-7898, 2010.

[5] R. Ortuño, C. García-Meca, F.J. Rodríguez-Fortuño, A. Hakansson, A. Griol, J. Hurtado, J.A. Ayúcar, L. Bellieres, P.J. Rodríguez, F. LópezRoyo, J. Martí, A. Martínez, "Midinfrared filters based on extraordinary optical transmission through subwavelength structured gold films", J. App. Opt., vol. 106, pp. 124313, 2009.

[6] F.J. Rodríguez-Fortuño, C. García-Meca, R. Ortuño, J. Martí, A. Martínez, "Coaxial plasmonic waveguide array as a negative-index metamaterial", Opt. Lett., vol. 34, pp. 3325- 3327, 2009.

[7] C. García-Meca, R. Ortuño, F.J. Rodríguez-Fortuño, J. Martí, A. Martínez, "Double-negative polarization-independent fishnet metamaterial in the visible spectrum", Opt. Lett., vol. 34, pp. 1603-1605, 2009.

[8] C. García-Meca, R. Ortuño, R. Salvador, A. Martínez, J. Martí, "Lowloss single-layer metamaterial with negative index of refraction at visible wavelengths", Opt. Express., vol. 15, pp. 9320-9325, 2007.

[9] A. Mártínez, C. García-Meca, R. Ortuño, F.J. Rodríguez-Fortuño, J. 
Martí, "Metamaterials for optical security", App. Phys. Lett., vol. 94, pp. 251106- 251108, 2009.

[10] F.J. Rodríguez-Fortuño, C. García-Meca, R. Ortuño, A. Martínez, J. Martí, "Modeling high-order plasmon resonances of a U-shaped nanowire used to build a negative-index metamaterial", Phys. Rev. B, vol. 79, pp. 75103, 2009.

[11] C. García-Meca, R. Ortuño, F.J. Rodríguez-Fortuño, J. Martí, A. Martínez, "Negative refractive index metamaterials aided by extraordinary optical transmission", Opt. Express, vol. 17, pp. 6026-6031, 2009.

[12] F.J. Rodríguez-Fortuño, C. García-Meca, R. Ortuño, J. Martí, A. Martínez, "Zero-bandwidth mode in a split-ring-resonator-loaded onedimensional photonic crystal", Phys. Rev. B, vol. 81, pp. 233101, 2010.

[13] C. García-Meca, M.M. Tung, J.V. Galán, R. Ortuño, F.J. RodríguezFortuño, J. Martí, A. Martínez, "Squeezing and expanding light without reflections via transformation optics", Optics Express, vol. 19, pp.35623575, 2011.

[14] P.J. Rodríguez-Cantó, M. Martínez-Marco, F.J. Rodríguez-Fortuño, B. Tomás-Navarro, R. Ortuño, S. Peransí-Llopis, A. Martínez, "Demonstration of near infrared gas sensing using gold nanodisks on functionalized silicon" Optics Express, vol. 19, pp.7664-7672,2011.

[15] F.J. Rodríguez-Fortuño, M. Martínez-Marco, B. Tomás-Navarro, R. Ortuño, J. Martí, A. Martínez, P.J. Rodríguez-Cantó, "Highly-sensitive chemical detection in the infrared regime using plasmonic gold nanocrosses", Appl. Phys. Lett. 98, 133118, 2011.

[16] R. Salvador, A. Martínez, C. García-Meca, R. Ortuño, J. Martí, "Analysis of hybrid dielectric-plasmonic waveguides", J. Sel. Topics Quant. Electr., vol. 14, pp. 1496-1501, 2008.

\section{Conferences}

[14] A. Martínez, R. Ortuño, J. Martí, "Subwavelength imaging by a negative-index photonic-crystal slab: role of the thickness and surface cut", Adv. Elect. Mat. in Microwaves and Optics - Metamaterials, Rome, 2007.

[15] A. Martínez, R. Ortuño, J. Martí, "Influence of the thickness on the subwavelength imaging by a negative-index photonic-crystal lens", Young Scientist Meeting on Metamaterials, Seville, 2006.

[16] R. Ortuño, C. García-Meca, F.J. Rodríguez-Fortuño, J. Martí, A. Martínez, "Effect of internal and external surface plasmons in the enhanced transmission through double-layer metallic hole arrays", Adv. Elect. Mat. in Microwaves and Optics - Metamaterials, Pamplona, 2008.

[17] R. Ortuño, A. Hakansson, J. Hurtado, L. Bellieres, P.J. Rodríguez, A. Griol, J.A. Ayúcar, F. López-Royo, C. García-Meca, F.J. RodríguezFortuño, J. Martí, A. Martínez, "MIR filters based on extraordinary transmission through nanostructured gold films", Adv. Elect. Mat. in Microwaves and Optics - Metamaterials, London, 2009. 
[18] R. Ortuño, C. García-Meca, F.J. Rodríguez-Fortuño, J. Martí, A. Martínez, "Multiple extraordinary optical transmission peaks from evanescent coupling in perforated metal plates", Adv. Elect. Mat. in Microwaves and Optics - Metamaterials, London, 2009.

[19] R. Ortuño, C. García-Meca, F.J. Rodríguez-Fortuño, J. Martí, A. Martínez, "Enlarged negative effective index bandwith from fishnet metamaterials", SPIE Europe International Symposium Photonics Europe, Brussels, 2010.

[20] R. Ortuño, C. García-Meca, J.F. Rodríguez-Fortuño, J. Martí, A. Martínez, "Extraordinary transmission through metamaterials arrays at $\mathrm{THz}$ frequencies", Young Scientist Meeting on Metamaterials, Valencia, 2011.

[21] R. Ortuño, C. Garcia-Meca, F.J. Rodriguez-Fortuno, A. Martínez, "Extraordinary transmission and light confinement in subwavelength metallic films apertures", Progress In Electromagnetics Research Symposium, Marrakech, 2011.

[22] C. García-Meca, R. Ortuño, F.J. Rodríguez-Fortuño, J. Martí, A. Martínez, "Double-negative polarization-independent fishnet metamaterial operating in the visible", IEEE LEOS Winter Topicals, Innsbruck, 2009.

[23] F.J. Rodríguez-Fortuño, C. García-Meca, R. Ortuño, J. Martí, A. Martínez , "Negative index metamaterial through high-order plasmon resonances on u-shaped nanowires", IEEE LEOS Winter Topicals, Innsbruck, 2009.

[24] A. Ros, C.W. Lerche, F. Sanchez, J. Segura, R. Ortuño, J. Martí; A. Cantarero, A. Sebastia, J.M. Benlloch, "Impact of the scattering coefficient of scintillation crystals (LYSO and LSO) on depth of interaction resolution", IEEE Nuclear Science Symposium and Medical Imaging Conference, Dresden, 2008.

[25] C. García-Meca, R. Ortuño, F.J. Rodríguez-Fortuño, A. Martínez, J. Martí, "Extraordinary optical transmission with negative index of refraction", Adv. Elect. Mat. in Microwaves and Optics - Metamaterials, Pamplona, 2008.

[26] C. García-Meca, R. Ortuño, A. Martínez, J. Martí, "Low-loss single-layer metamaterial with negative index of refraction at visible frequencies", Adv. Elect. Mat. in Microwaves and Optics - Metamaterials, Rome, 2007.

[27] F.J. Rodríguez-Fortuño, R. Ortuño, C. García-Meca, J. Martí, A. Martínez, "Split-ring resonators achieve transmission through a photonic crystal bandgap", Adv. Elect. Mat. in Microwaves and Optics Metamaterials, London, 2009.

[28] M.L. Martínez, P.J. Rodríguez, F.J. Rodríguez-Fortuño, R. Ortuño, A. Martínez, "Plasmonic sensor based on gold nanodisk structures for toxic organic molecules detection", Adv. Elect. Mat. in Microwaves and Optics Metamaterials, Karlsruhe, 2010. 
[29] F.J. Rodríguez-Fortuño, C. García-Meca, R. Ortuño, J. Martí, A. Martínez, "Split-ring-resonators inserted in a photonic crystal achieve a zerobandwidth passband", Adv. Elect. Mat. in Microwaves and Optics Metamaterials, Karlsruhe, 2010.

[30] C. García-Meca, R. Ortuño, F.J. Rodríguez-Fortuño, J. Martí, A. Martínez, "Strong magnetism at visible wavelengths via coupled silver nano-hoops", Adv. Elect. Mat. in Microwaves and Optics - Metamaterials, London, 2009.

[31] C. García-Meca, M. Tung, J.V. Galan, R. Ortuño, F.J. RodríguezFortuño, J. Martí, A. Martínez, "Light compression without reflections", SPIE Europe International Symposium Photonics Europe, Brussels, 2010.

[32] F.J. Rodríguez-Fortuño, R. Ortuño, C. García-Meca, J. Martí, A. Martínez, "Modeling and understanding plasmonic resonances in optical metamaterials", Young Scientist Meeting on Metamaterials, Leganés, 2009.

[33] M. Martínez-Marco, F.J. Rodríguez-Fortuño, P.J. Rodríguez, R. Ortuño, A. Martínez, "Plasmonic sensor based on gold nanodisk structures for toxic organic molecule detection", Conferencia Española de Nanofotónica 2010 (CEN2010), Segovia, 2010.

[34] F.J. Rodríguez-Fortuño, R. Ortuño, C. García-Meca, A. Martínez, "Zero bandwidth mode on a split ring resonator loaded waveguide at cutoff", Conferencia Española de Nanofotónica 2010 (CEN2010), Segovia, 2010.

[35] C. García-Meca, R. Ortuño, F.J. Rodríguez-Fortuño, J. Martí, A. Martínez, "Flat and reflectionless solid immersion lens based on transformation optics", Young Scientist Meeting on Metamaterials, Valencia, 2011.

[36] F.J. Rodríguez-Fortuño, M. Martínez-Marco, B. Tomás-Navarro, R. Ortuño, A. Martínez, P. J. Rodríguez-Cantó, "Chemical sensor using the localized surface plasmon resonance of arrayed gold nanocrosses", Young Scientist Meeting on Metamaterials, Valencia, 2011.

[37] M. Lorente-Crespo, F.J. Rodríguez-Fortuño, R. Ortuño, C. García-Meca, A. Martínez, "Magnetic metamaterials in the Visible Range based on nanohoops and nano-squares", Young Scientist Meeting on Metamaterials, Valencia, 2011. 